\title{
Highly modular piano-stool N-heterocyclic carbene iron complexes: impact of ligand variation on hydrosilylation activity
}

\author{
Pamela V. S. Nylund, ${ }^{\dagger}$ Nathalie C. Ségaud ${ }^{\dagger}$ and Martin Albrecht $*, \dagger$ \\ Department of Chemistry \& Biochemistry, University of Bern, Freiestrasse 3, 3012 Bern, \\ Switzerland
}

Email: martin.albrecht@dcb.unibe.ch

\section{Content}

1. Ligand synthesis $\quad$ S2

2. NMR spectra of all new compounds S3

3. Structural analyses by NOE experiments $\quad$ S28

4. Calculations for free energy of activation $\quad$ S30

5. Cyclic voltammetry of neutral NHC iron complexes S31

6. Crystallographic details $\quad \mathrm{S} 32$

7. Steric maps of neutral NHC iron complexes $\quad$ S37

8. Catalytic details $\quad \mathrm{S} 38$

$\begin{array}{ll}\text { 9. Mechanistic experiments } & \text { S39 }\end{array}$

10. References $\quad$ S51 


\section{Ligand synthesis}

Toluene, THF and $\mathrm{Et}_{2} \mathrm{O}$ were dried by passage through solvent purification columns. All other reagents were commercially available and used without further purification. Carboxylation reactions were carried out under an inert nitrogen atmosphere using standard Schlenk techniques. The synthesis of 1,4-isopropyl-1,2,3-triazole, ${ }^{\mathrm{S} 1}$ azolium salts $\mathbf{1 a},{ }^{\mathrm{S} 2} \mathbf{1 b},{ }^{\mathrm{S} 3} \mathbf{1 c},{ }^{\mathrm{S} 4} \mathbf{1 d},{ }^{\mathrm{S} 5} \mathbf{1 f},{ }^{\mathrm{S} 6} \mathbf{1 g},{ }^{\mathrm{S} 6}$ and the imidazolium-2carboxylates $2 \mathbf{a}^{\mathrm{S} 7}$ and $\mathbf{2} \mathbf{b}^{\mathrm{S} 8}$ have been reported elsewhere. NMR spectra were measured at $25{ }^{\circ} \mathrm{C}$ on Bruker spectrometers operating at 300 or $400 \mathrm{MHz}\left({ }^{1} \mathrm{H}\right.$ NMR) and 75 or $101 \mathrm{MHz}\left({ }^{13} \mathrm{C}\left\{{ }^{1} \mathrm{H}\right\} \mathrm{NMR}\right)$, respectively. Chemical shifts ( $\delta$ in ppm, coupling constants $\mathrm{J}$ in $\mathrm{Hz}$ ) were referenced to residual solvent resonances downfield to $\mathrm{SiMe}_{4}$. Assignments were made based on homo- and heteronuclear shift correlation spectroscopy. Elemental analysis was performed at the University of Bern Microanalytic Laboratory by using a Thermo Scientific Flash 2000 CHNS-O elemental analyser. High-resolution mass spectrometry was carried out with a Thermo Scientific LTQ Orbitrap XL instrument (ESI-TOF). IR spectra were recorded on a Jasco 4700 FT-IR instrument in $\mathrm{CH}_{2} \mathrm{Cl}_{2}$ solution at $1 \mathrm{~cm}^{-1}$ resolution.

Synthesis of 1,4-iso-propyl-2-methyl-1,2,3-triazolium iodide (1e): To a solution of 1,4-iso-propyl1,2,3-triazole (720 mg, $4.7 \mathrm{mmol})$ in $\mathrm{MeCN}(14 \mathrm{~mL})$ was added MeI $(0.6 \mathrm{~mL}, 9.4 \mathrm{mmol})$. The mixture was refluxed for $48 \mathrm{~h}$. The volatiles were removed under reduced pressure and the residual orange oil was dissolved in THF and precipitated with $\mathrm{Et}_{2} \mathrm{O}$ to yield an orange solid (1.4 g, 96\%). ${ }^{1} \mathrm{H}$ NMR (400 $\left.\mathrm{MHz}, \mathrm{CDCl}_{3}\right): \delta=9.23\left(\mathrm{~s}, 1 \mathrm{H}, \mathrm{H}_{\mathrm{trz}}\right), 5.25$ (sept, $\left.J_{\mathrm{HH}}=6.7 \mathrm{~Hz}, 1 \mathrm{H}, \mathrm{N}_{\mathrm{trz}} \mathrm{CH}\right), 4.32\left(\mathrm{~s}, 3 \mathrm{H}, \mathrm{NCH}_{3}\right), 3.34$ (sept, $\left.J_{\mathrm{HH}}=6.9 \mathrm{~Hz}, 1 \mathrm{H}, \mathrm{C}_{\mathrm{trz}} \mathrm{CH}\right), 1.71\left(\mathrm{~d}, J=6.9 \mathrm{~Hz}, 6 \mathrm{H}, \mathrm{CH}_{3}\right), 1.45\left(\mathrm{~d}, J=6.7 \mathrm{~Hz}, 6 \mathrm{H}, \mathrm{CH}_{3}\right) .{ }^{13} \mathrm{C}\left\{{ }^{1} \mathrm{H}\right\}$ NMR (75 MHz, $\left.\mathrm{CDCl}_{3}\right): \delta=149.91\left(\mathrm{C}_{\mathrm{trz}}-i \mathrm{Pr}\right), 127.48\left(\mathrm{C}_{\mathrm{trz}} \mathrm{H}\right), 58.55(\mathrm{NCH}), 38.90\left(\mathrm{NCH}_{3}\right), 24.94$ $\left(\mathrm{C}_{\text {trz }} \mathrm{CH}\right), 22.59,21.56\left(2 \times \mathrm{CH}-\mathrm{CH}_{3}\right)$. HRMS: m/z: calcd. for $\mathrm{C}_{9} \mathrm{H}_{18} \mathrm{~N}_{3}[\mathrm{M}-\mathrm{I}]^{+}$: 168.1495; found: 168.1495. Elemental Analysis calcd. (\%) for $\mathrm{C}_{9} \mathrm{H}_{18} \mathrm{IN}_{3}$ (295.17): C 36.62, H 6.15, N 14.24; found: C 36.22, H 5.70, N 13.81 .

Synthesis of triazolium carboxylate (2f): The triazolium salt $\mathbf{1 f}(516 \mathrm{mg}, 1.33 \mathrm{mmol})$ and $\mathrm{KOtBu}(370$ $\mathrm{mg}, 3.33 \mathrm{mmol})$ were suspended in dry THF $(10 \mathrm{~mL})$. After $20 \mathrm{~min}$ of stirring at room temperature, the THF was removed under reduced pressure and the free carbene extracted in dry toluene $(6 \mathrm{~mL} \times 2)$ the resulting suspension was filtered into an oven-dried schlenk equipped with a magnetic stir bar, the solution was bubbled with $\mathrm{CO}_{2}$ for $1 \mathrm{~h}$ leading to the formation of a white precipitate. The precipitate was collected and subsequent washing with $\mathrm{Et}_{2} \mathrm{O}$ afforded the triazolium carboxylate as a white solid (335 mg 84\%). ${ }^{1} \mathrm{H}$ NMR (300 MHz, $\mathrm{CD}_{2} \mathrm{Cl}_{2}$ ): $\delta=7.03$ (s, 2H, $\mathrm{H}_{\mathrm{Mes}}$ ), 4.22 (s, 3H, $\mathrm{NCH}_{3}$ ), 3.30-3.25 (m, $\left.2 \mathrm{H}, \mathrm{C}_{\mathrm{trz}}-\mathrm{CH}_{2}\right), 2.37,1.98\left(2 \times \mathrm{s}, 9 \mathrm{H}, \mathrm{CH}_{3}-\mathrm{C}_{\mathrm{Mes}}\right), 1.79-1.71\left(\mathrm{~m}, 2 \mathrm{H}, \mathrm{C}_{\mathrm{trz}}-\mathrm{CH}_{2}-\mathrm{CH}_{2}\right), 1.54-1.47(\mathrm{~m}, 2 \mathrm{H}$, $\left.\mathrm{CH}_{2}-\mathrm{CH}_{3}\right), 1.45\left(\mathrm{t}, J=7.4 \mathrm{~Hz}, 3 \mathrm{H}, \mathrm{CH}_{2}-\mathrm{CH}_{3}\right) .{ }^{13} \mathrm{C}\left\{{ }^{1} \mathrm{H}\right\} \mathrm{NMR}\left(75 \mathrm{MHz}, \mathrm{CD}_{2} \mathrm{Cl}_{2}\right): \delta=160.56\left(\mathrm{CO}_{2}\right)$, $155.83\left(C-\mathrm{CO}_{2}\right), 143.83\left(\mathrm{C}_{\text {trz }}-\mathrm{C}_{\text {Mes }}\right), 141.60\left(1 \times \mathrm{C}_{\text {Mes }}\right), 134.79\left(2 \times \mathrm{C}_{\text {Mes }}\right), 130.27\left(\mathrm{C}_{\text {Mes }}-\mathrm{N}\right), 129.56$ $\left(\mathrm{C}_{\text {Mes }}-\mathrm{H}\right), 37.99\left(\mathrm{NCH}_{3}\right), 30.71\left(\mathrm{CH}_{2}-\mathrm{CH}_{2}-\mathrm{C}_{\text {trz }}\right), 23.96\left(\mathrm{CH}_{2}-\mathrm{C}_{\text {trz }}\right), 22.97\left(\mathrm{CH}_{2}-\mathrm{CH}_{3}\right), 21.52\left(\mathrm{CH}_{3}-\right.$ $\left.\mathrm{C}_{\text {Mes }}\right), 17.60\left(2 \times \mathrm{CH}_{3}-\mathrm{C}_{\mathrm{Mes}}\right), 14.06\left(\mathrm{CH}_{3}-\mathrm{CH}_{2}\right)$. IR $\left(\mathrm{CH}_{2} \mathrm{Cl}_{2}, \mathrm{~cm}^{-1}\right): 1658 \mathrm{~cm}^{-1} v\left(\mathrm{CO}_{2}\right)$. HRMS: m/z: calcd. for $\mathrm{C}_{17} \mathrm{H}_{23} \mathrm{~N}_{3} \mathrm{O}_{2}[\mathrm{M}+\mathrm{H}]^{+}: 302.1863$; found: 302.1850 . 


\section{NMR spectra of all new compounds}

$1 e^{1} H_{N M R}$
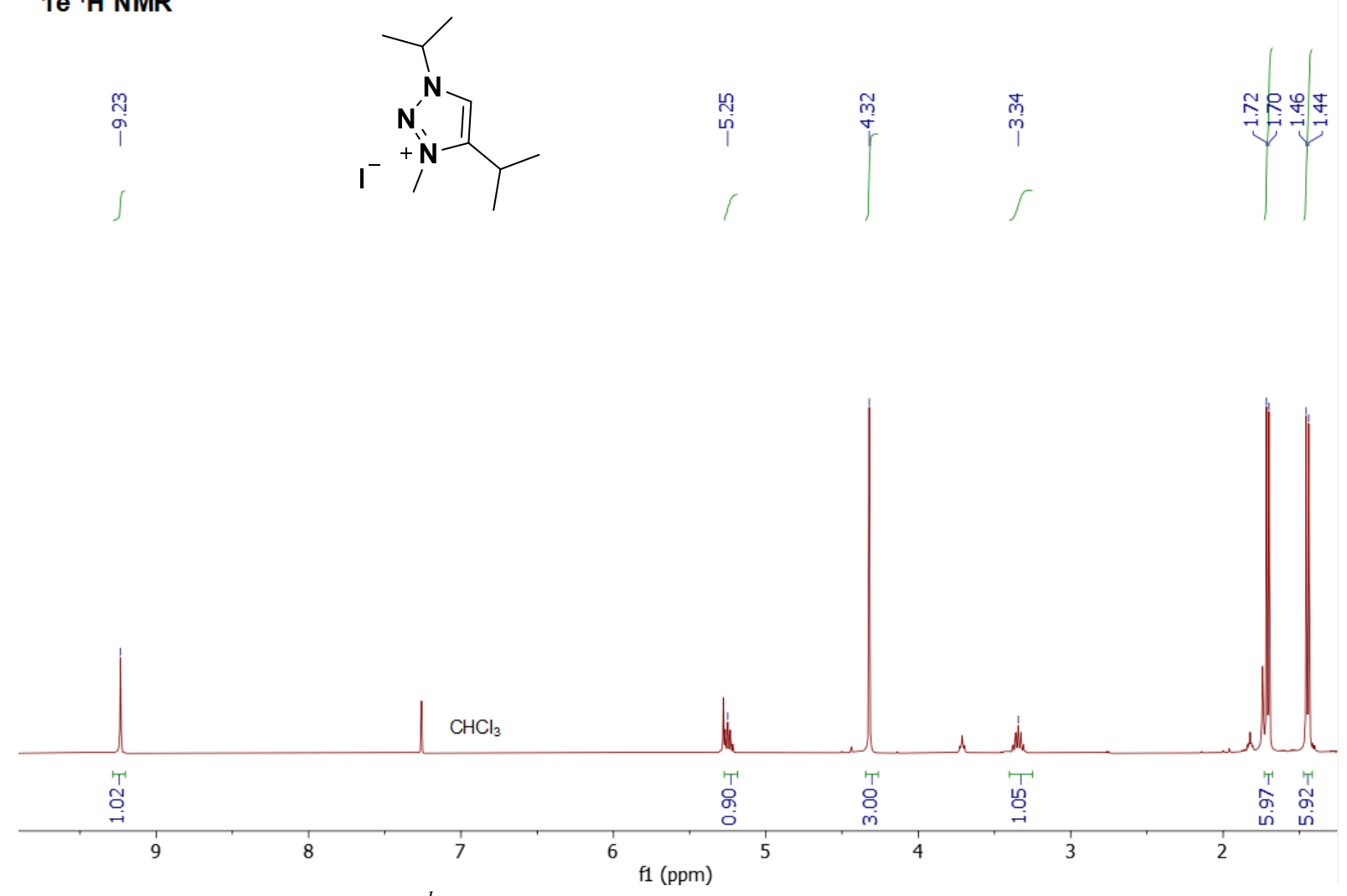

Figure S1. ${ }^{1} \mathrm{H} \mathrm{NMR}$ spectrum (400 $\left.\mathrm{MHz}, \mathrm{CDCl}_{3}\right)$ for ligand 1e

$1 \mathrm{e}^{13} \mathrm{C}\left\{{ }^{1} \mathrm{H}\right\}$ NMR

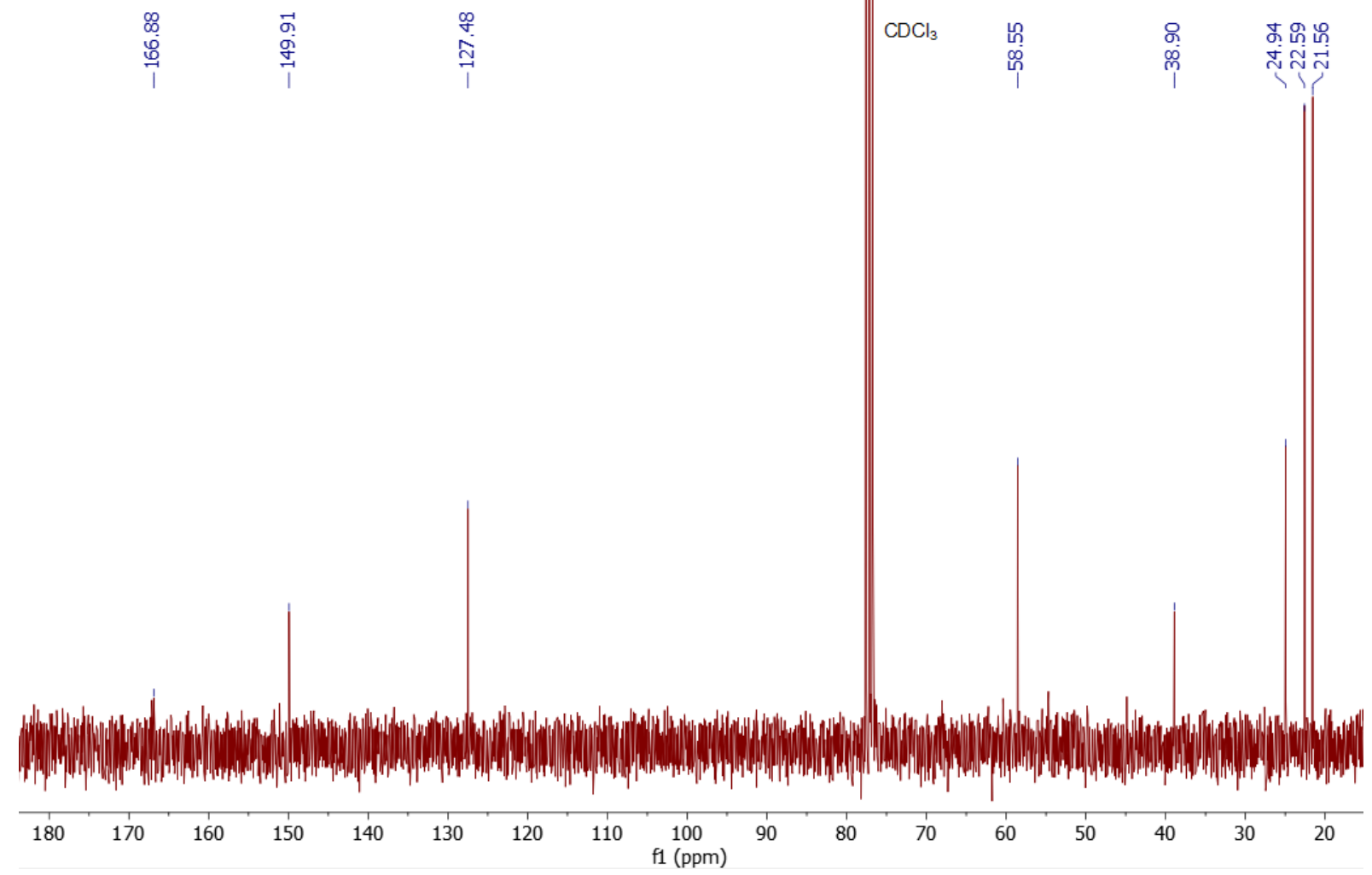

Figure S2. $\left.{ }^{13} C_{\{}{ }^{1} H\right\} N M R$ spectrum $\left(101 \mathrm{MHz}, \mathrm{CDCl}_{3}\right)$ for $1 \boldsymbol{e}$ 


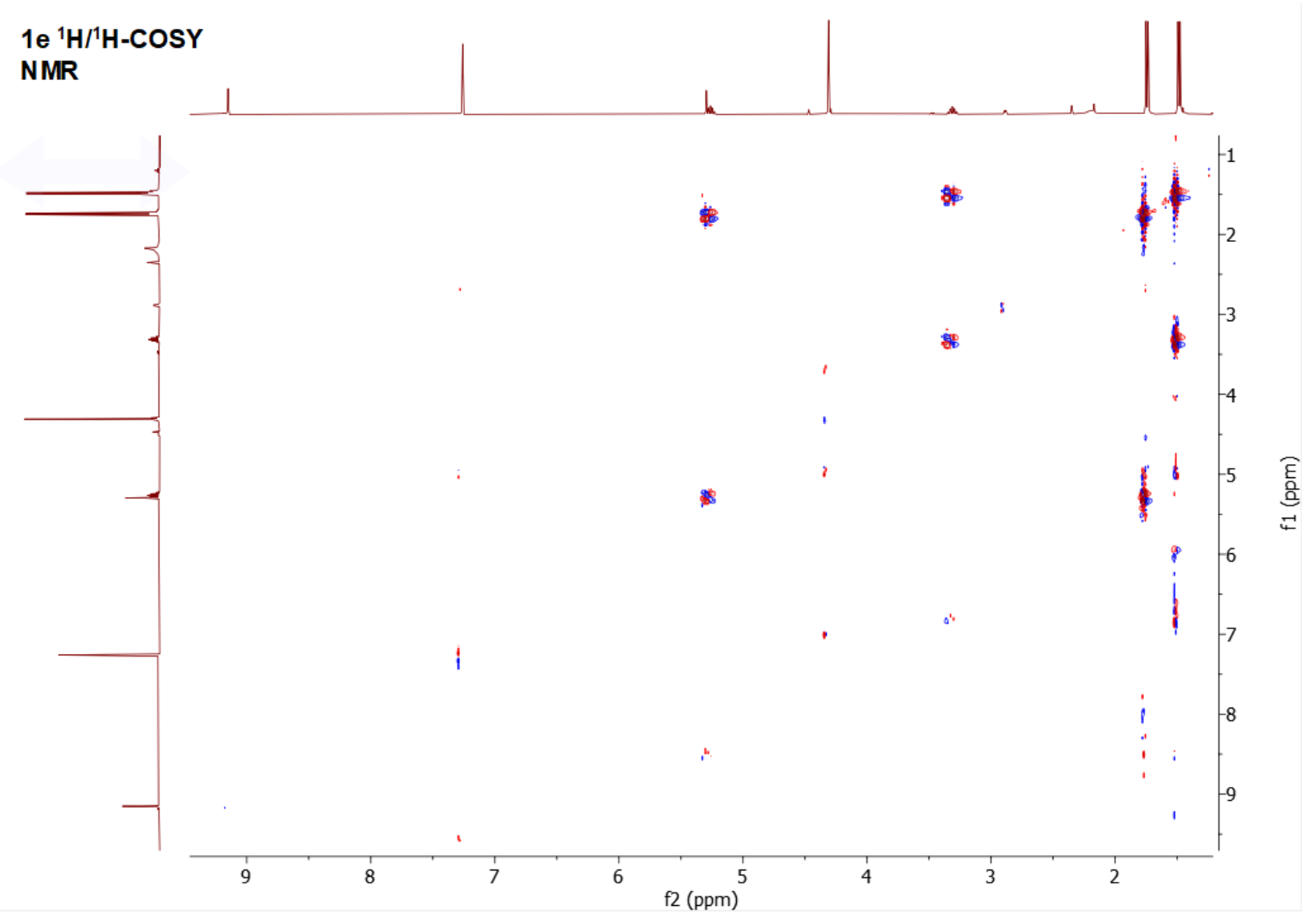

Figure S3. ${ }^{l} \mathrm{H} /{ }^{l} \mathrm{H}$-COSY NMR spectrum $\left(300 \mathrm{MHz}, \mathrm{CDCl}_{3}\right)$ for $\mathbf{1 e}$.

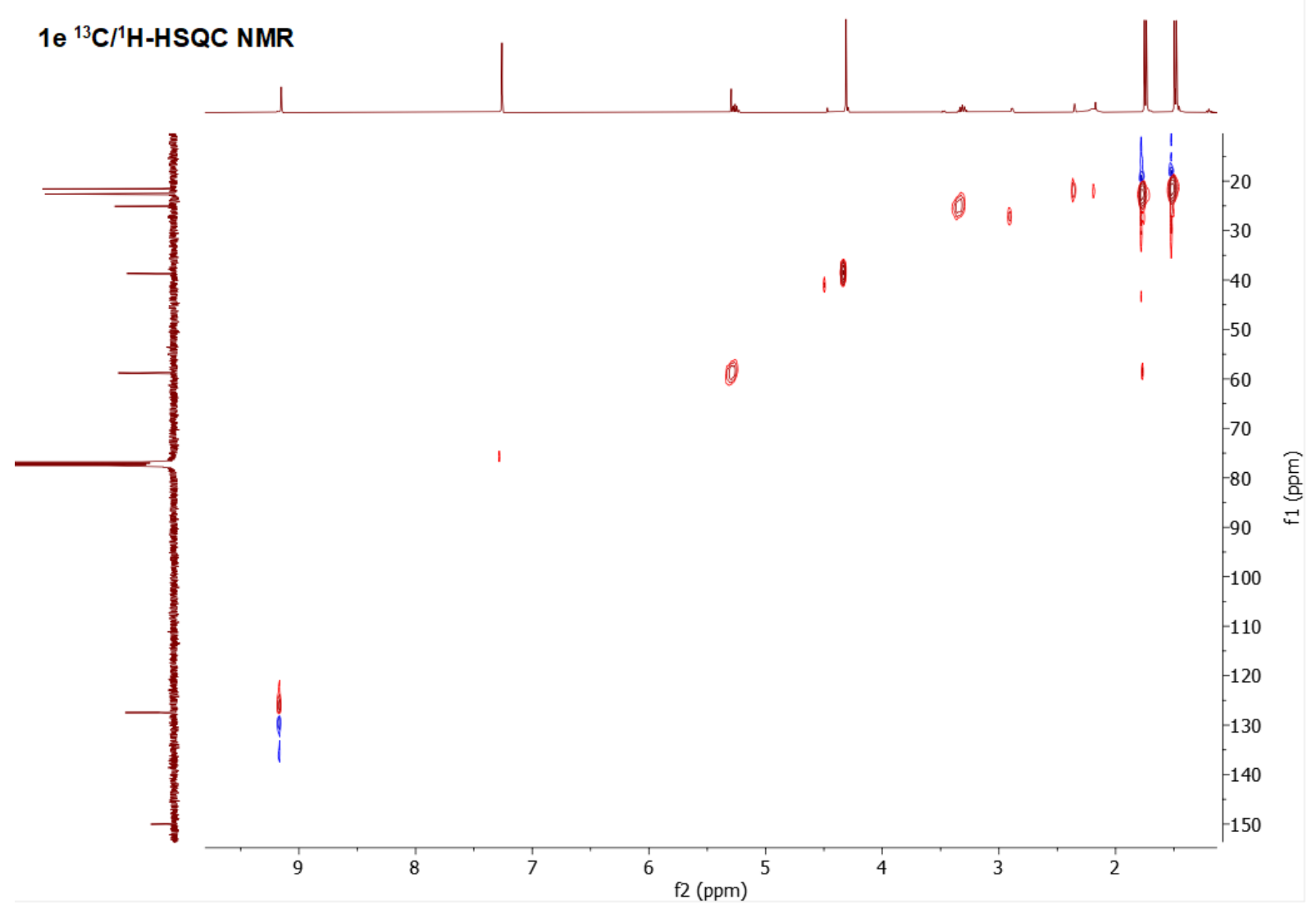

Figure S4. ${ }^{13} \mathrm{C} / \mathrm{H}$-HSQC NMR spectrum $\left(\mathrm{CDCl}_{3}\right)$ for $\mathbf{l e}$ 


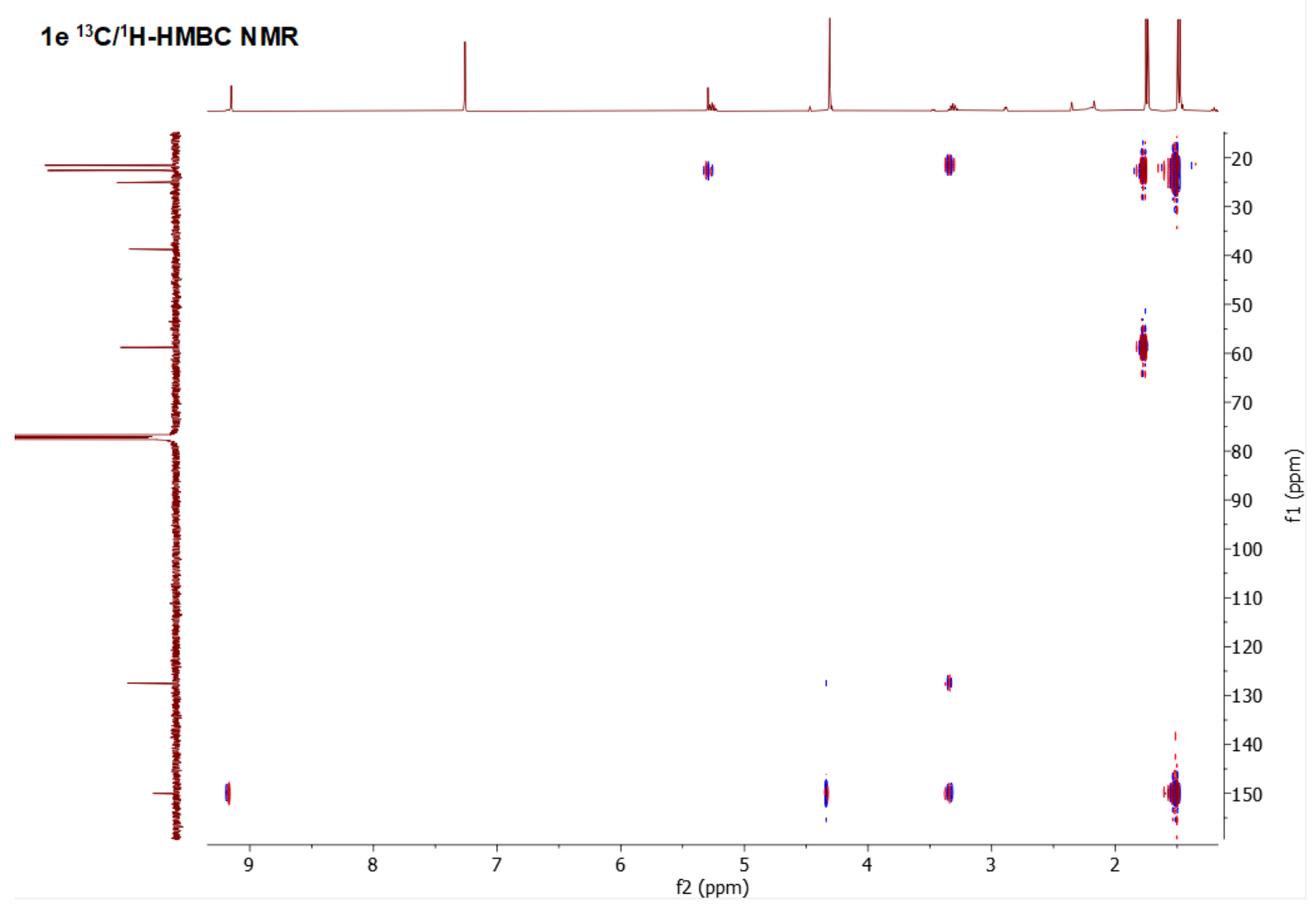

Figure S5. ${ }^{13} \mathrm{C} /{ }^{1} \mathrm{H}-\mathrm{HMBC} \mathrm{NMR}$ spectrum $\left(\mathrm{CDCl}_{3}\right)$ for $\mathbf{1 e}$

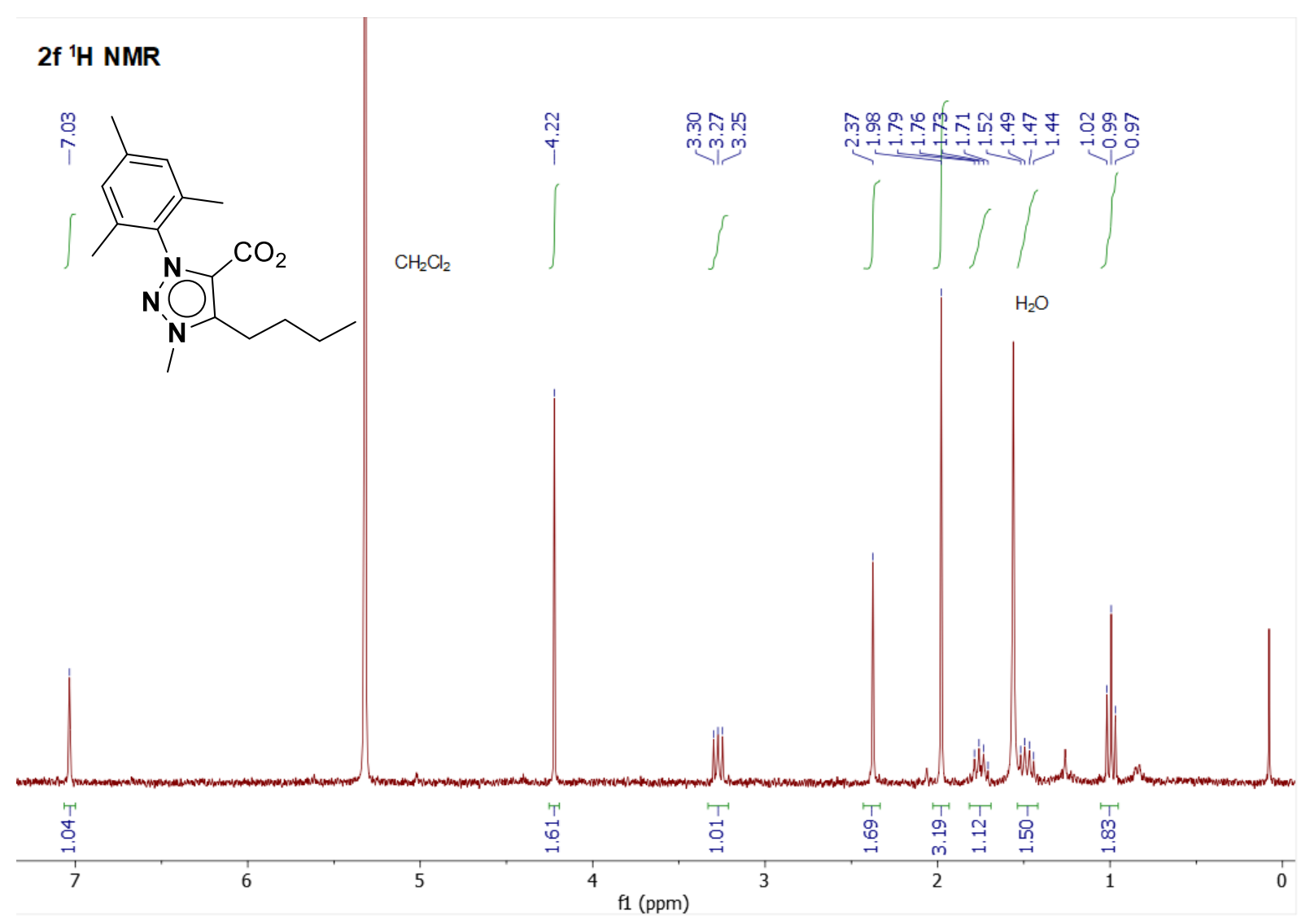

Figure S6. ${ }^{1} \mathrm{H} \mathrm{NMR}$ spectrum $\left(300 \mathrm{MHz}, \mathrm{CD}_{2} \mathrm{Cl}_{2}\right)$ for $\boldsymbol{2} \boldsymbol{f}$ 


\section{$2 f{ }^{13} \mathrm{C}\left\{{ }^{1} \mathrm{H}\right\}$ NMR}

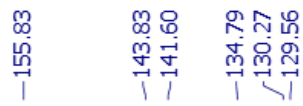

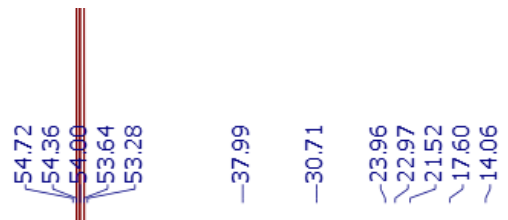

$\mathrm{CDCl}_{3}$

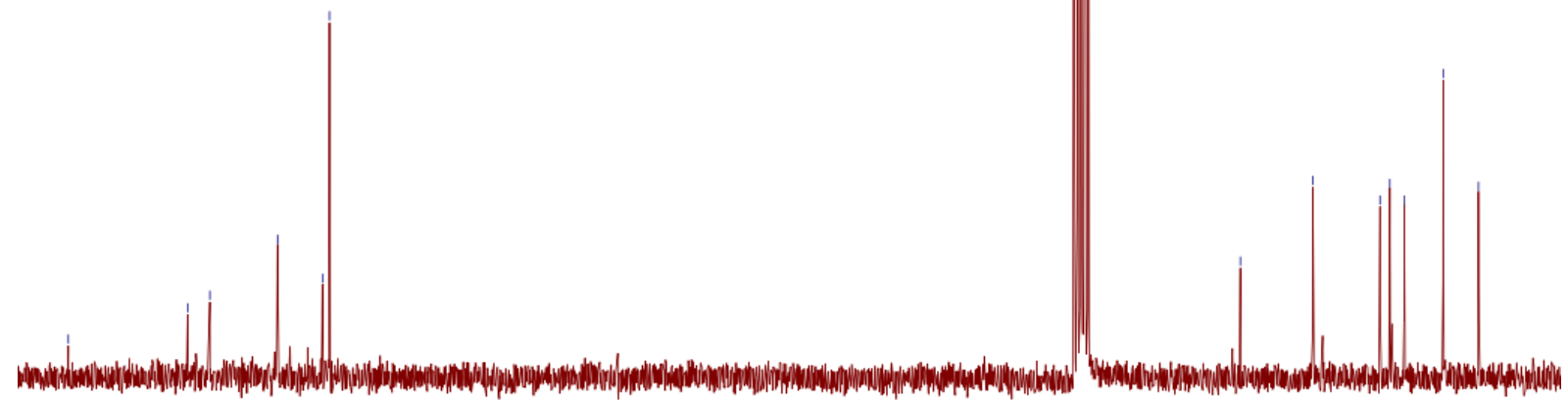

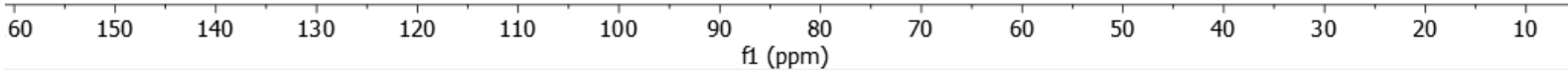

Figure S7. ${ }^{13} \mathrm{C}_{\left\{{ }^{1} \mathrm{H}\right\}} \mathrm{NMR}$ spectrum $\left(75 \mathrm{MHz}, \mathrm{CD}_{2} \mathrm{Cl}_{2}\right)$ for $2 \boldsymbol{f}$

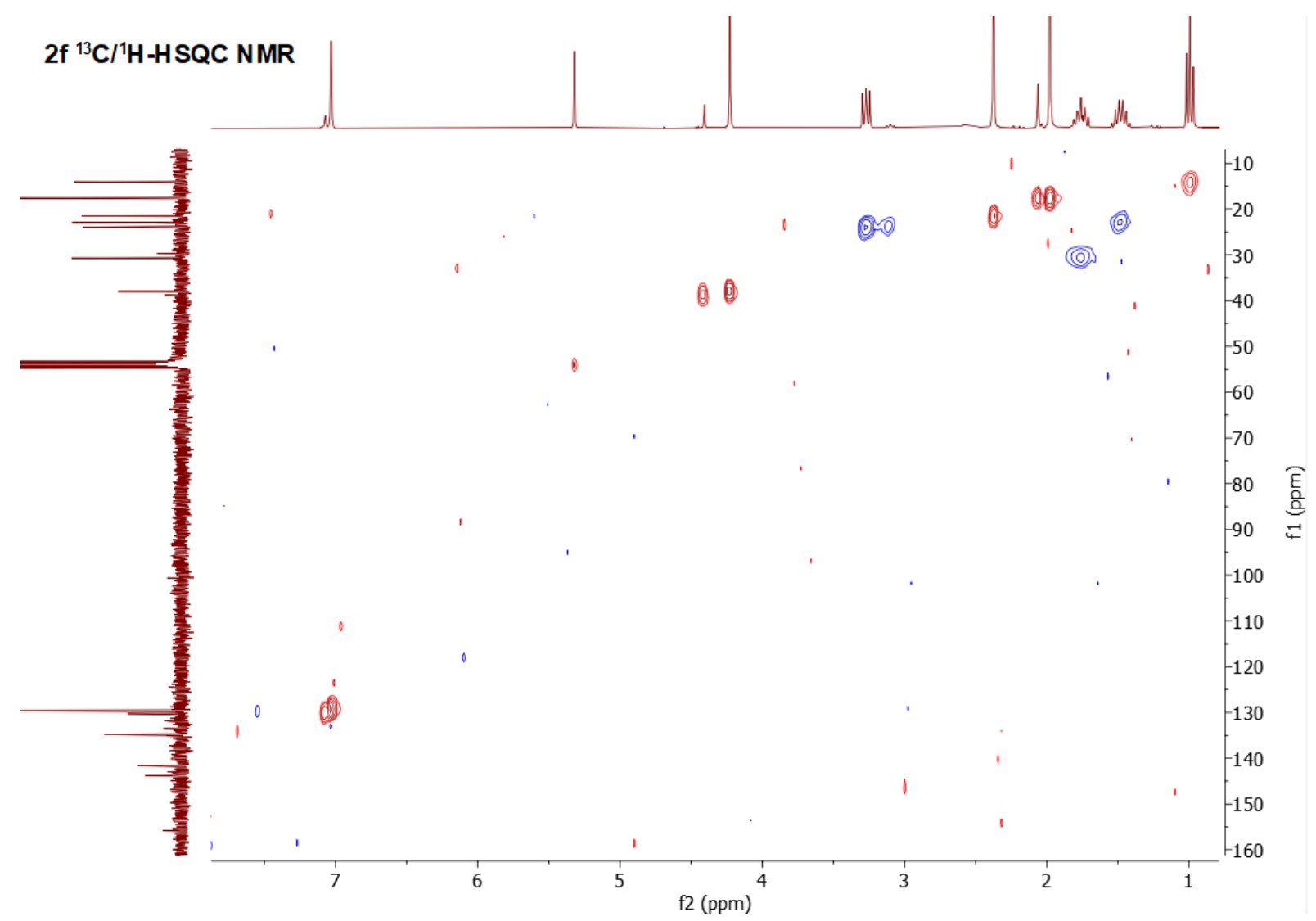

Figure S8. ${ }^{13} \mathrm{C} /{ }^{1} \mathrm{H}$-HSQC NMR spectrum $\left(\mathrm{CD}_{2} \mathrm{Cl}_{2}\right)$ for $2 \boldsymbol{f}$. 


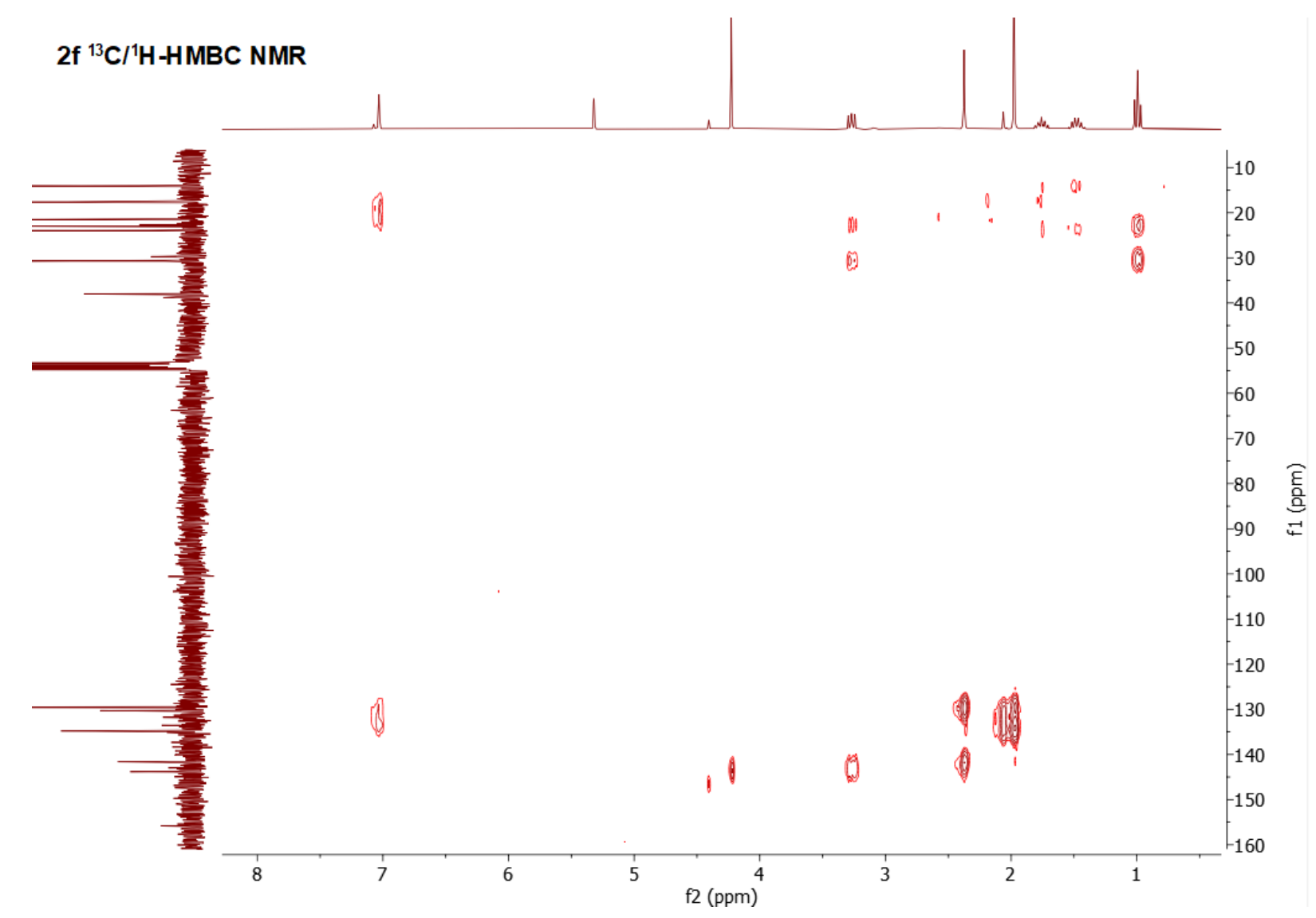

Figure S9. ${ }^{13} \mathrm{C} /{ }^{1} \mathrm{H}-\mathrm{HMBC} \mathrm{NMR}$ spectrum $\left(\mathrm{CD}_{2} \mathrm{Cl}_{2}\right)$ for $2 \mathrm{f}$.

$3 c^{1} \mathrm{H}$ NMR

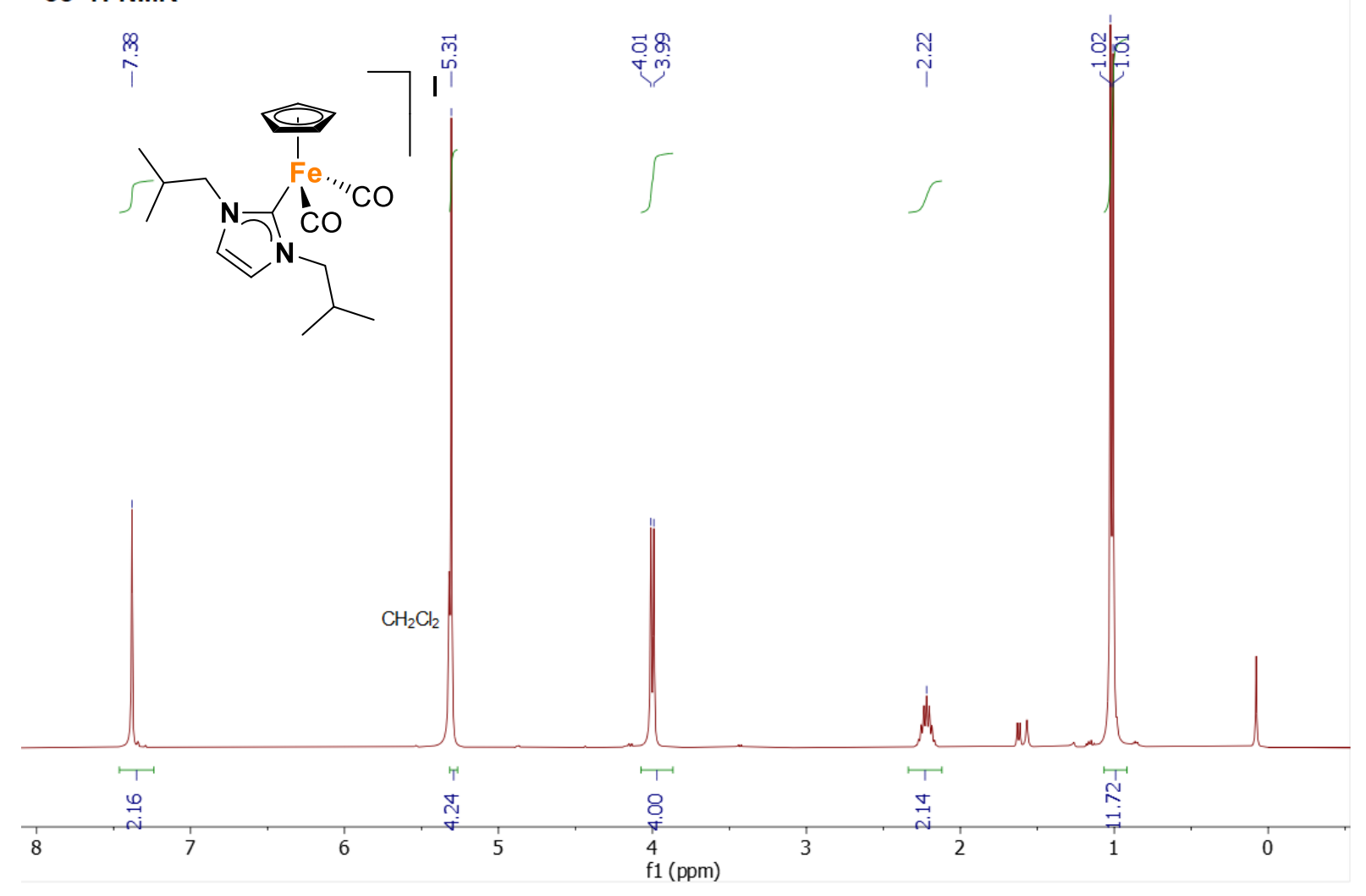

Figure S10. ${ }^{1} \mathrm{H} N M R$ spectrum $\left(400 \mathrm{MHz}, \mathrm{CD}_{2} \mathrm{Cl}_{2}\right)$ for complex $3 \mathrm{c}$. 
$3 \mathrm{c}{ }^{13} \mathrm{C}\left\{{ }^{1} \mathrm{H}\right\}$ NMR

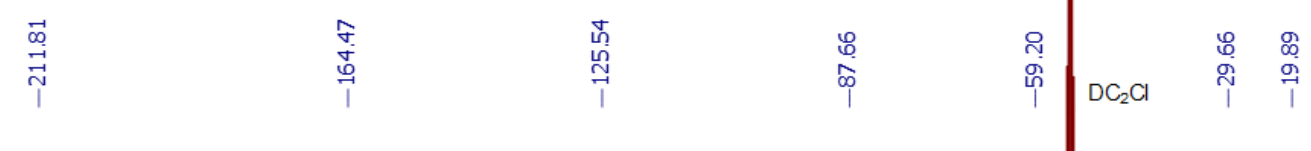

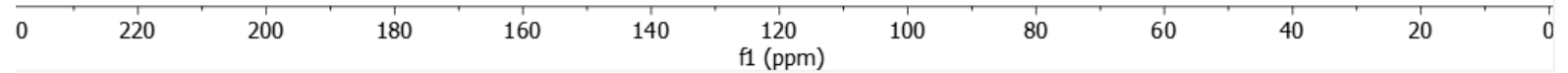

Figure S11. $\left.{ }^{13} \mathrm{C}_{\{}{ }^{1} \mathrm{H}\right\}$ NMR spectrum $\left(101 \mathrm{MHz}, \mathrm{CD}_{2} \mathrm{Cl}_{2}\right)$ for complex $3 \boldsymbol{c}$.

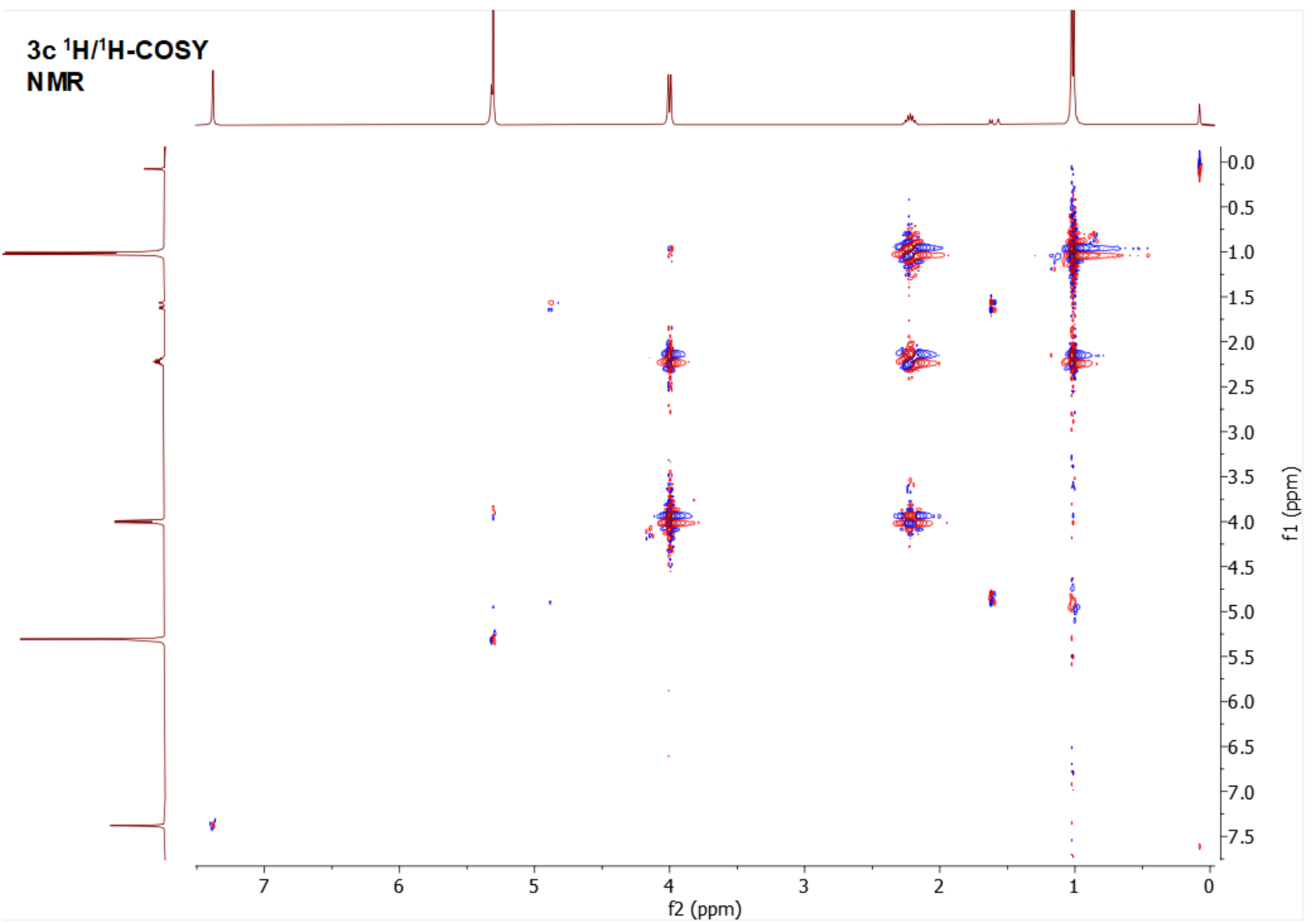

Figure S12. ${ }^{l} \mathrm{H} /{ }^{l} \mathrm{H}$-COSY NMR spectrum $\left(400 \mathrm{MHz}, \mathrm{CD}_{2} \mathrm{Cl}_{2}\right.$ ) for complex $3 \mathrm{c}$ 


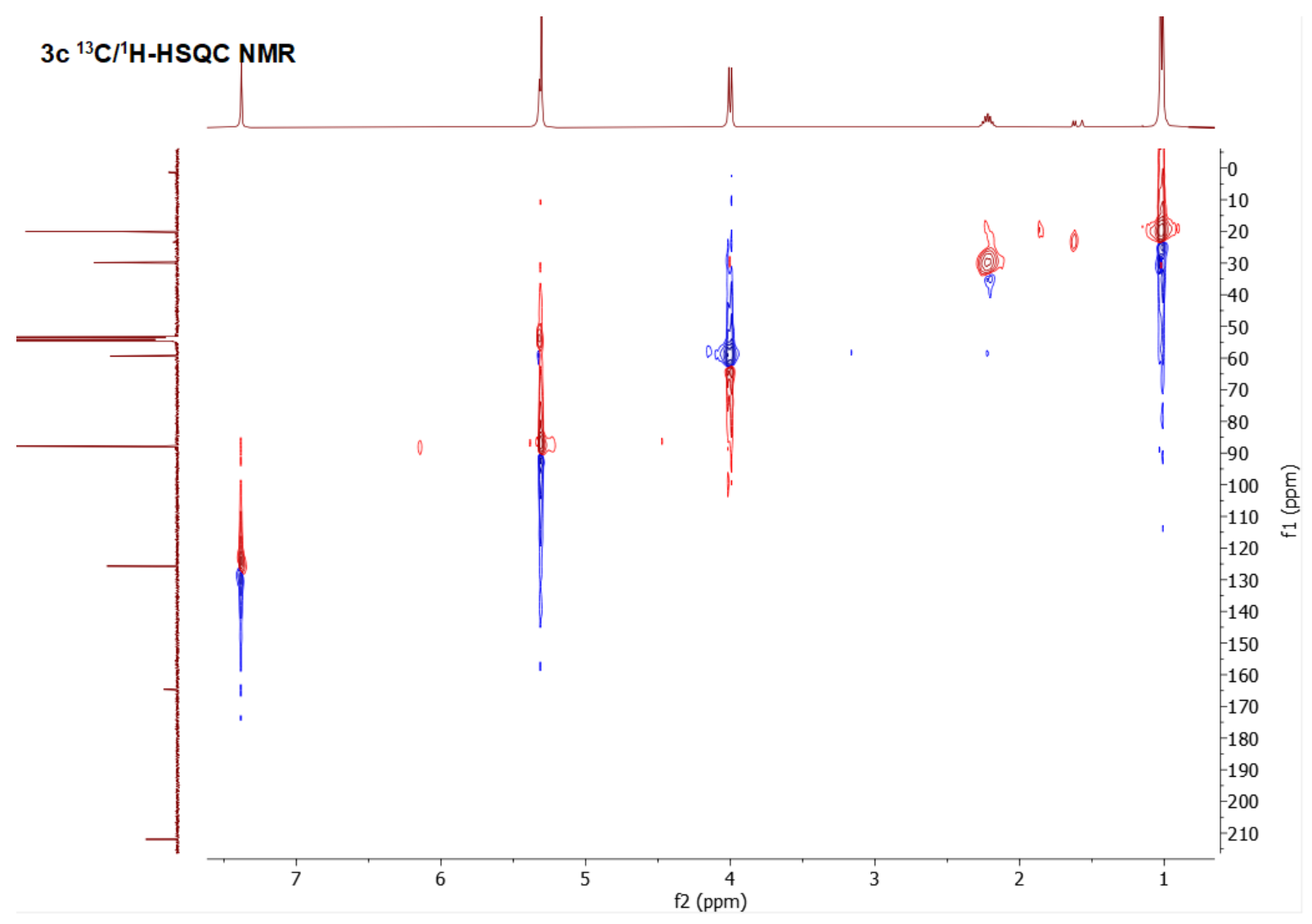

Figure S13. ${ }^{13} \mathrm{C} /{ }^{1} \mathrm{H}-\mathrm{HSQC}$ NMR spectrum $\left(\mathrm{CD}_{2} \mathrm{Cl}_{2}\right)$ for complex $3 \mathrm{c}$.

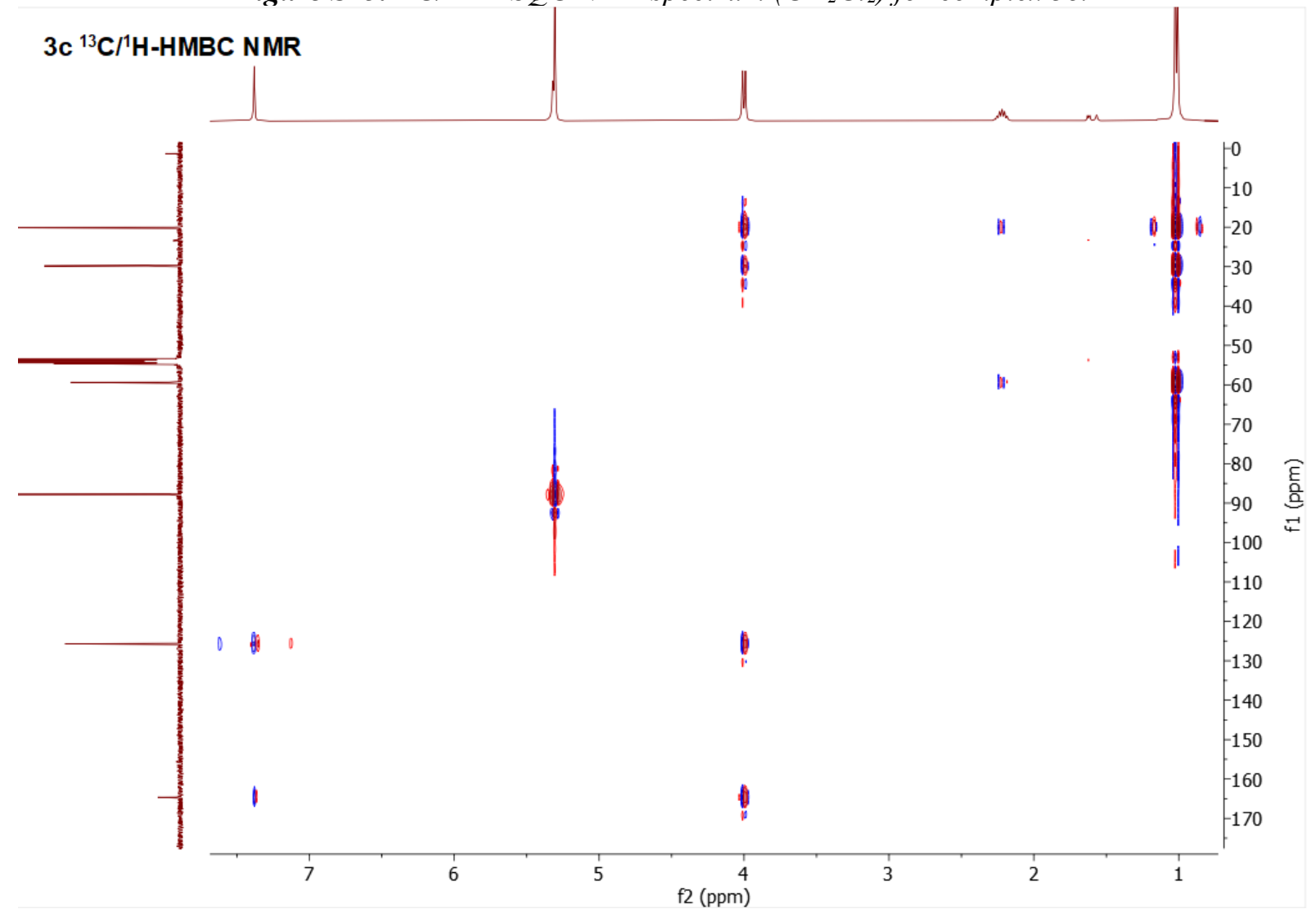

Figure S14. ${ }^{13} \mathrm{C} /{ }^{1} \mathrm{H}-\mathrm{HMBC}$ NMR spectrum $\left(\mathrm{CD}_{2} \mathrm{Cl}_{2}\right)$ for complex $3 \boldsymbol{c}$. 


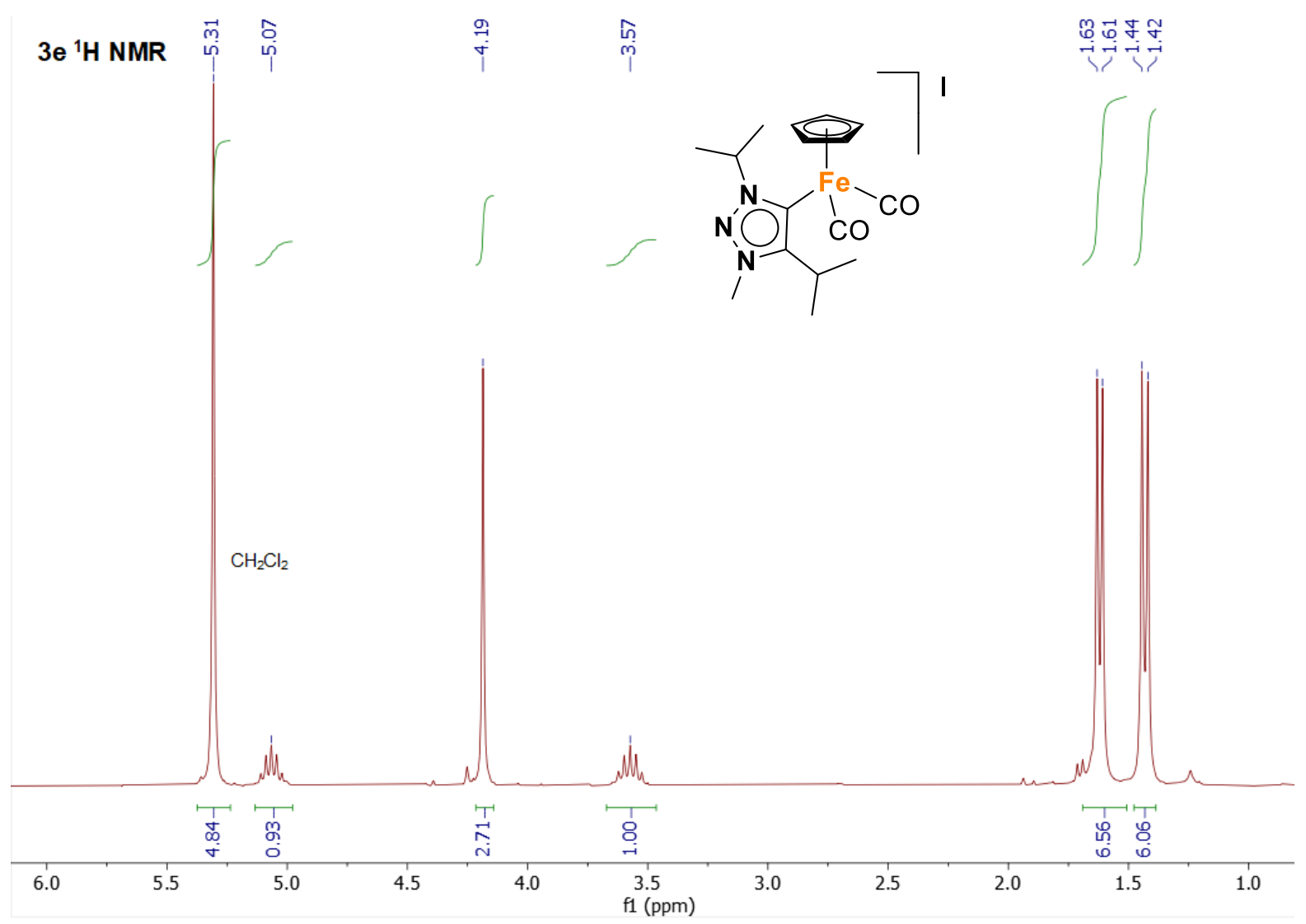

Figure S15. ${ }^{1} \mathrm{H}$ NMR spectrum $\left(300 \mathrm{MHz}, \mathrm{CD}_{2} \mathrm{Cl}_{2}\right)$ for complex $3 \boldsymbol{e}$.

\section{$3 e^{13} \mathrm{C}\left\{{ }^{1} \mathrm{H}\right\}$ NMR}

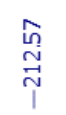
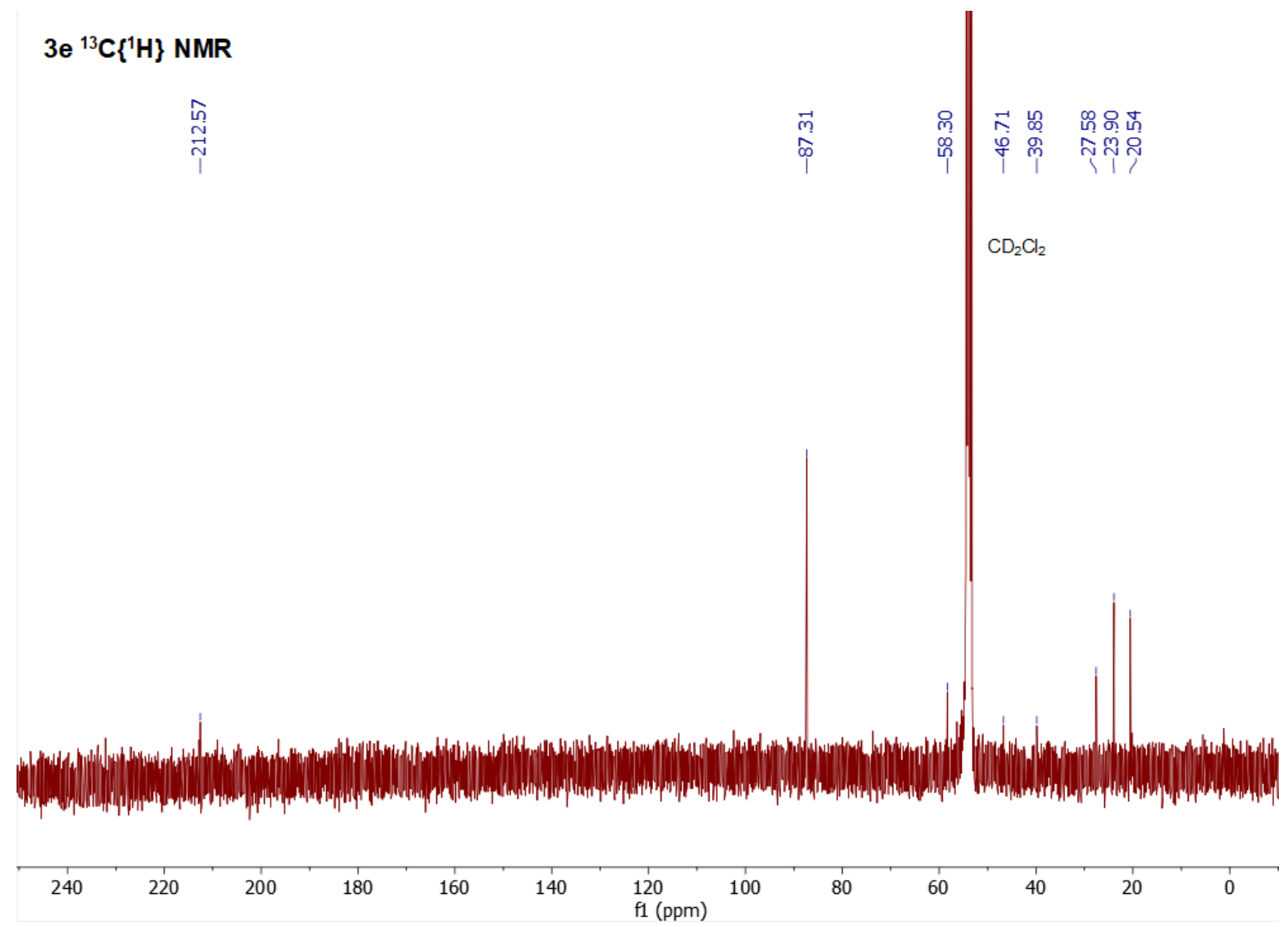

Figure S16. $\left.{ }^{13} \mathrm{C}_{\{}{ }^{1} \mathrm{H}\right\}$ NMR spectrum $\left(101 \mathrm{MHz}, \mathrm{CD}_{2} \mathrm{Cl}_{2}\right)$ for complex $3 \boldsymbol{e}$. 


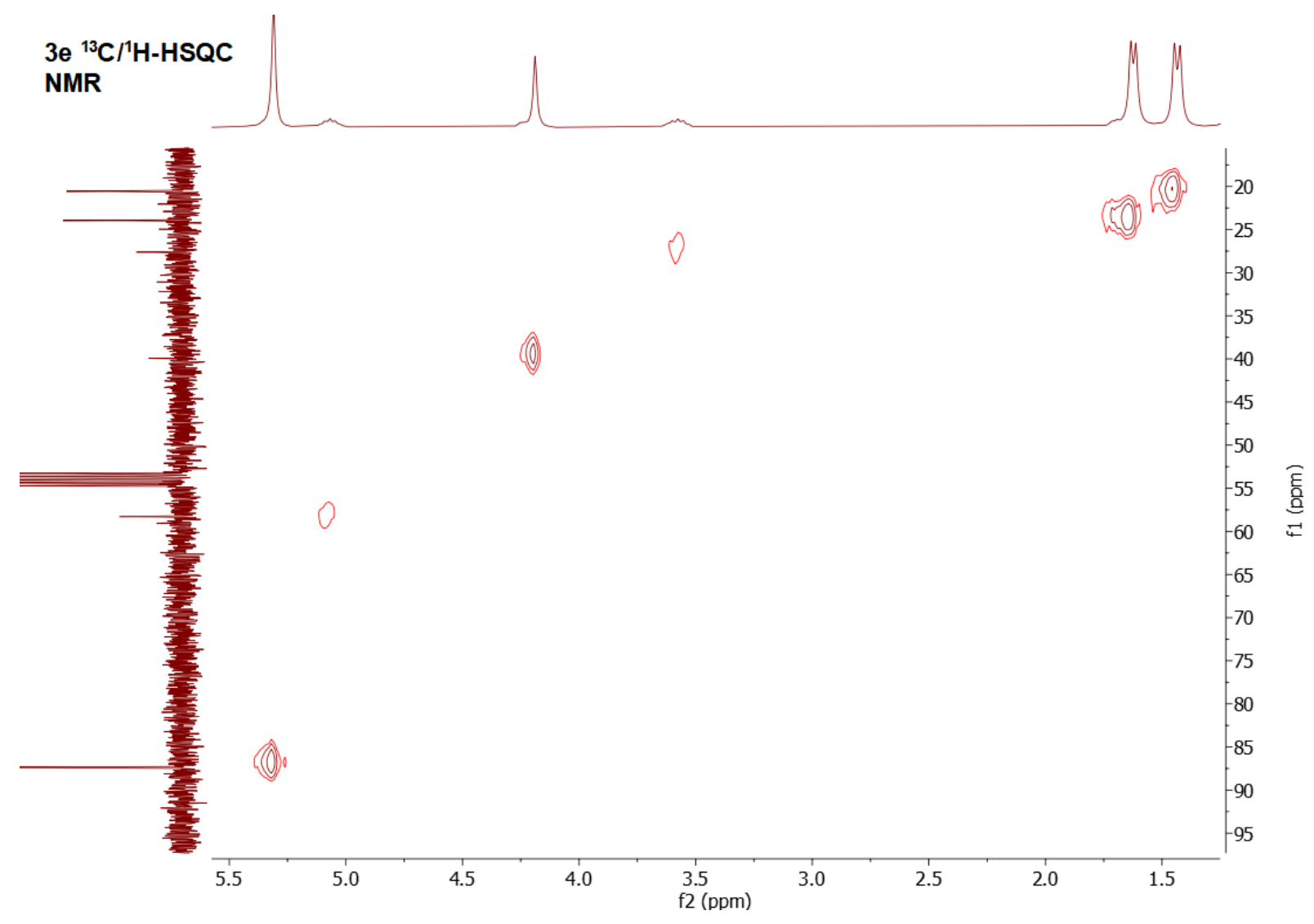

Figure S17. ${ }^{13} \mathrm{C} /{ }^{1} \mathrm{H}-\mathrm{HSQC}$ NMR spectrum $\left(\mathrm{CD}_{2} \mathrm{Cl}_{2}\right)$ for complex $3 \boldsymbol{e}$.
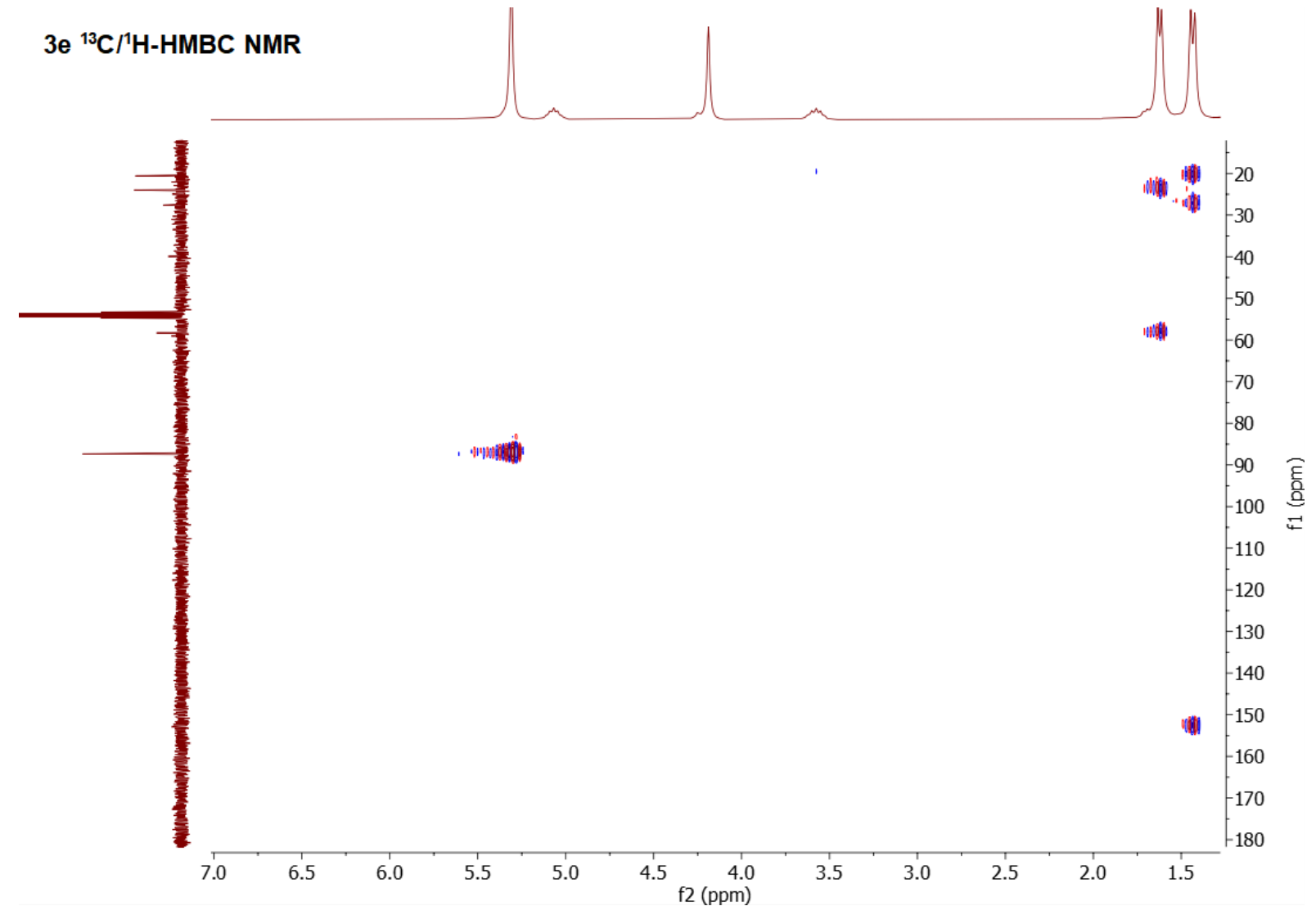

Figure S18. ${ }^{13} \mathrm{C} /{ }^{1} \mathrm{H}-\mathrm{HMBC} N \mathrm{NMR}$ spectrum $\left(\mathrm{CD}_{2} \mathrm{Cl}_{2}\right)$ for complex $3 \boldsymbol{e}$. 
4a ${ }^{1}$ H NMR
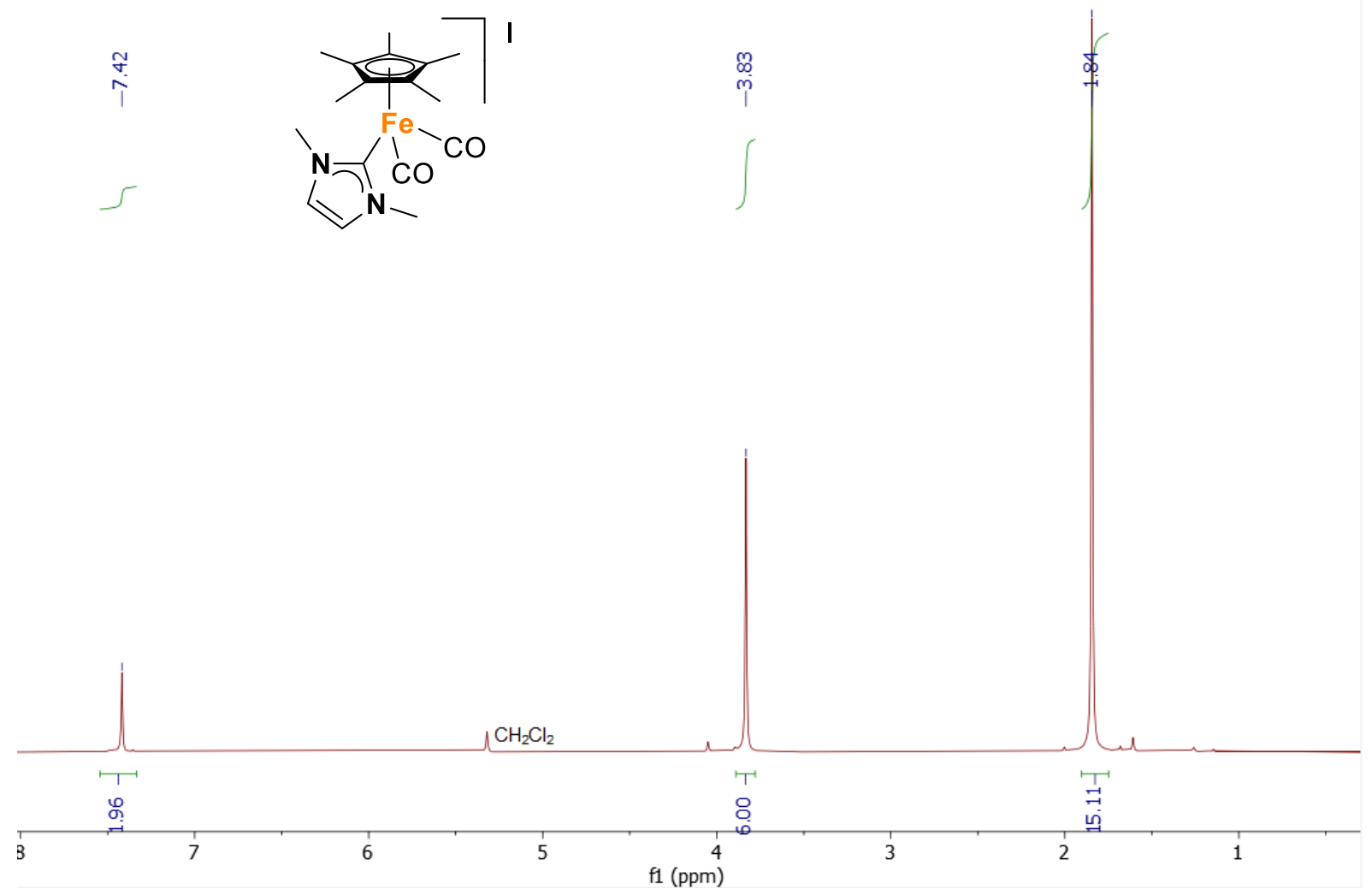

Figure S19. ${ }^{1} \mathrm{H} N \mathrm{NR}$ spectrum $\left(400 \mathrm{MHz}, \mathrm{CD}_{2} \mathrm{Cl}_{2}\right)$ for complex $4 \boldsymbol{a}$

\section{4a ${ }^{13} \mathrm{C}\left\{{ }^{1} \mathrm{H}\right\}$ NMR}

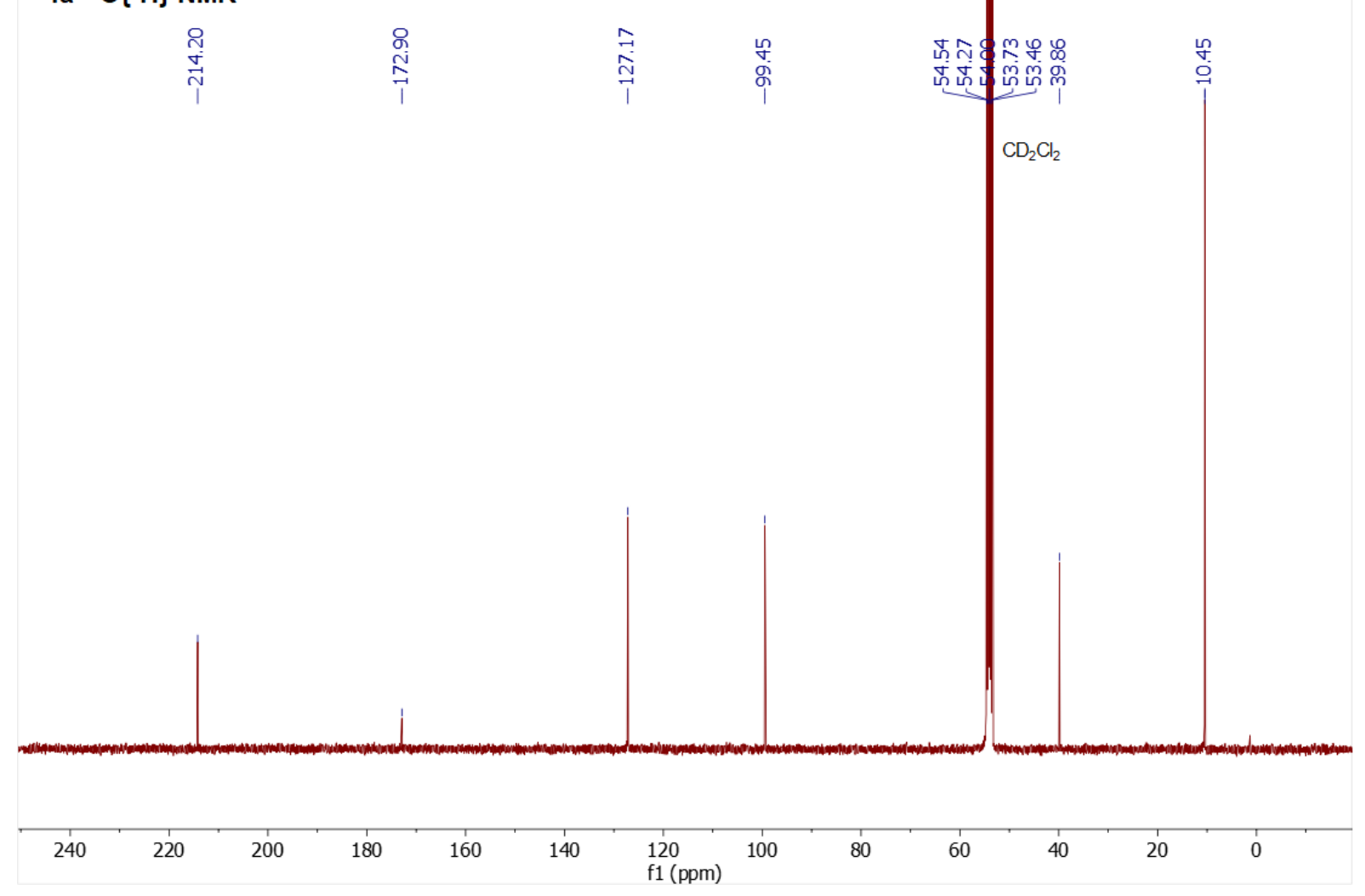

Figure S20. ${ }^{13} C_{\{}^{\{l} \mathrm{H}_{\}} \mathrm{NMR}$ spectrum $\left(101 \mathrm{MHz}, \mathrm{CD}_{2} \mathrm{Cl}_{2}\right)$ for complex $4 \boldsymbol{a}$ 
$4 a^{13} \mathrm{C} /{ }^{1} \mathrm{H}-\mathrm{HMBC}$ NMR

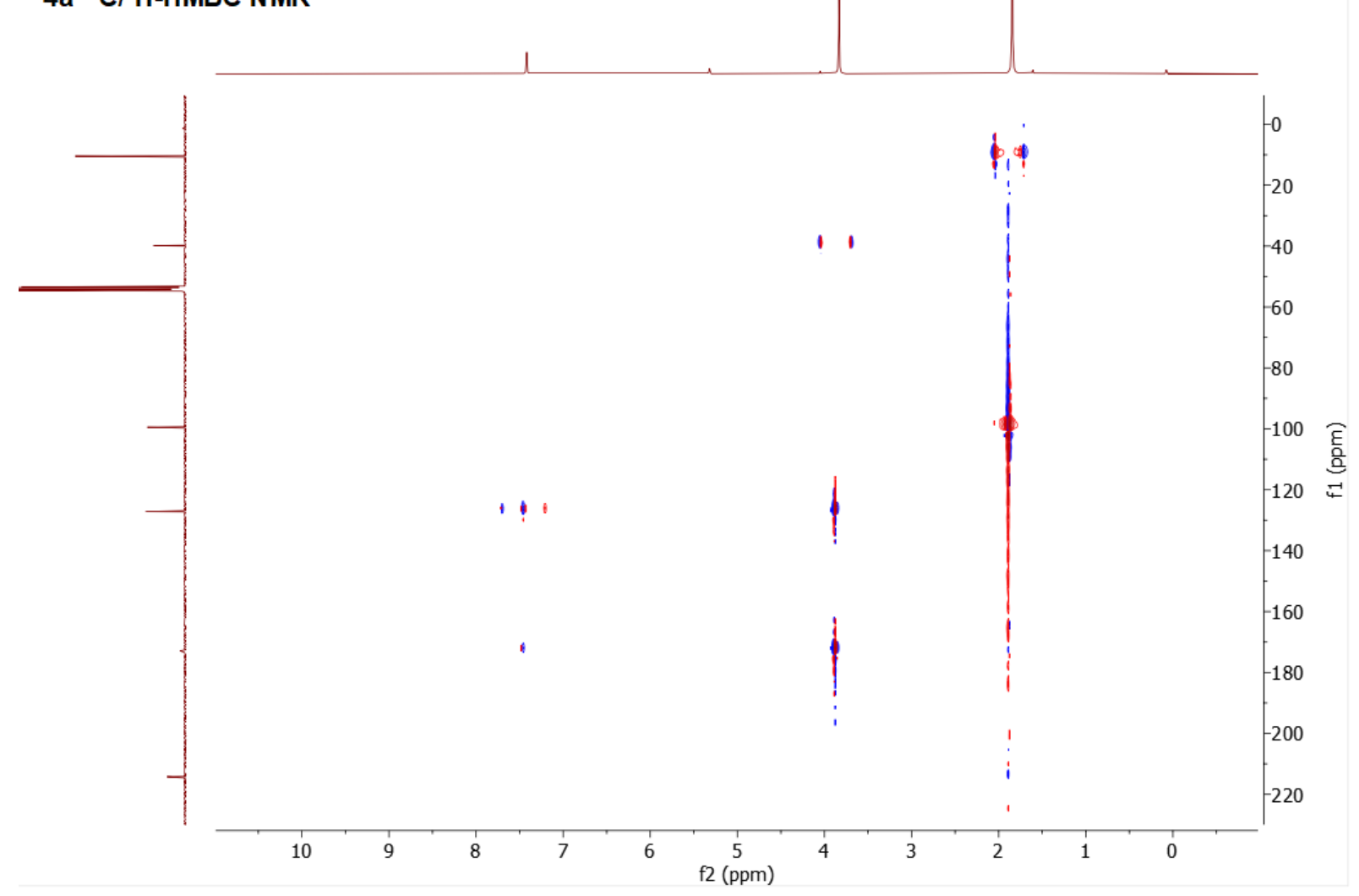

Figure S21. ${ }^{13} \mathrm{C} /{ }^{1} \mathrm{H}-\mathrm{HMBC} \mathrm{NMR}$ spectrum $\left(\mathrm{CD}_{2} \mathrm{Cl}_{2}\right)$ for complex $\mathbf{4 a}$

\section{4b ${ }^{1} \mathrm{H}$ NMR}

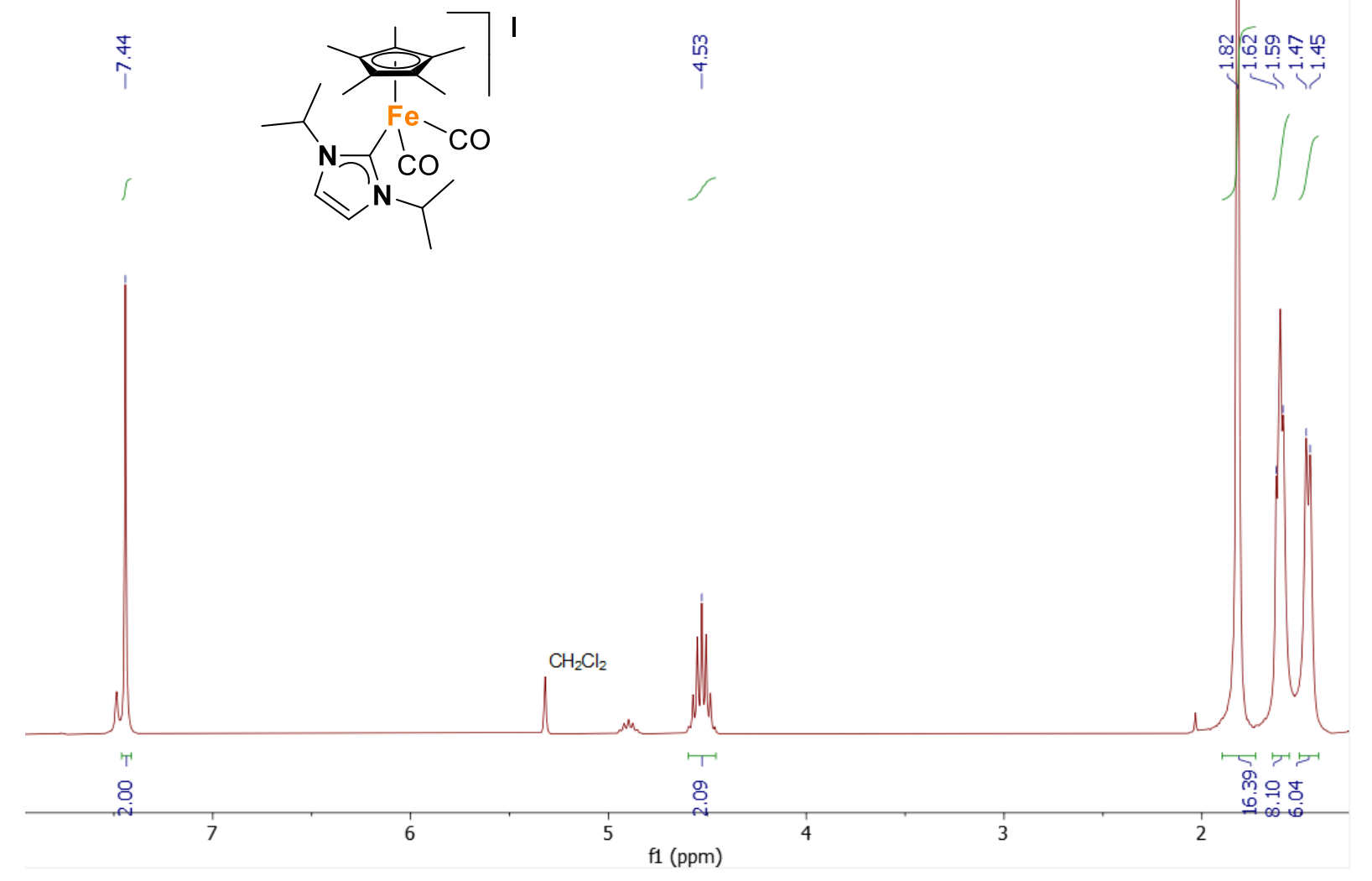

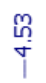

Figure S22. ${ }^{1} \mathrm{H} N \mathrm{NR}$ spectrum $\left(300 \mathrm{MHz}, \mathrm{CD}_{2} \mathrm{Cl}_{2}\right)$ for complex $\mathbf{4 b}$. 
$4 b{ }^{13} \mathrm{C}\left\{{ }^{1} \mathrm{H}\right\}$ NMR

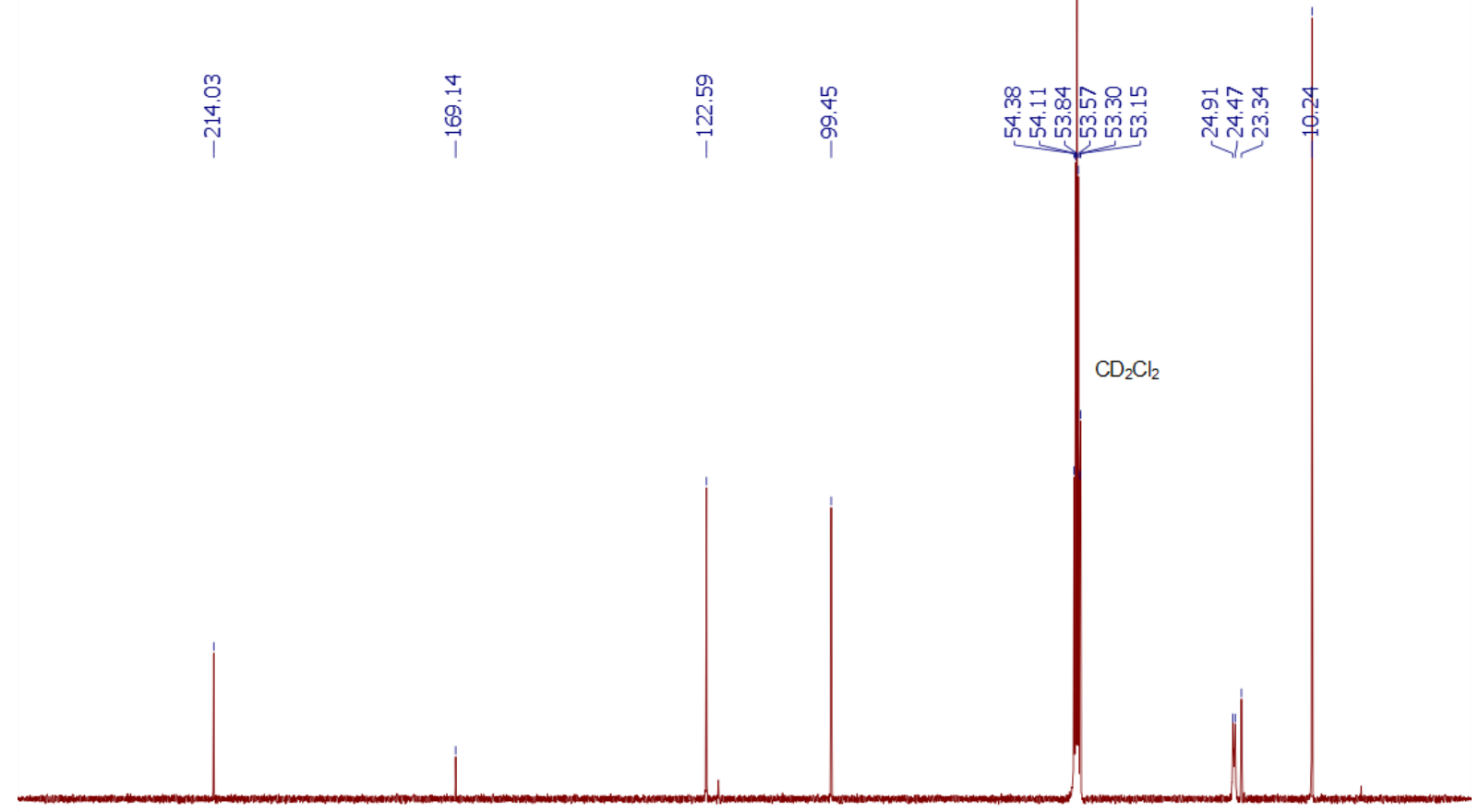

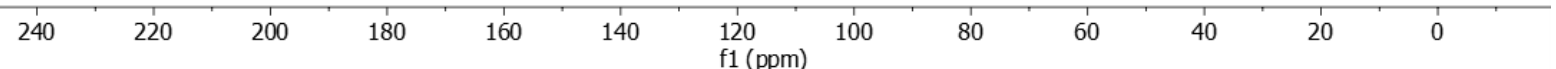

Figure S23. $\left.{ }^{13} \mathrm{C}_{\{}{ }^{1} \mathrm{H}\right\} \mathrm{NMR}$ spectrum $\left(101 \mathrm{MHz}, \mathrm{CD}_{2} \mathrm{Cl}_{2}\right)$ for complex $\mathbf{4 b}$.

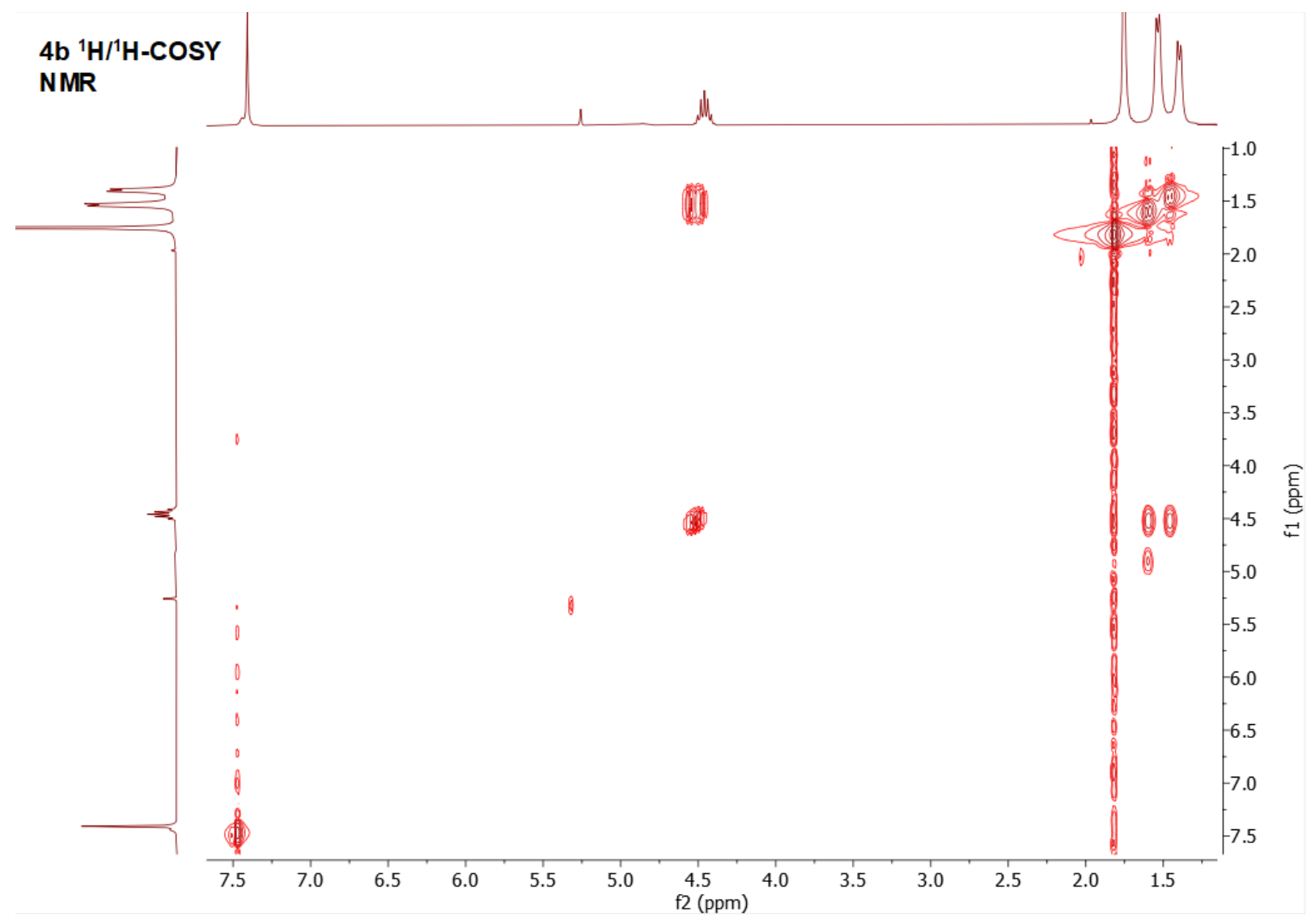

Figure S24. ${ }^{1} \mathrm{H} /{ }^{l} \mathrm{H}$-COSY NMR spectrum $\left(400 \mathrm{MHz}, \mathrm{CD}_{2} \mathrm{Cl}_{2}\right)$ for complex $4 \boldsymbol{b}$. 
$4 b{ }^{13} \mathrm{C} /{ }^{1} \mathrm{H}-\mathrm{HSQC}$ NMR

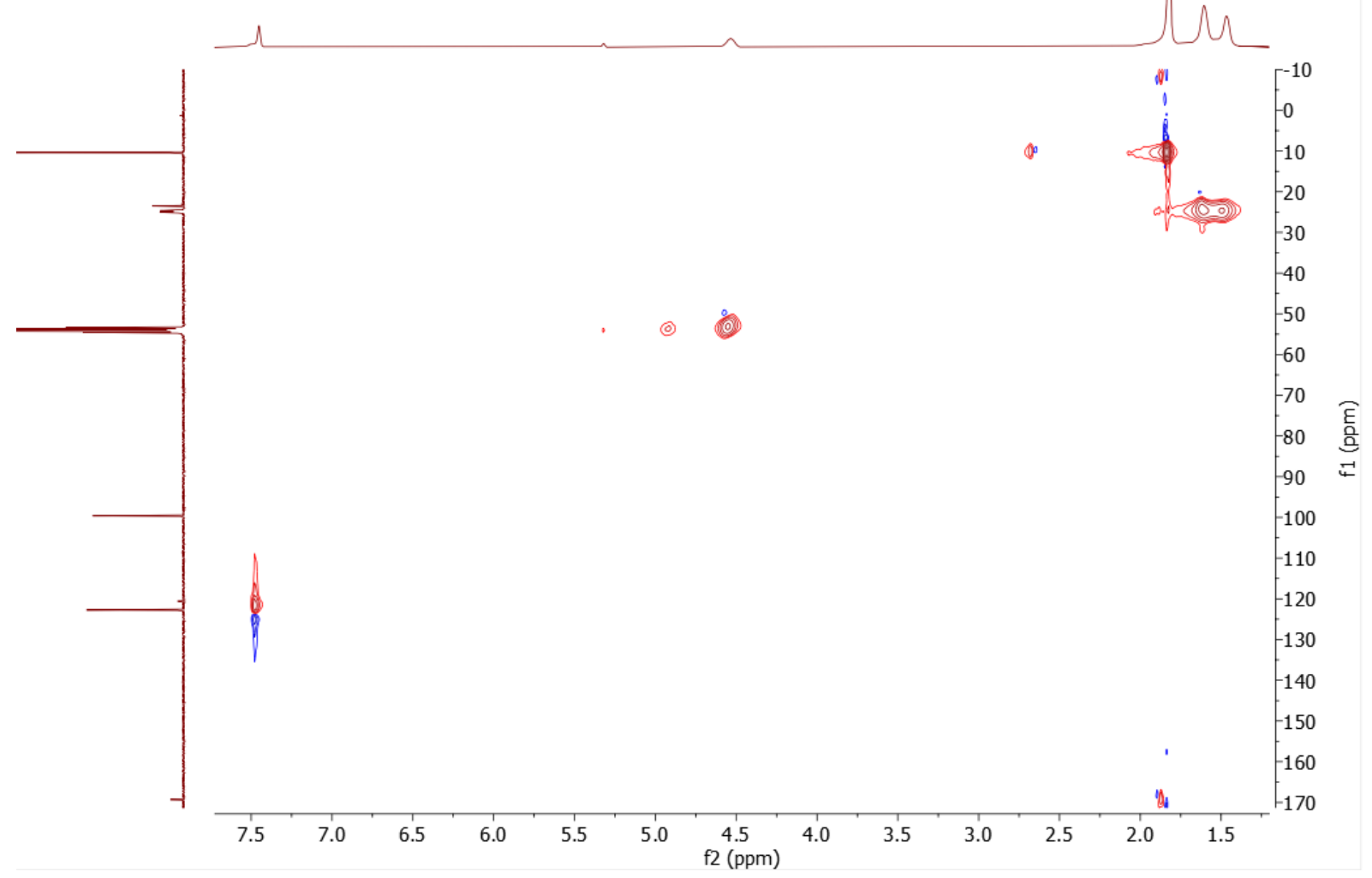

Figure S25. ${ }^{13} \mathrm{C} /{ }^{1} \mathrm{H}$-HSQC NMR spectrum $\left(\mathrm{CD}_{2} \mathrm{Cl}_{2}\right)$ for complex $\mathbf{4 b}$.

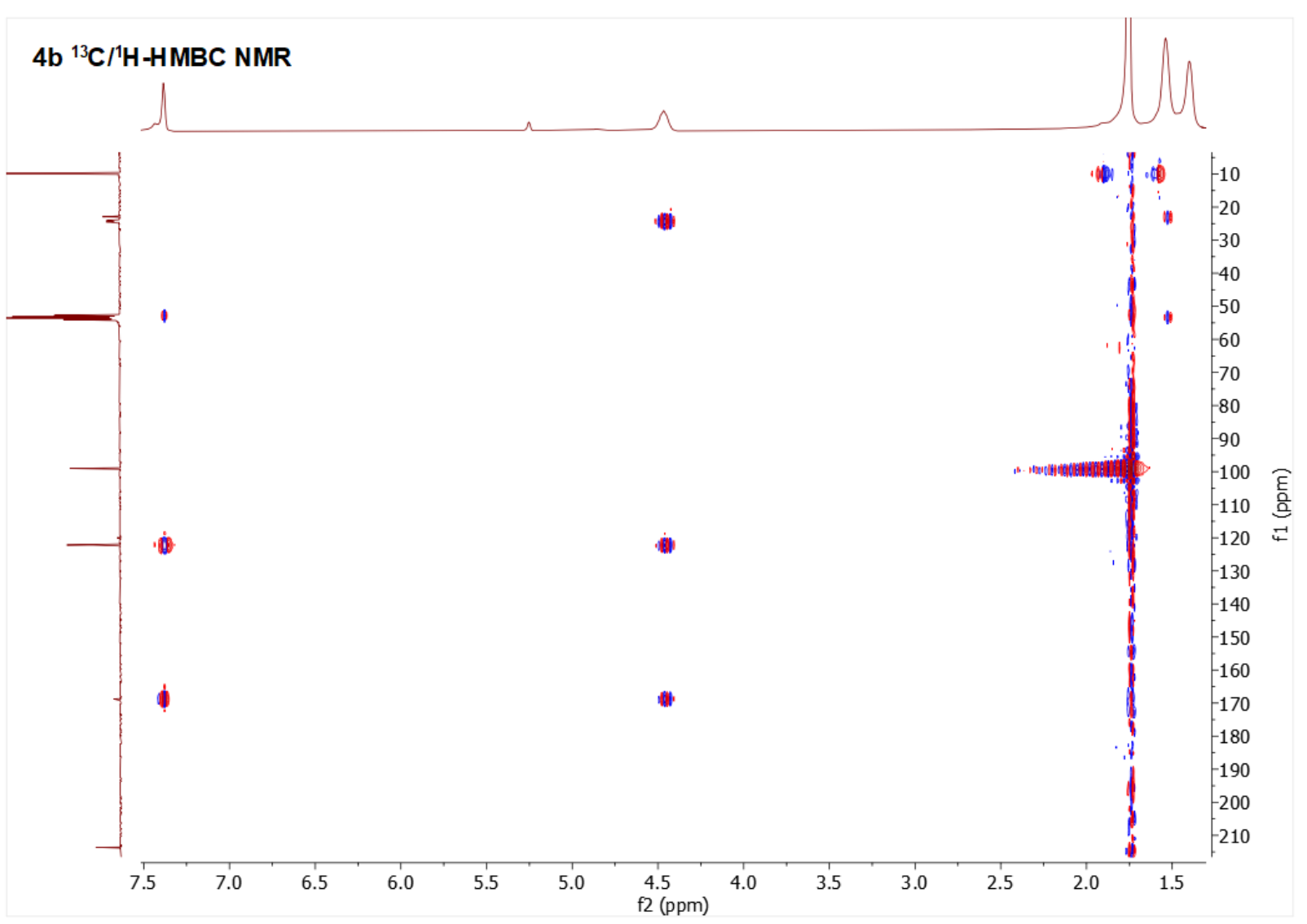

Figure S26. ${ }^{13} \mathrm{C} /{ }^{l} \mathrm{H}-\mathrm{HMBC} \mathrm{NMR}$ spectrum $\left(\mathrm{CD}_{2} \mathrm{Cl}_{2}\right)$ for complex $\mathbf{4 b}$. 


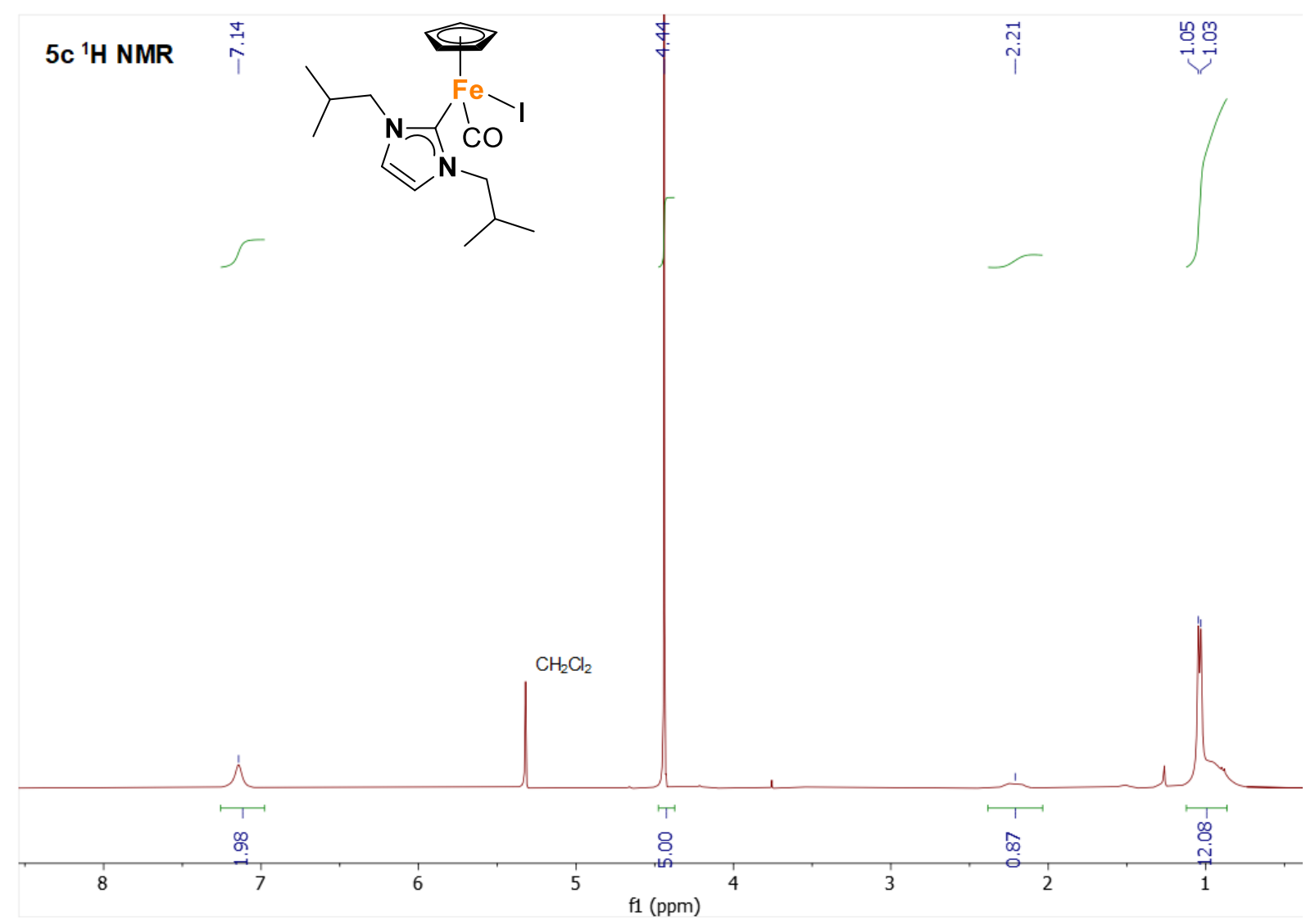

Figure S27. ${ }^{1} \mathrm{H} \mathrm{NMR}$ spectrum $\left(300 \mathrm{MHz}, \mathrm{CD}_{2} \mathrm{Cl}_{2}\right)$ for complex $\mathbf{5 c}$.

$5 c^{1}{ }^{1}$ NMR

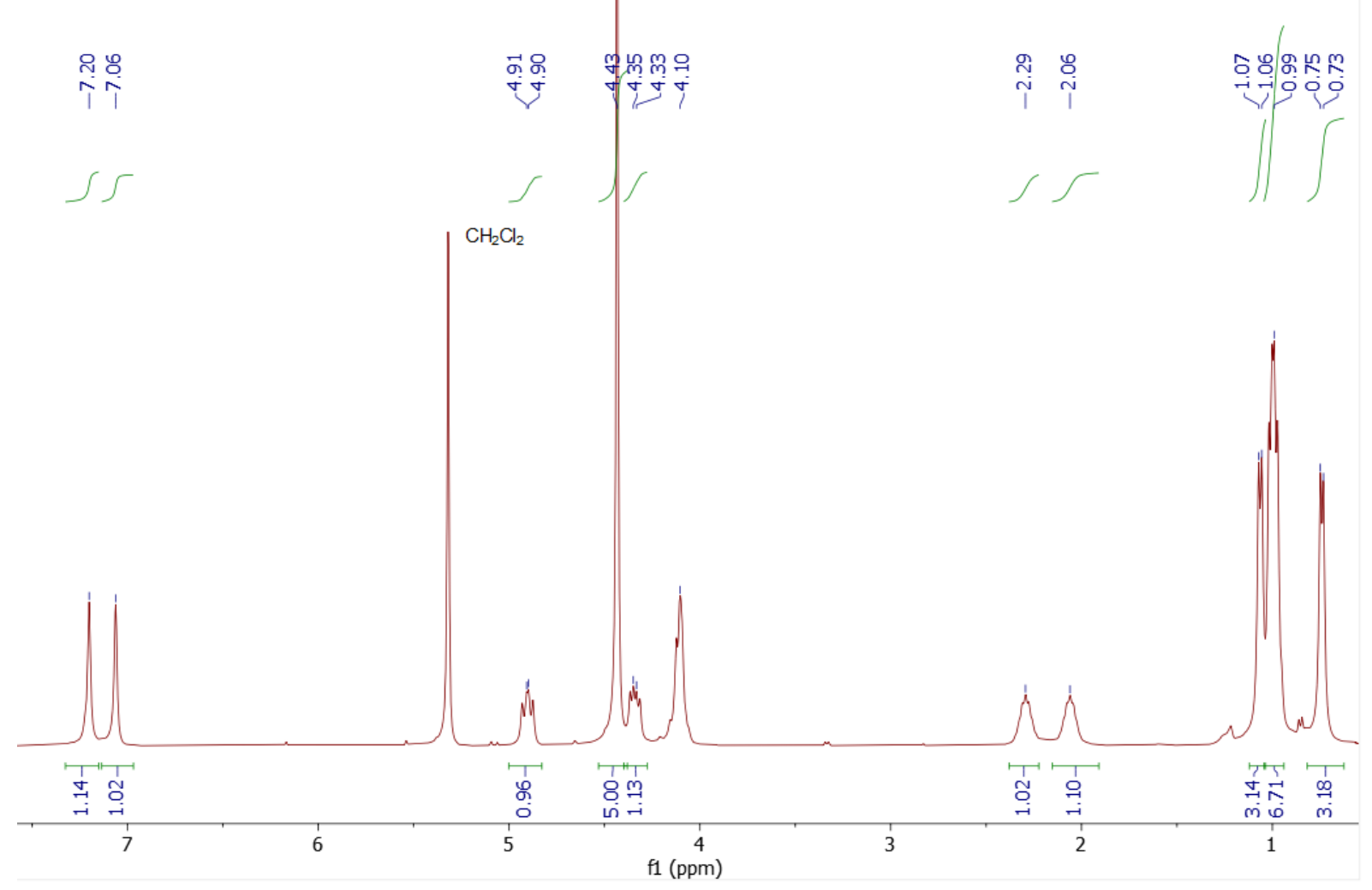

Figure S28. ${ }^{1} \mathrm{H} N M R$ spectrum $\left(300 \mathrm{MHz}, \mathrm{CD}_{2} \mathrm{Cl}_{2}\right)$ for complex $5 \mathrm{c}$ at $-20{ }^{\circ} \mathrm{C}$. 
$5{ }^{13} \mathrm{C}\left\{{ }^{1} \mathrm{H}\right\}$ NMR

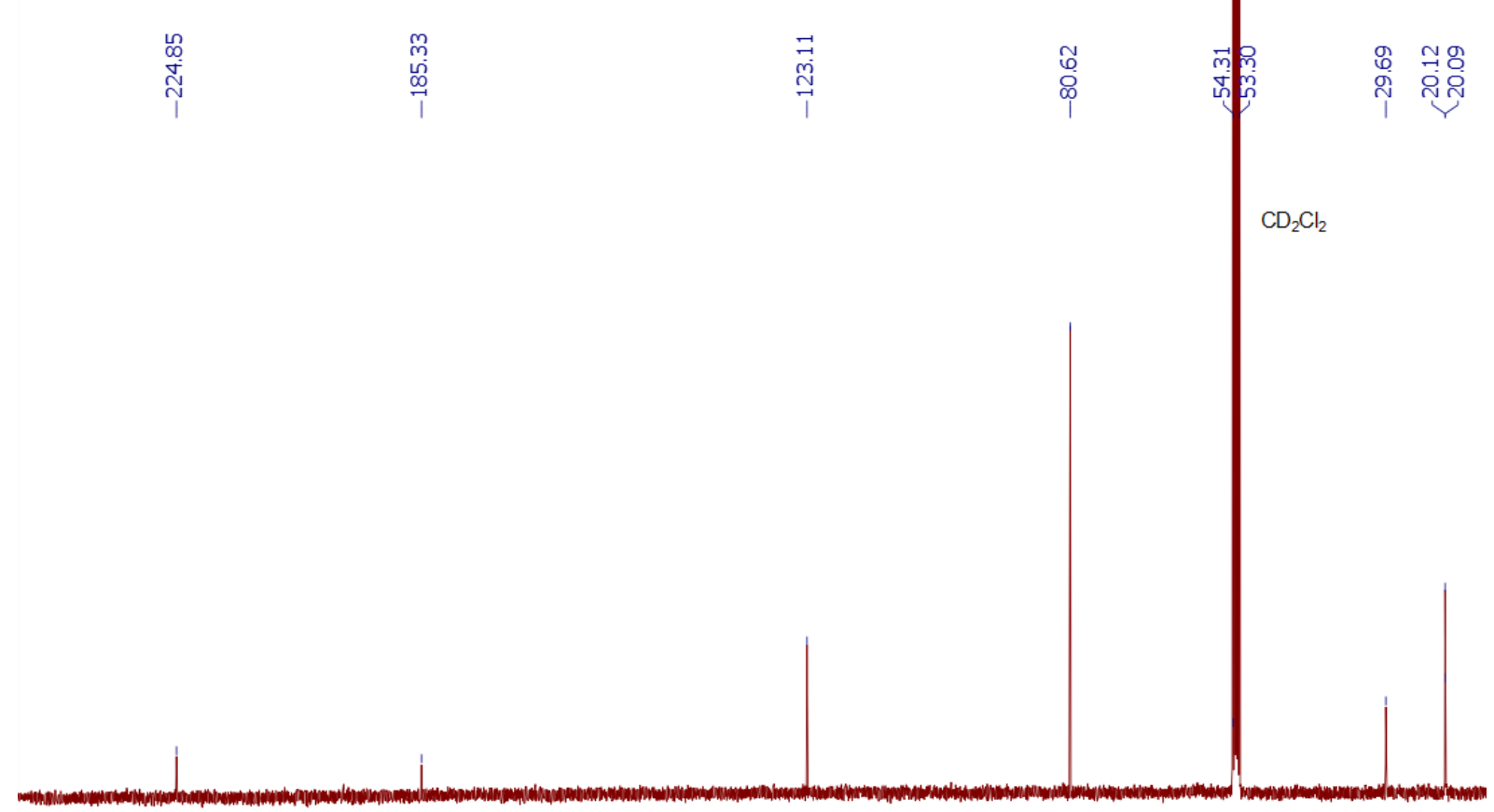

$240 \quad 200 \quad 180 \quad 160 \quad 140$ f1 (ppm)

Figure S29. $\left.{ }^{13} \mathrm{C}_{\{}{ }^{1} \mathrm{H}\right\} \mathrm{NMR}$ spectrum $\left(101 \mathrm{MHz}, \mathrm{CD}_{2} \mathrm{Cl}_{2}\right)$ for complex $\mathbf{5 c}$.

$5 \mathrm{C}^{13} \mathrm{C}\left\{{ }^{1} \mathrm{H}\right\} \mathrm{NMR}$

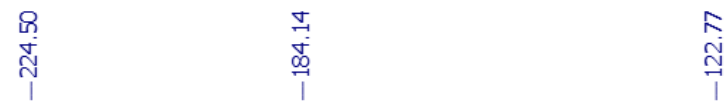

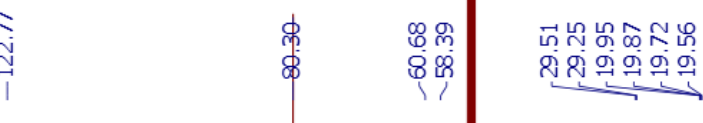

$\mathrm{CD}_{2} \mathrm{Cl}_{2}$

\begin{tabular}{|c|c|c|c|c|c|c|c|c|c|c|c|}
\hline 240 & 220 & 200 & 180 & 160 & $\begin{array}{l}140 \\
\mathrm{f} 1(\mathrm{ppm})\end{array}$ & 120 & 100 & 80 & 60 & 40 & 20 \\
\hline
\end{tabular}

Figure S30. ${ }^{13} \mathrm{C}_{\left\{{ }^{1} \mathrm{H}\right\}} \mathrm{NMR}$ spectrum $\left(101 \mathrm{MHz}, \mathrm{CD}_{2} \mathrm{Cl}_{2}\right)$ for complex $5 \boldsymbol{c}$ at $-20{ }^{\circ} \mathrm{C}$. 


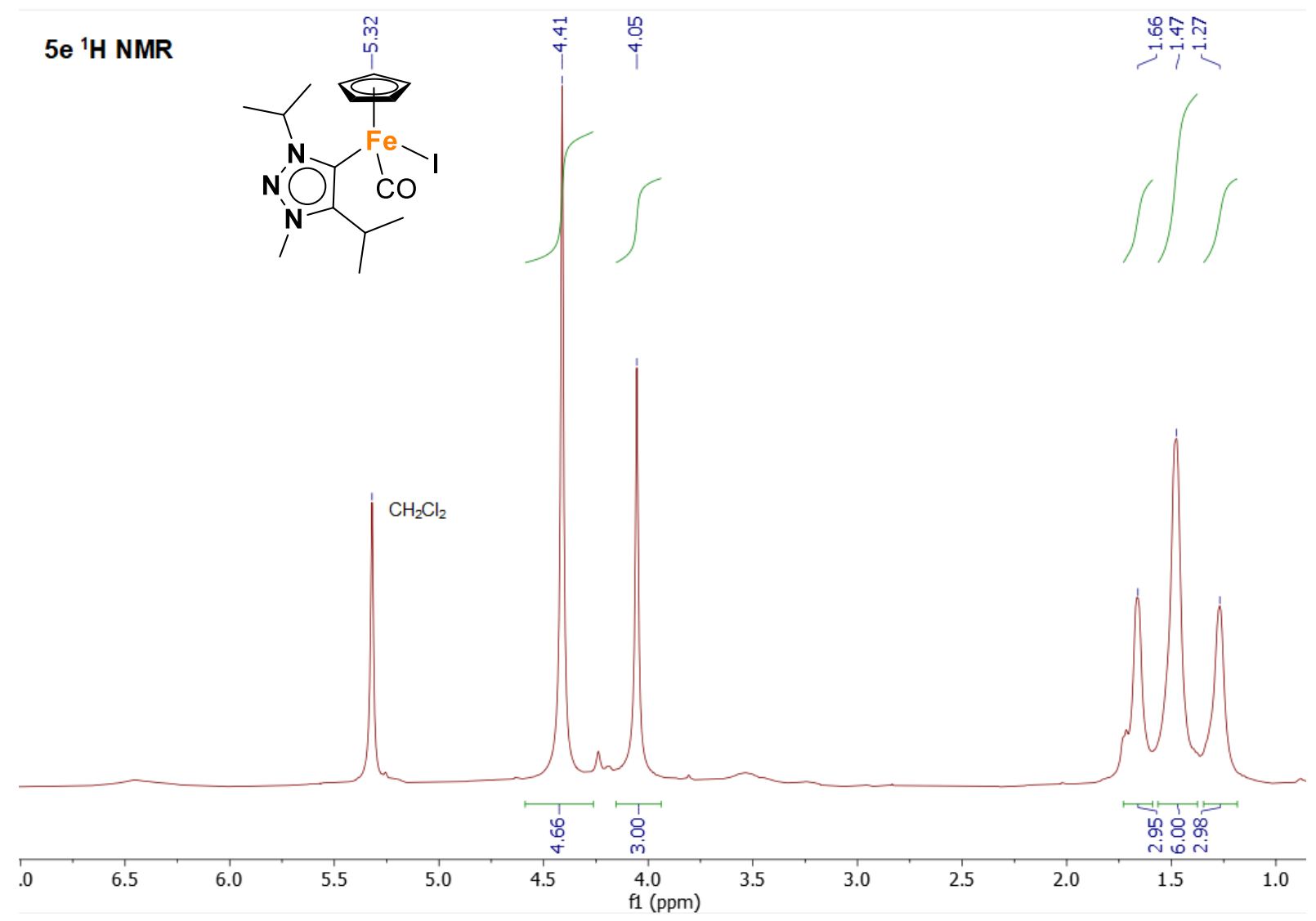

Figure S31. ${ }^{l} \mathrm{H} N M R$ spectrum $\left(300 \mathrm{MHz}, \mathrm{CD}_{2} \mathrm{Cl}_{2}\right)$ for complex $5 \boldsymbol{e}$ at $25{ }^{\circ} \mathrm{C}$

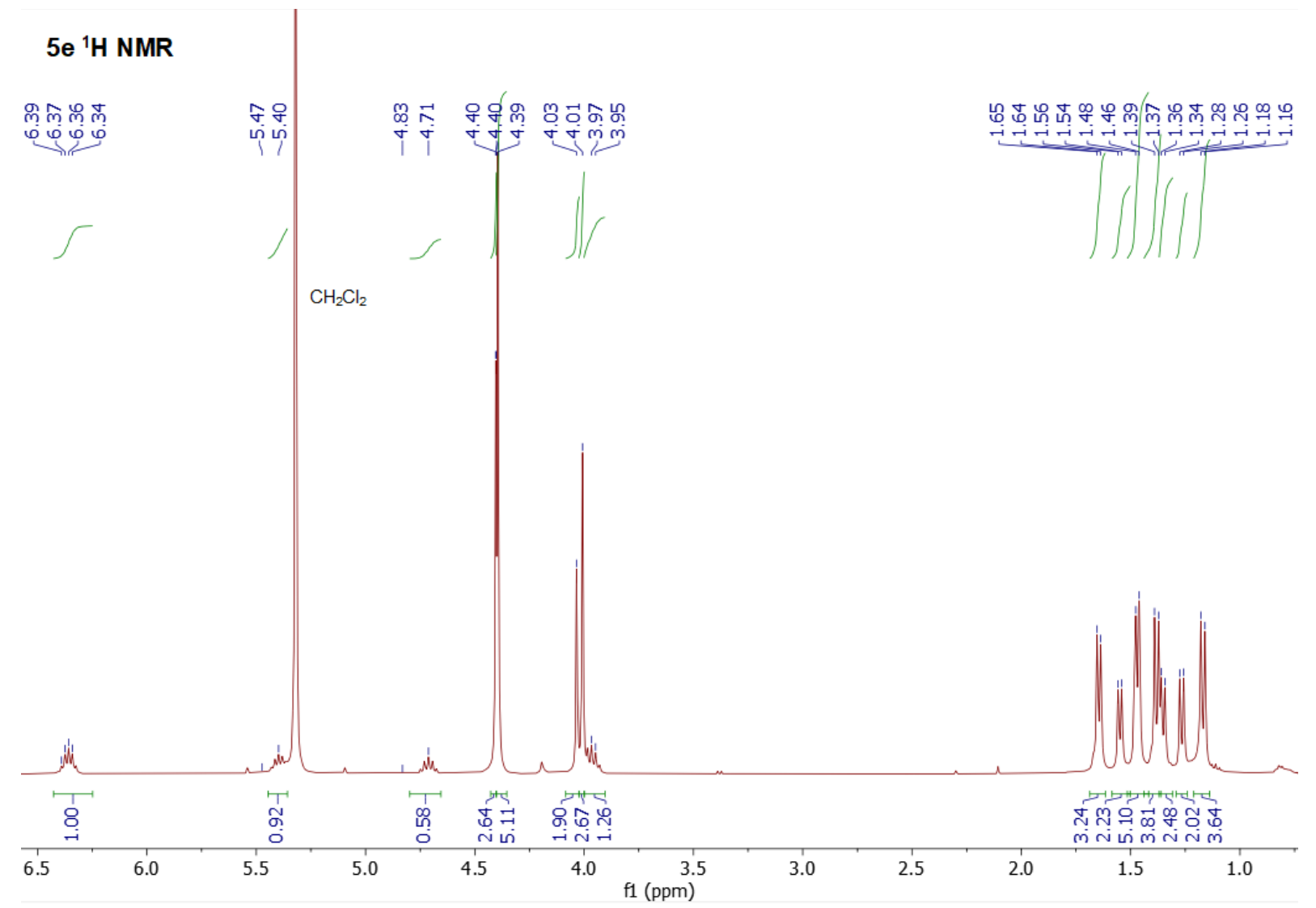

Figure S32. ${ }^{\mathrm{I}} \mathrm{H} \mathrm{NMR}$ spectrum $\left(400 \mathrm{MHz}, \mathrm{CD}_{2} \mathrm{Cl}_{2}\right)$ for complex 5 e at $-25{ }^{\circ} \mathrm{C}$ 


\begin{tabular}{|c|c|c|c|c|c|c|c|c|c|c|c|c|}
\hline 240 & 220 & 200 & 180 & 160 & 140 & $\begin{array}{l}120 \\
\mathrm{f} 1(\mathrm{ppm})\end{array}$ & 100 & 80 & 60 & 40 & 20 & 0 \\
\hline
\end{tabular}

Figure S33. ${ }^{13} \mathrm{C}_{\left\{{ }^{1} \mathrm{H}\right\}} \mathrm{NMR}$ spectrum $\left(101 \mathrm{MHz}, \mathrm{CD}_{2} \mathrm{Cl}_{2}\right)$ for complex $5 \boldsymbol{e}$ at $25{ }^{\circ} \mathrm{C}$ $5 e^{13} \mathrm{C}\left\{{ }^{1} \mathrm{H}\right\}$ NMR

峹
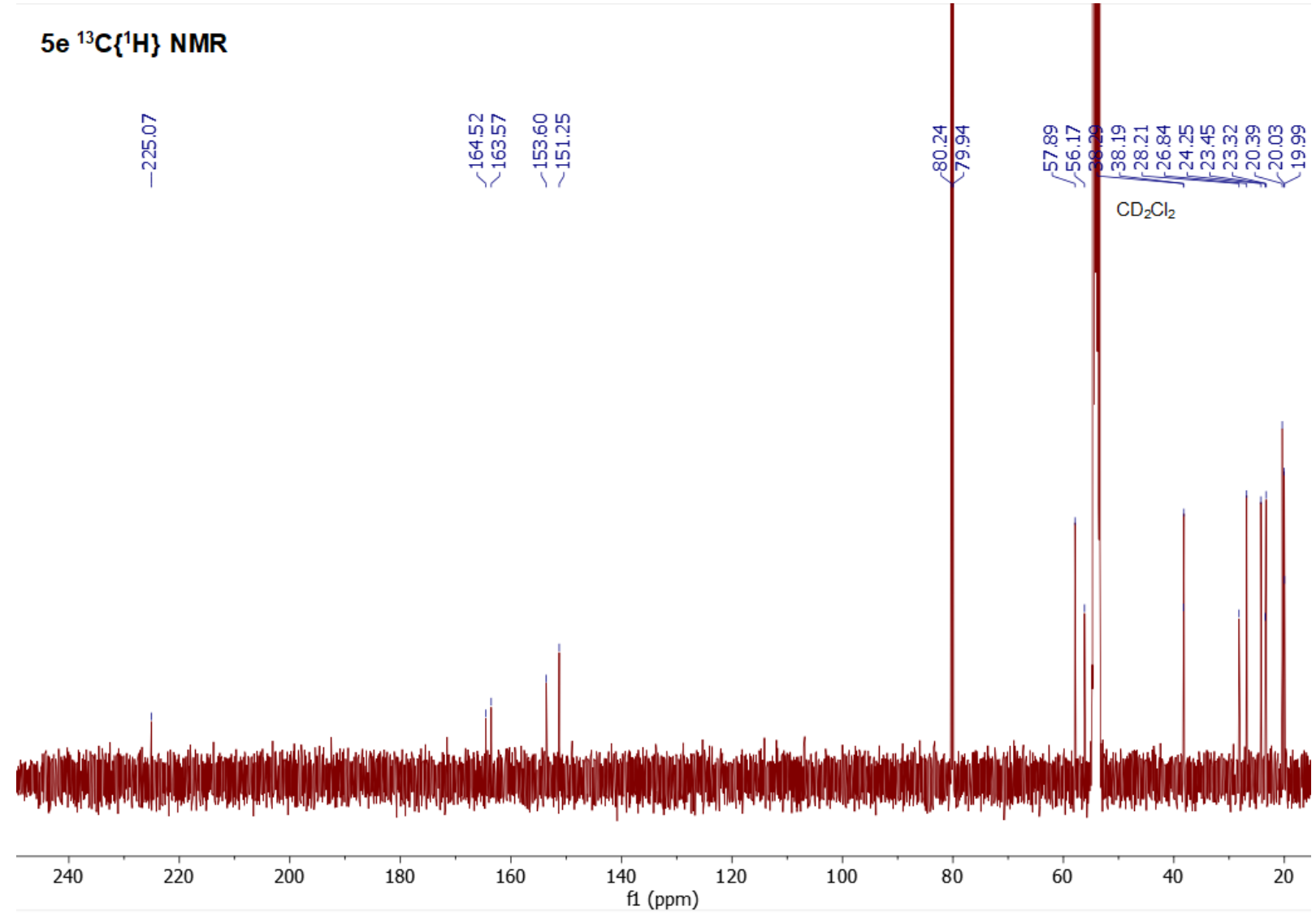

Figure S34. $\left.{ }^{13} C_{\{}^{\{1} \mathrm{H}\right\}$ NMR spectrum $\left(101 \mathrm{MHz}, \mathrm{CD}_{2} \mathrm{Cl}_{2}\right)$ for complex $5 \boldsymbol{e}$ at $-25{ }^{\circ} \mathrm{C}$ 
$5 \mathrm{e}^{13} \mathrm{C} /{ }^{1} \mathrm{H}-\mathrm{HMBC}$ NMR

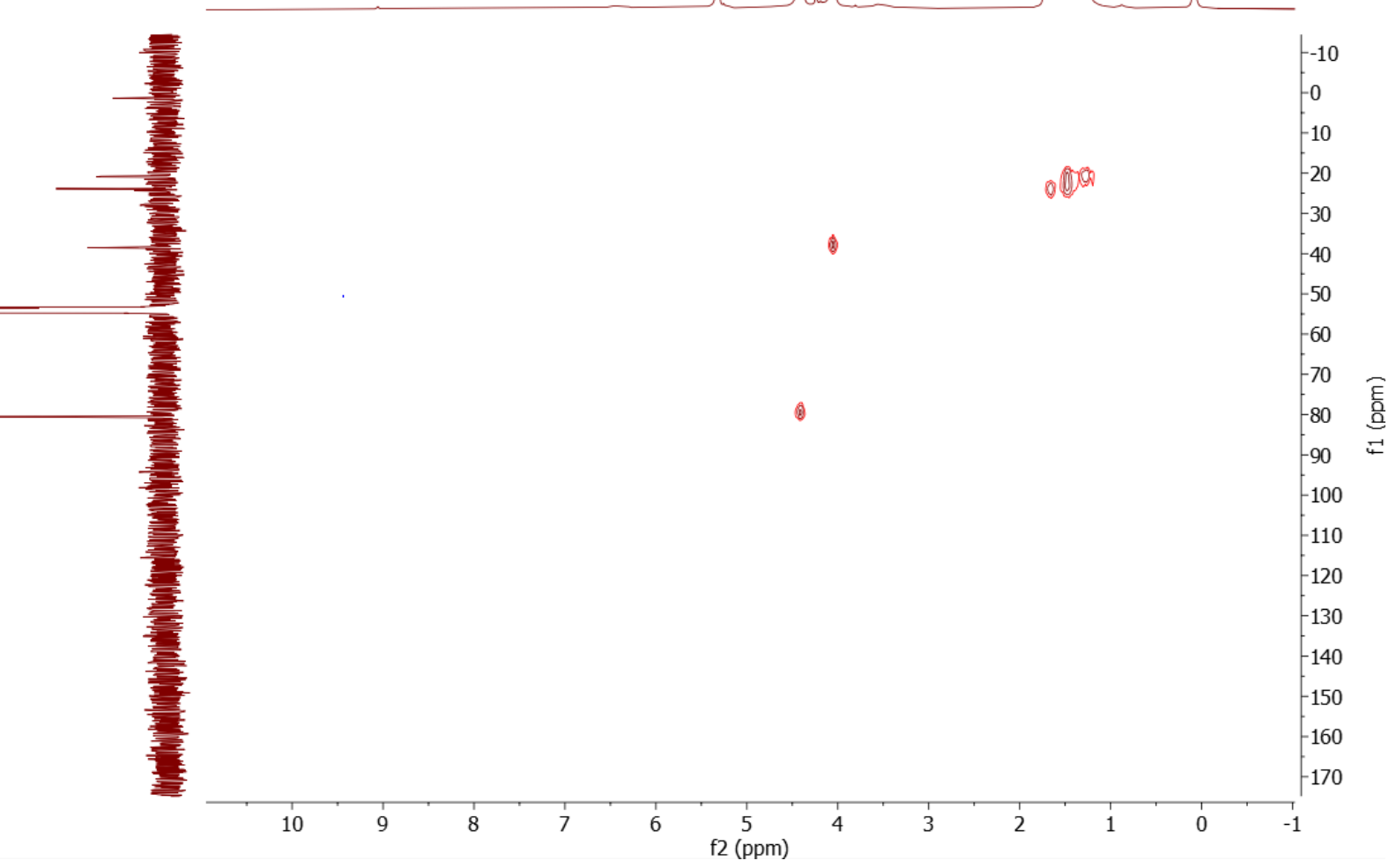

Figure S35. ${ }^{13} \mathrm{C} /{ }^{l} \mathrm{H}-\mathrm{HMBC}$ NMR spectrum $\left(\mathrm{CD}_{2} \mathrm{Cl}_{2}\right)$ for complex 5 e at $25{ }^{\circ} \mathrm{C}$

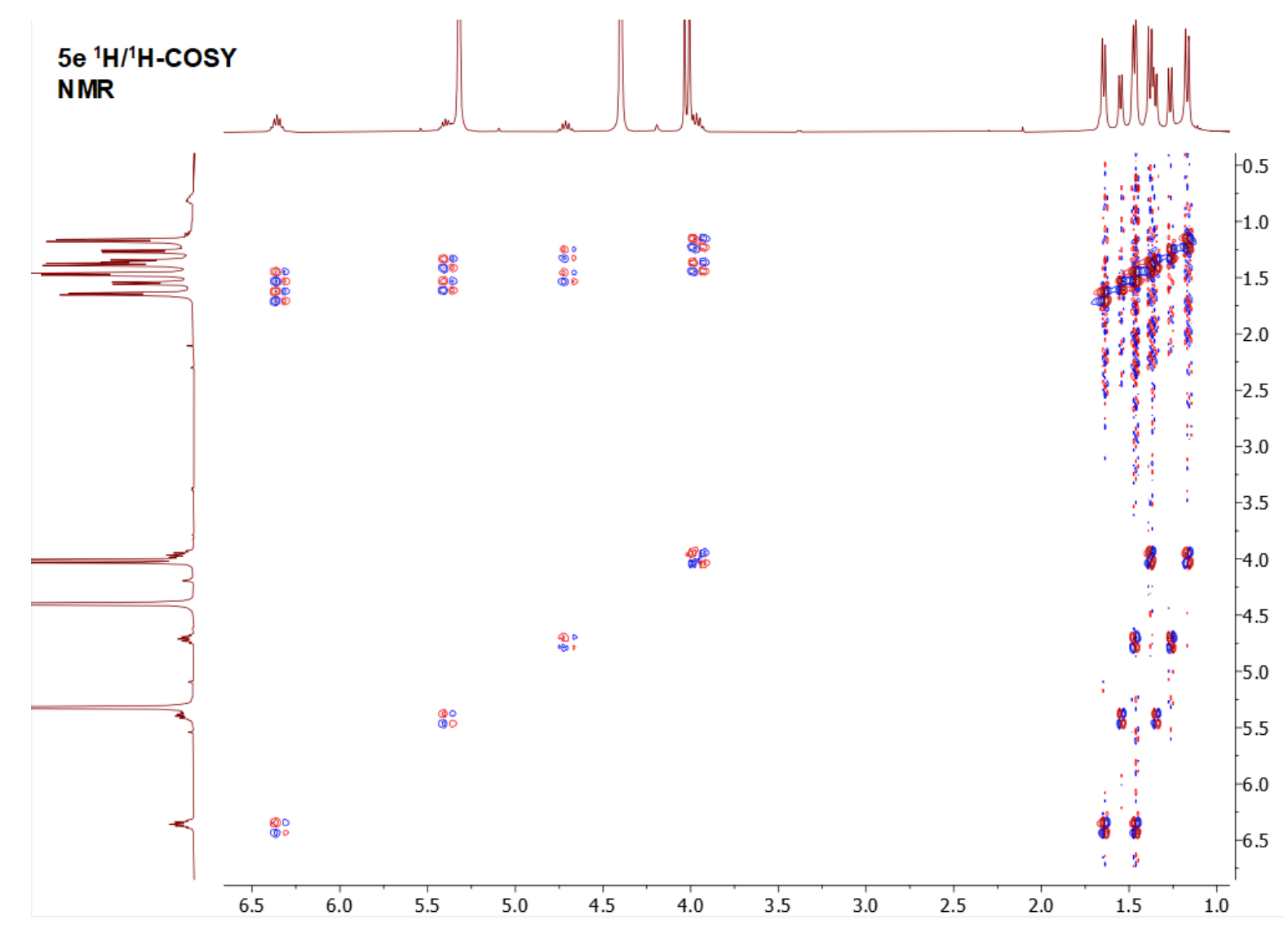

Figure S36. ${ }^{l} \mathrm{H} /{ }^{l} \mathrm{H}$-COSY NMR spectrum $\left(\mathrm{CD}_{2} \mathrm{Cl}_{2}\right)$ for complex $5 \boldsymbol{e}$ at $-25{ }^{\circ} \mathrm{C}$. 


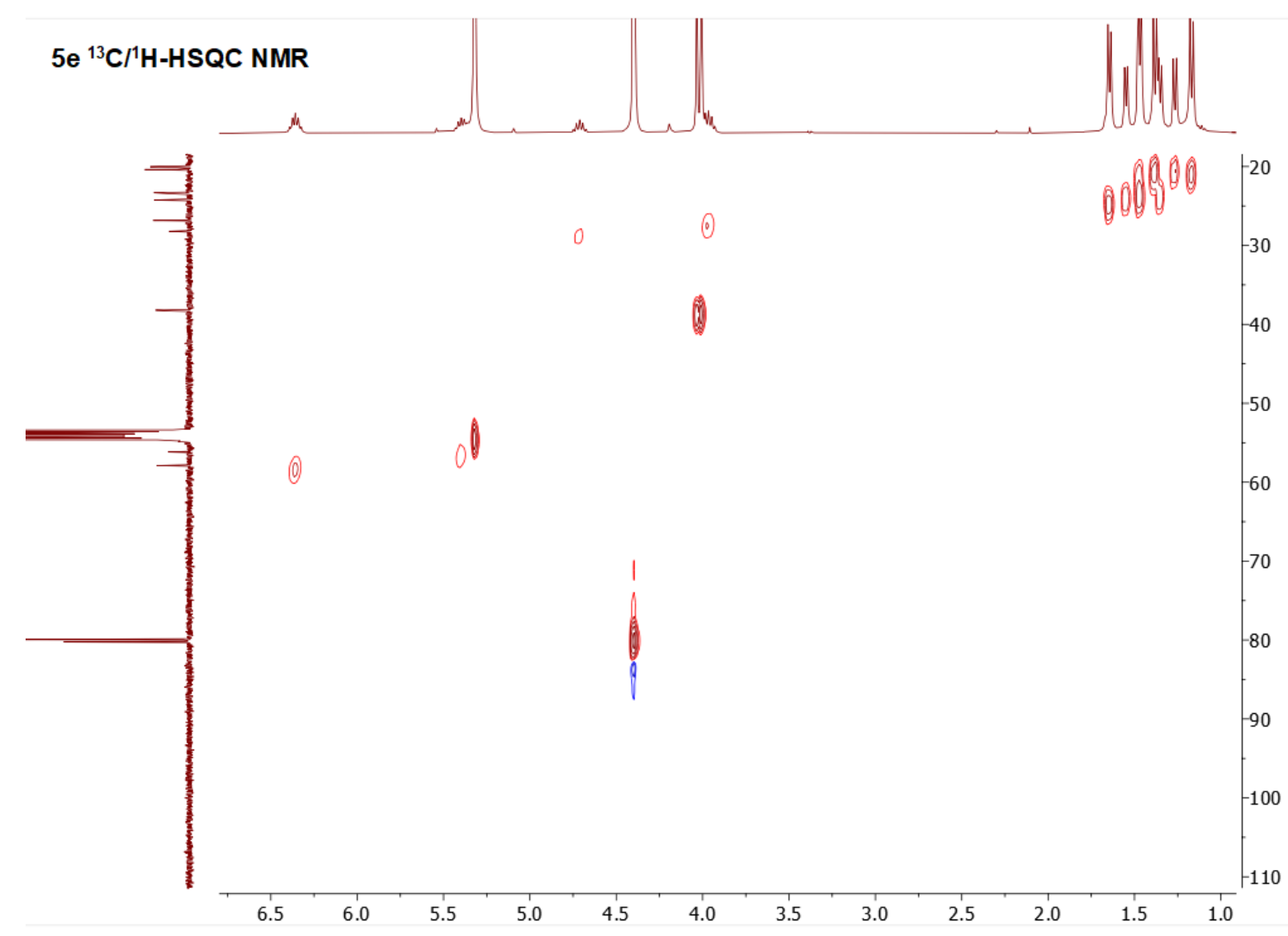

Figure S37. ${ }^{13} \mathrm{C} /{ }^{1} \mathrm{H}-\mathrm{HSQC}$ NMR spectrum $\left(\mathrm{CD}_{2} \mathrm{Cl}_{2}\right)$ for complex $\mathbf{5 e}$ at $-25{ }^{\circ} \mathrm{C}$.

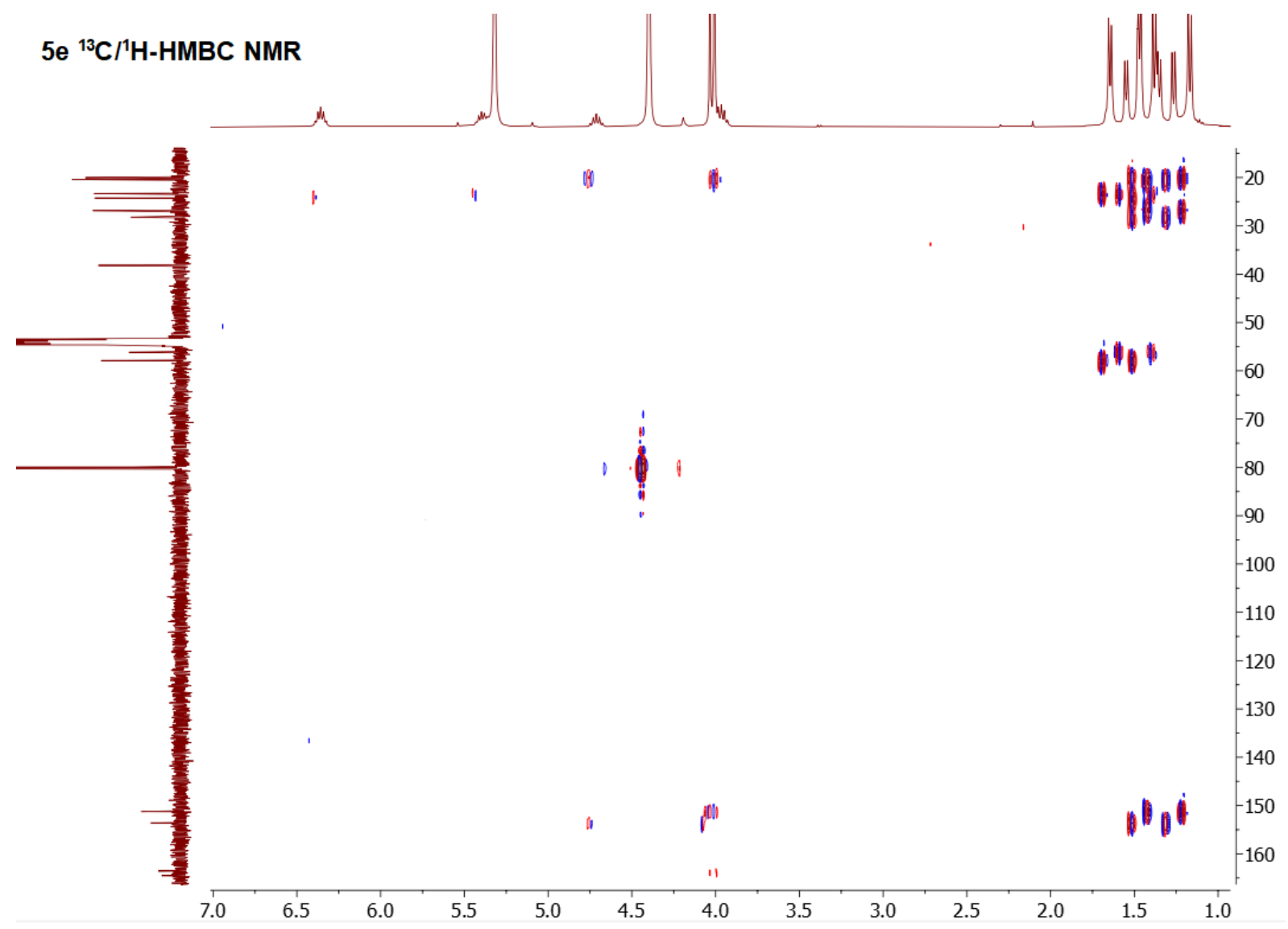

Figure S38. ${ }^{13} \mathrm{C} / \mathrm{H}-\mathrm{HMBC}$ NMR spectrum $\left(\mathrm{CD}_{2} \mathrm{Cl}_{2}\right)$ for complex 5 e at $-25{ }^{\circ} \mathrm{C}$ 


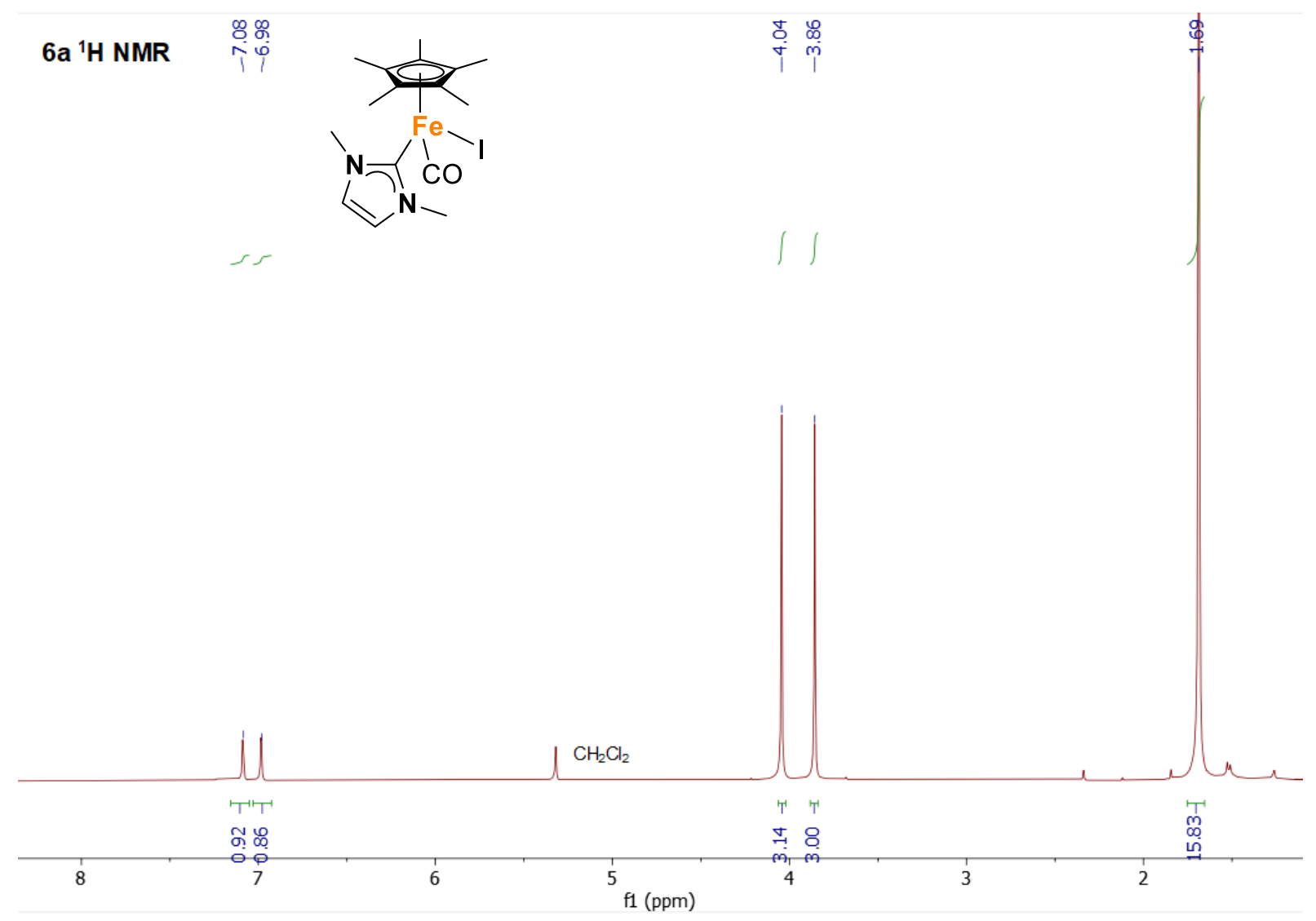

Figure S39. ${ }^{1} \mathrm{H} N M R$ spectrum $\left(400 \mathrm{MHz}, \mathrm{CD}_{2} \mathrm{Cl}_{2}\right)$ for complex $\boldsymbol{6}$.

\section{$6 a{ }^{13} \mathrm{C}\left\{{ }^{1} \mathrm{H}\right\}$ NMR}

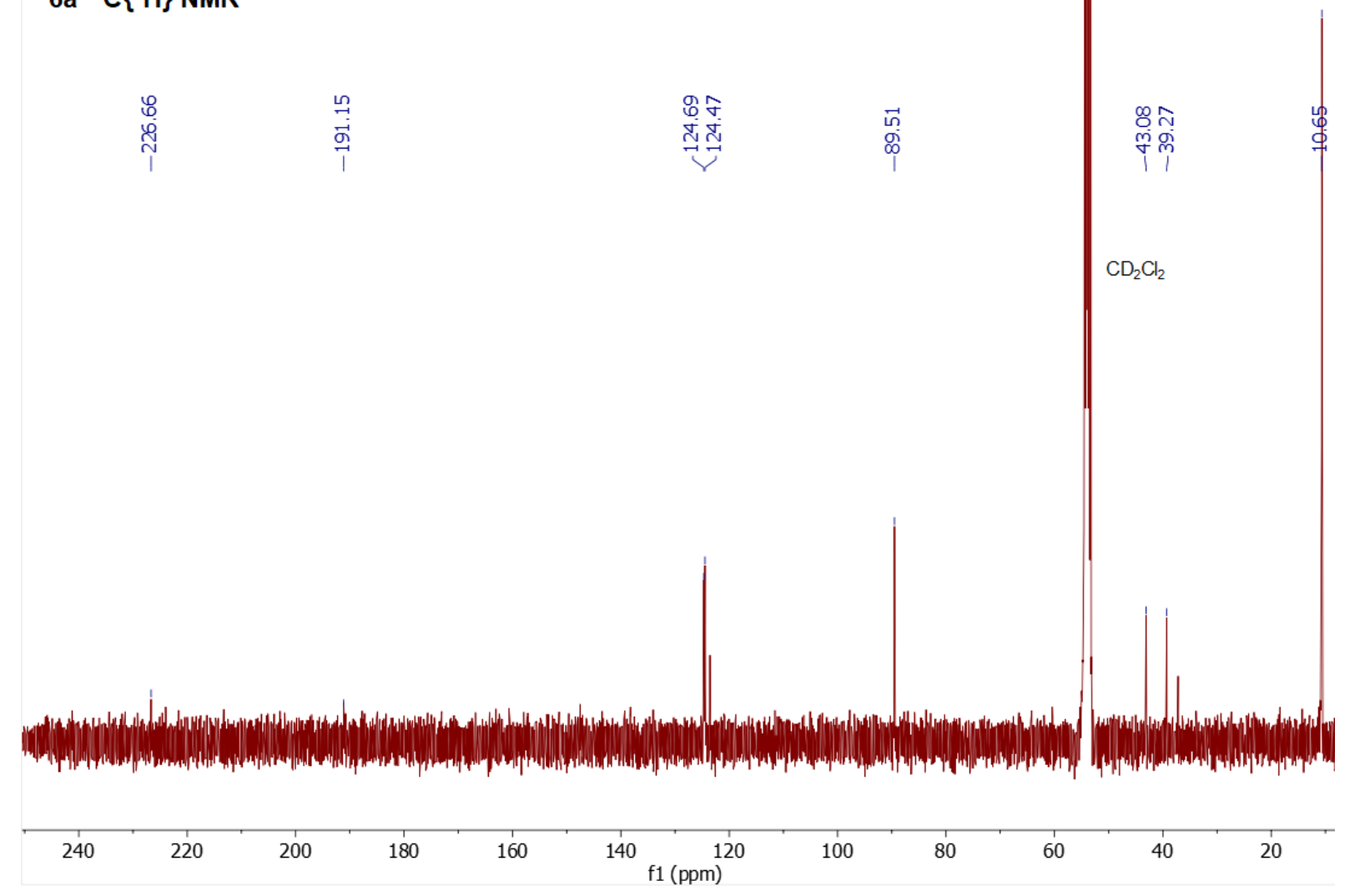

Figure S40. $\left.{ }^{13} C_{\{}^{\{1} H\right\}$ NMR spectrum $\left(101 \mathrm{MHz}, \mathrm{CD}_{2} \mathrm{Cl}_{2}\right)$ for complex $\boldsymbol{6} \boldsymbol{a}$. 


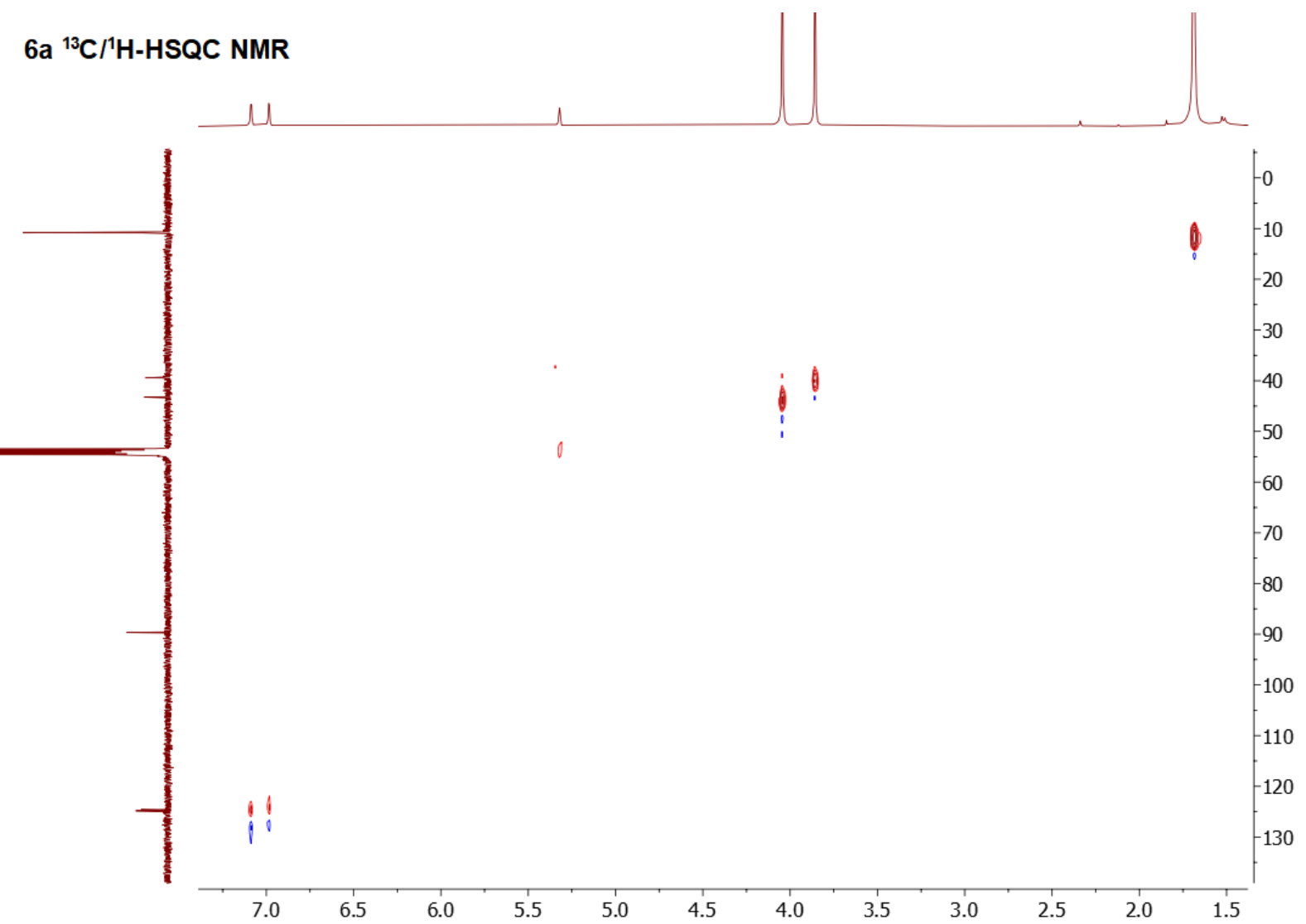

Figure $\mathbf{S 4 1 .}{ }^{13} \mathrm{C} /{ }^{1} \mathrm{H}-\mathrm{HSQC}$ NMR spectrum $\left(\mathrm{CD}_{2} \mathrm{Cl}_{2}\right)$ for complex $\boldsymbol{6}$.

$6 a{ }^{13} \mathrm{C} /{ }^{1} \mathrm{H}-\mathrm{HMBC}$ NMR

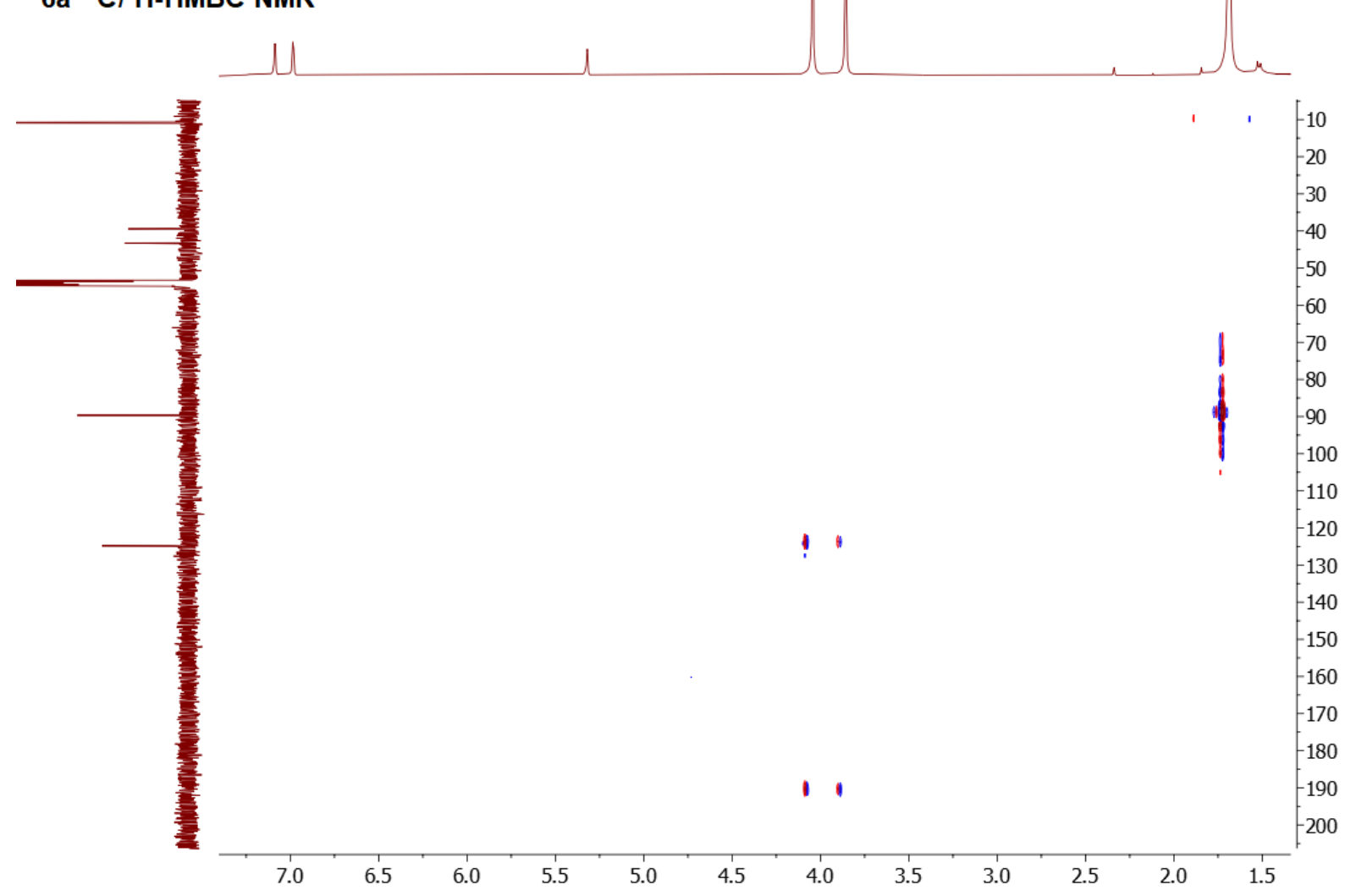

Figure $\mathbf{S 4 2 .}{ }^{13} \mathrm{C} /{ }^{1} \mathrm{H}-\mathrm{HMBC}$ NMR spectrum $\left(\mathrm{CD}_{2} \mathrm{Cl}_{2}\right)$ for complex $\boldsymbol{6} \boldsymbol{a}$. 
6b ${ }^{1} \mathrm{H}$ NMR

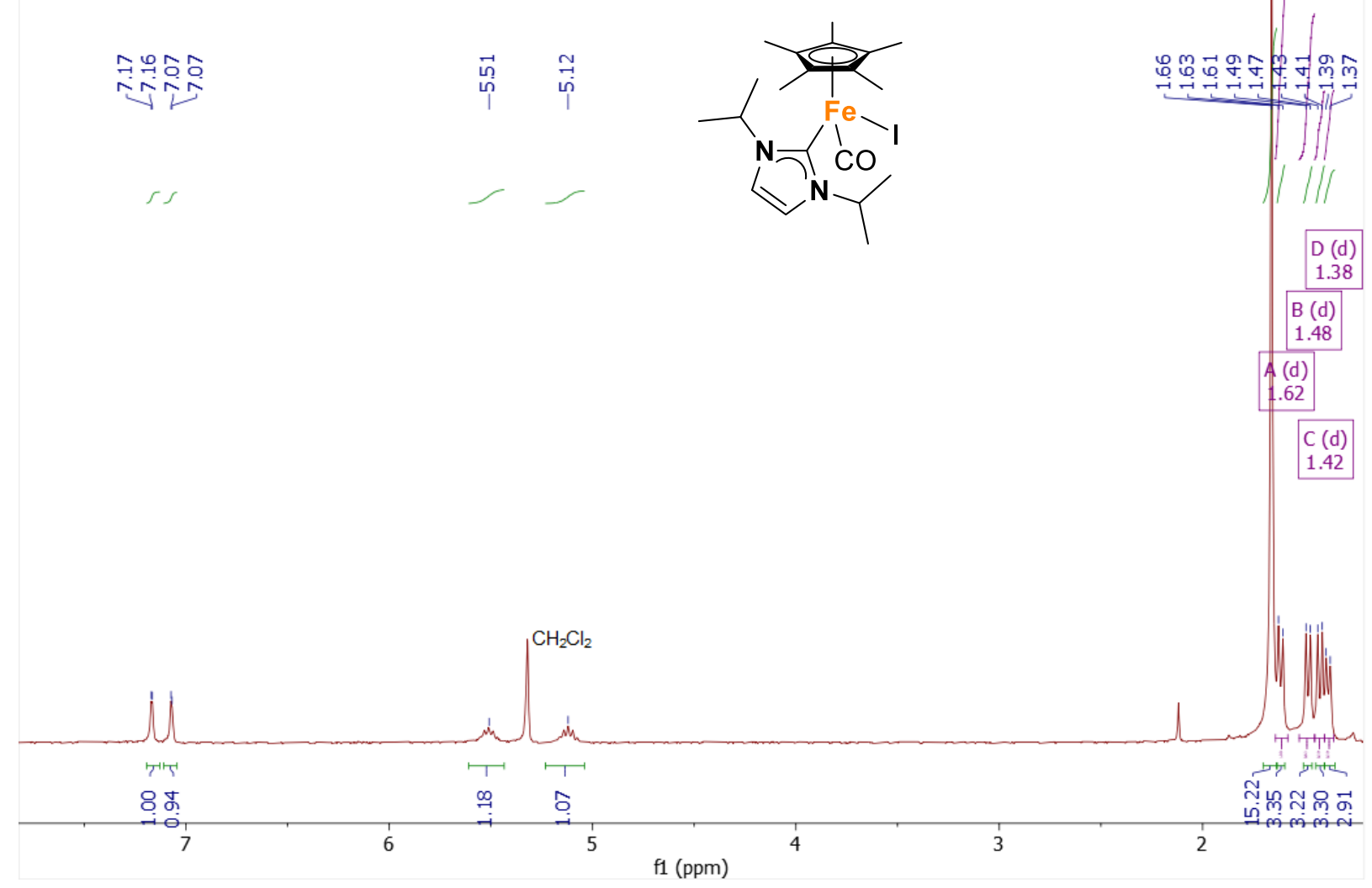

Figure S43. ${ }^{\mathrm{I}} \mathrm{H} \mathrm{NMR}$ spectrum (300 $\mathrm{MHz}, \mathrm{CD}_{2} \mathrm{Cl}_{2}$ ) for complex $\boldsymbol{6 b}$.

$6 b{ }^{13} \mathrm{C}\left\{{ }^{1} \mathrm{H}\right\}$ NMR

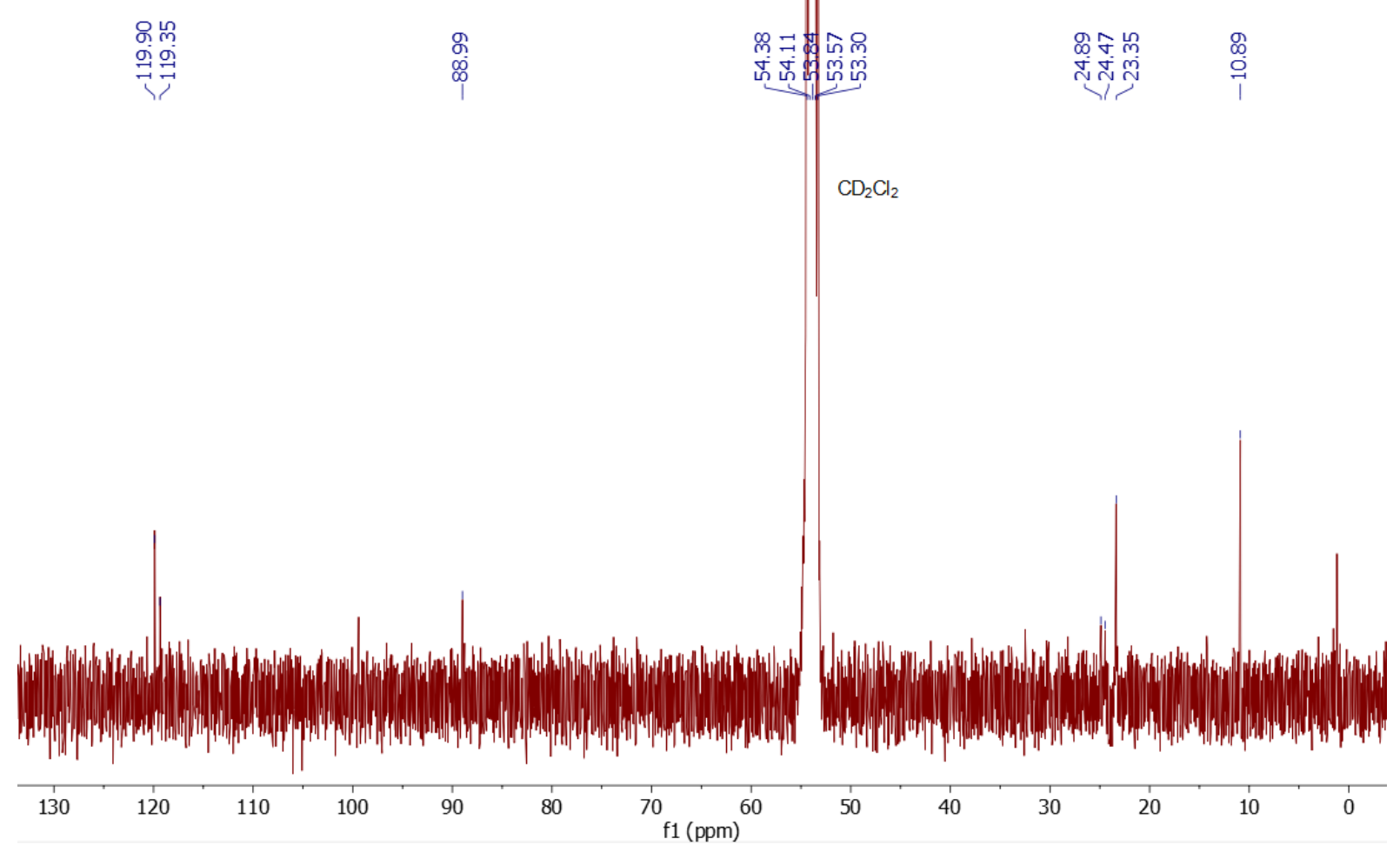

Figure S44. $\left.{ }^{13} C_{\{}{ }^{1} H\right\}$ NMR spectrum (101 $\left.\mathrm{MHz}, \mathrm{CD}_{2} \mathrm{Cl}_{2}\right)$ for complex $\boldsymbol{6} \boldsymbol{b}$. 


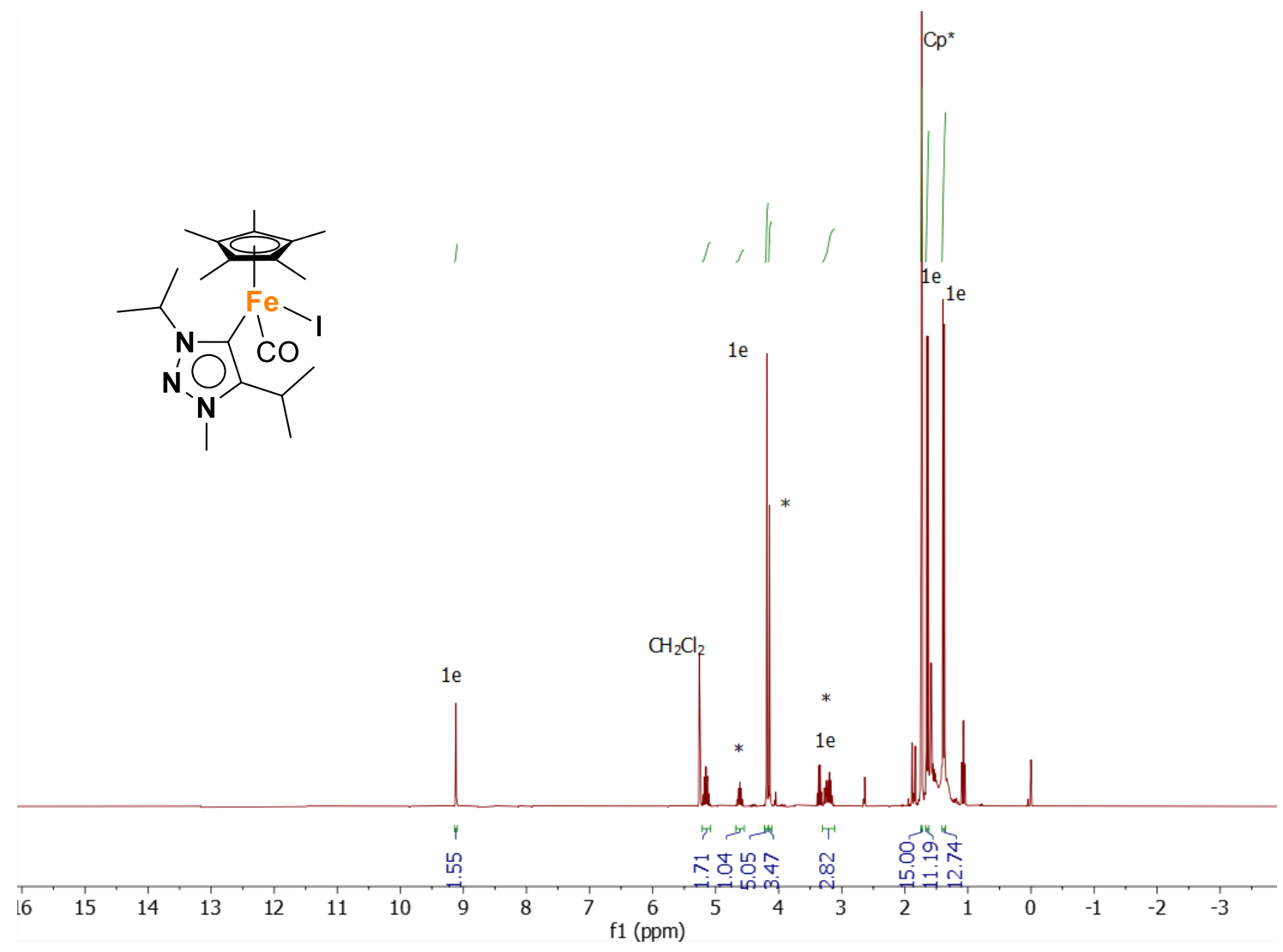

Figure S45. ${ }^{1} \mathrm{H} \mathrm{NMR}$ spectrum $\left(300 \mathrm{MHz}, \mathrm{CD}_{2} \mathrm{Cl}_{2}\right)$ for proposed complex ligand signals(le) proposed complex $(*)$.

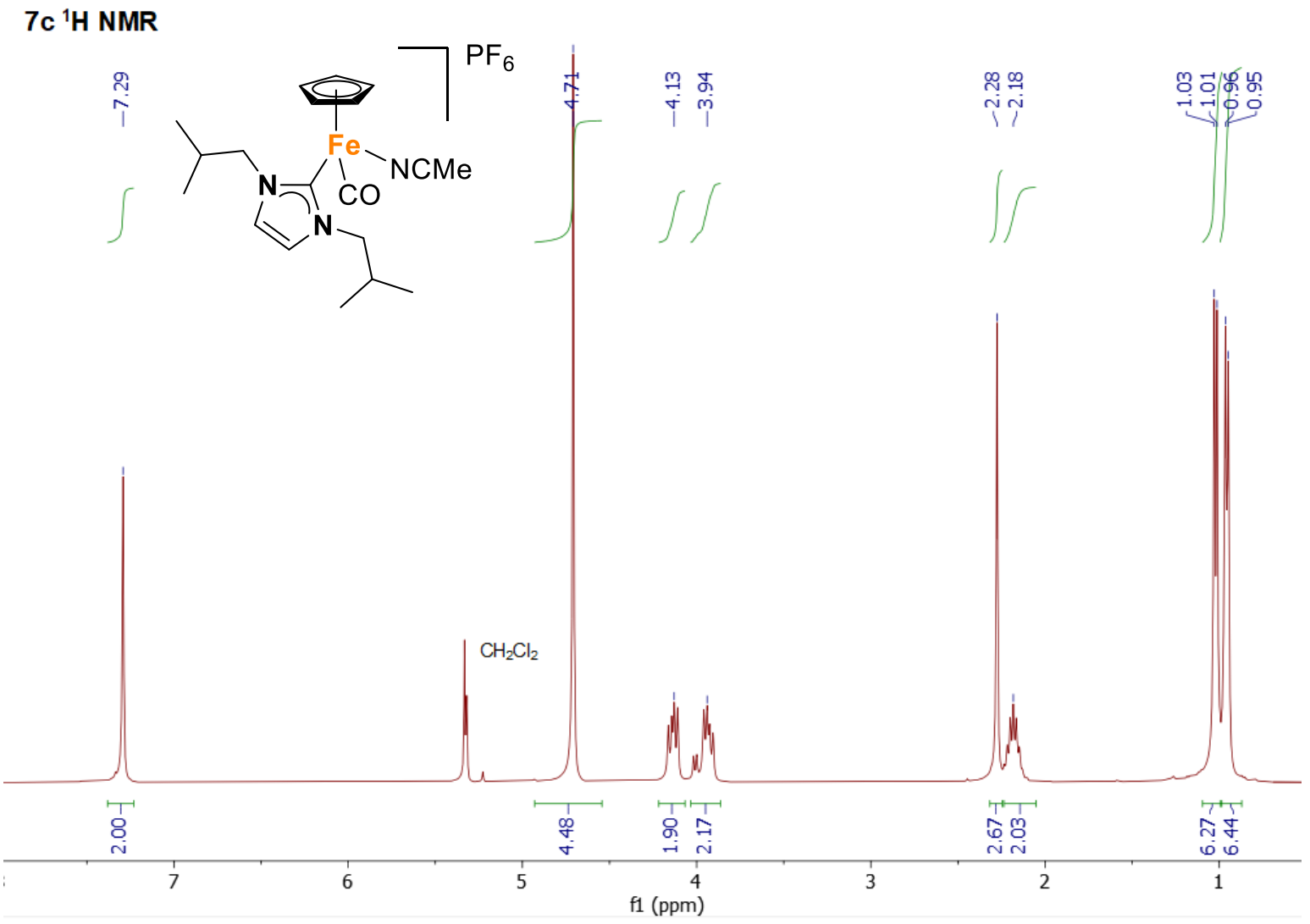

Figure S46. ${ }^{1} \mathrm{H} N \mathrm{MR}$ spectrum $\left(300 \mathrm{MHz}, \mathrm{CD}_{2} \mathrm{Cl}_{2}\right)$ for complex $7 \mathrm{c}$. 
7c ${ }^{13} \mathrm{C}\left\{{ }^{1} \mathrm{H}\right\}$ NMR

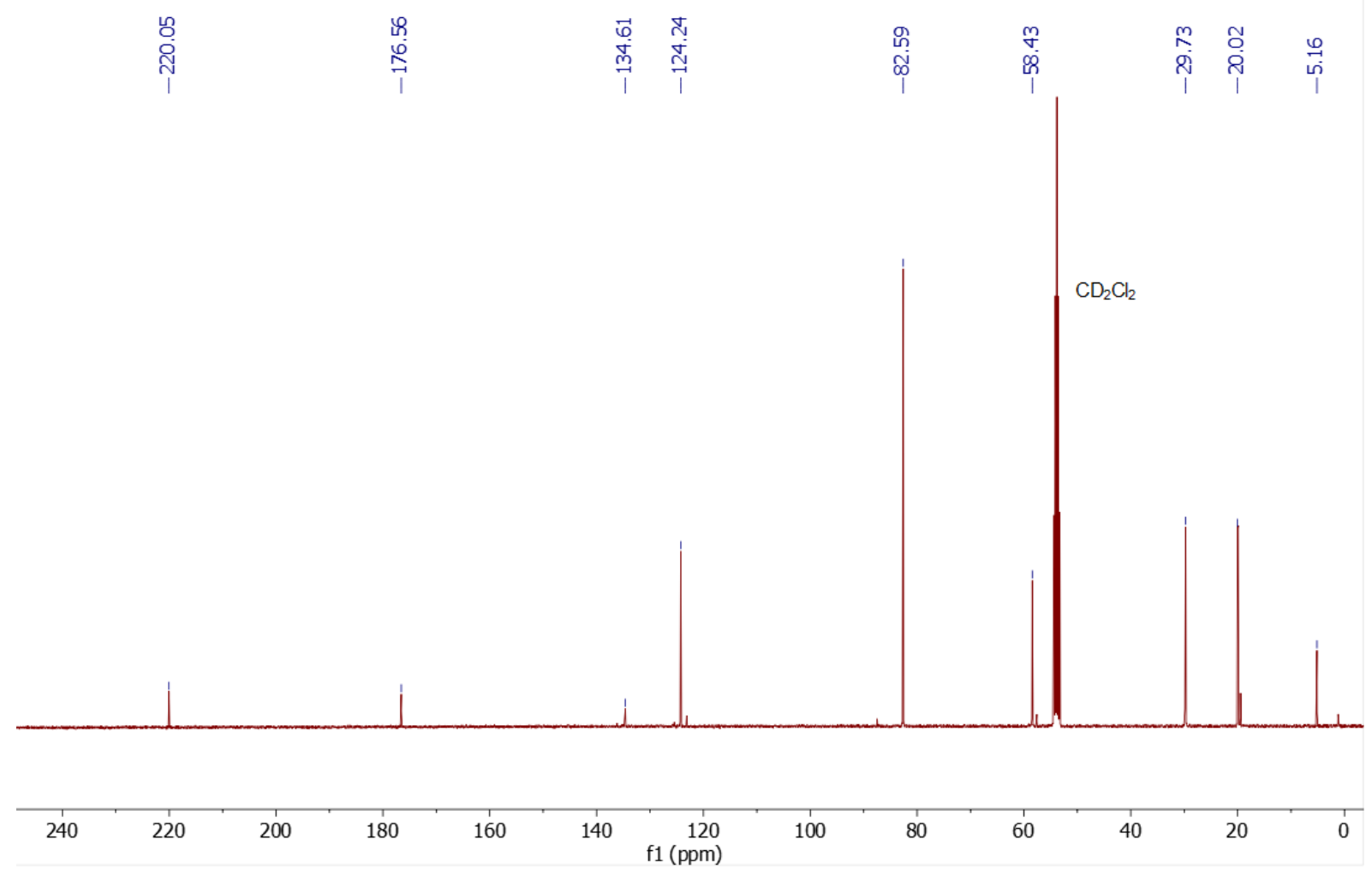

Figure S47. $\left.{ }^{13} C_{\{}{ }^{1} \mathrm{H}\right\}$ NMR spectrum $\left(101 \mathrm{MHz}, \mathrm{CD}_{2} \mathrm{Cl}_{2}\right)$ for complex $7 \boldsymbol{c}$.

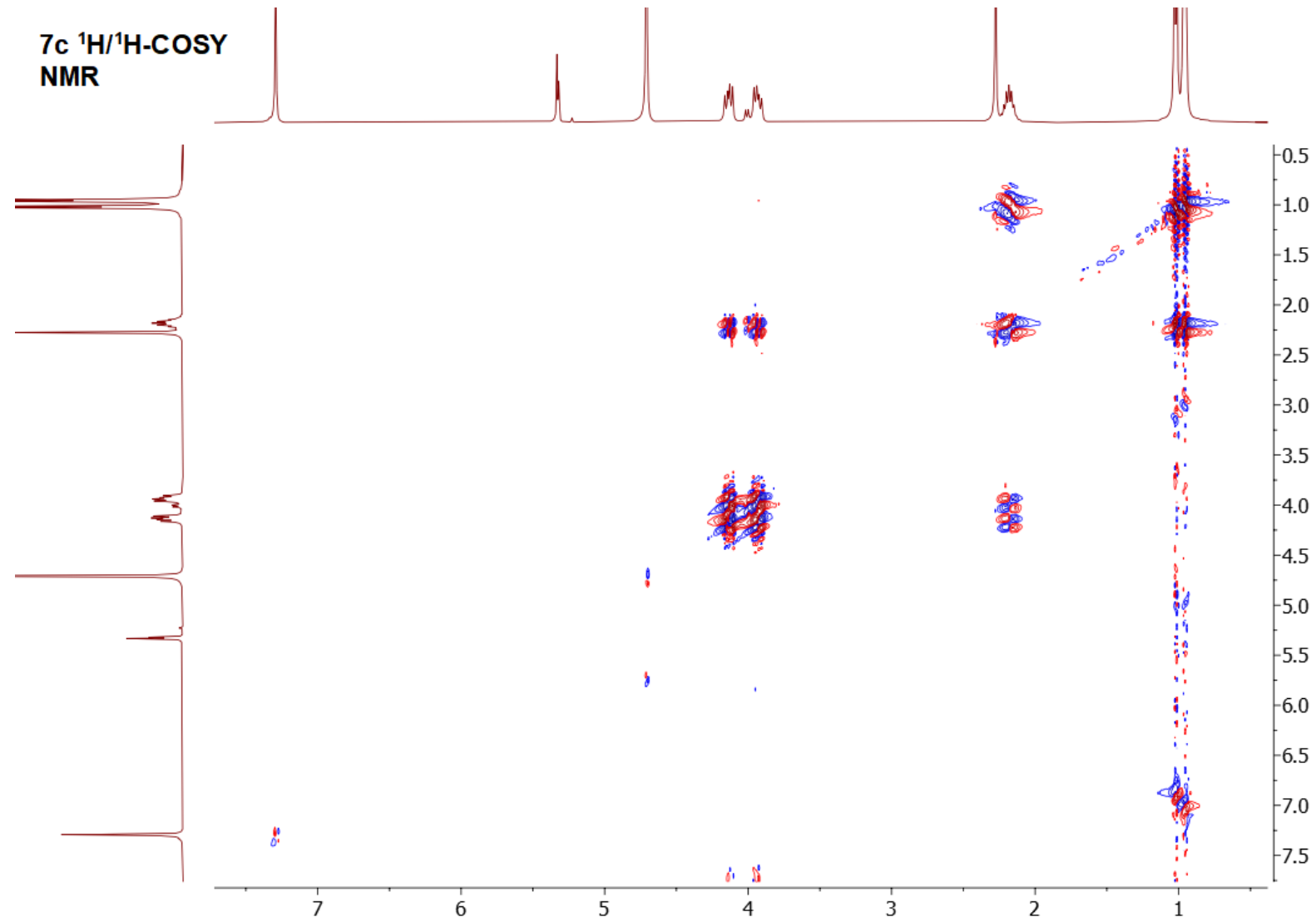

Figure S48. ${ }^{1} \mathrm{H} /{ }^{1} \mathrm{H}$-COSY NMR spectrum $\left(\mathrm{CD}_{2} \mathrm{Cl}_{2}\right)$ for complex $7 \boldsymbol{c}$. 


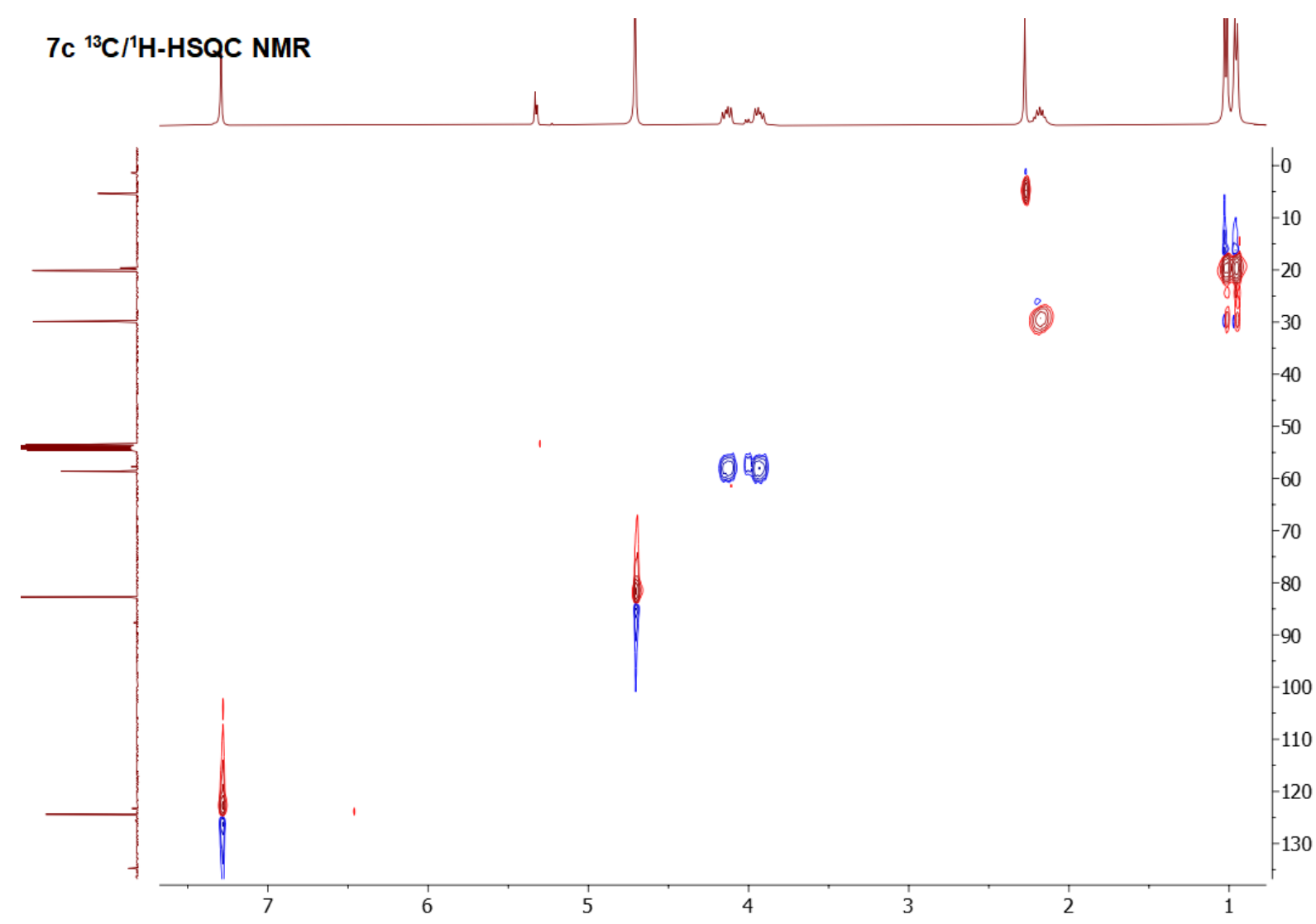

Figure S49. ${ }^{13} \mathrm{C} /{ }^{1} \mathrm{H}-\mathrm{HSQC}$ NMR spectrum $\left(\mathrm{CD}_{2} \mathrm{Cl}_{2}\right)$ for complex 7 c.

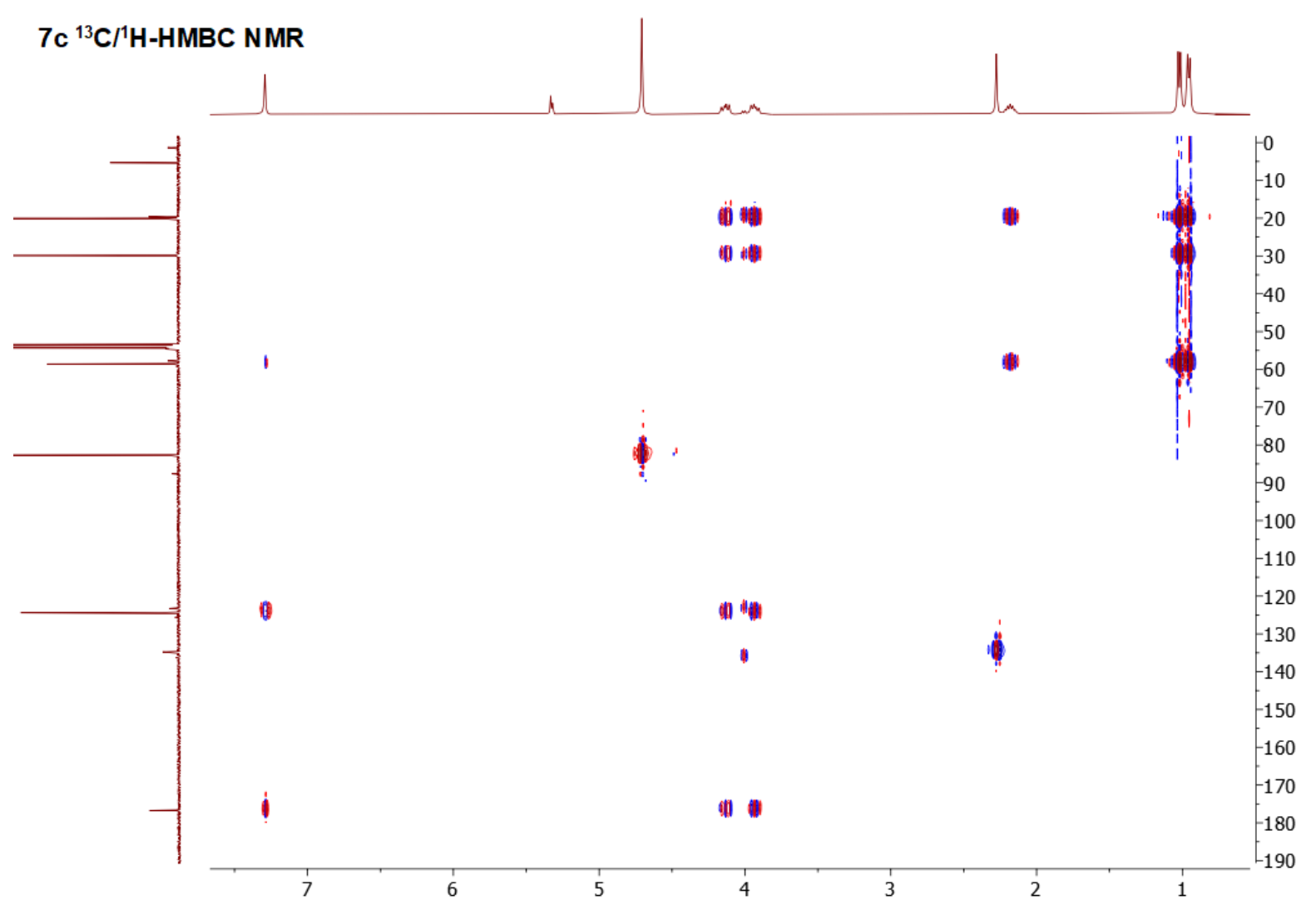

Figure S50. ${ }^{13} \mathrm{C} /{ }^{1} \mathrm{H}-\mathrm{HMBC}$ NMR spectrum $\left(\mathrm{CD}_{2} \mathrm{Cl}_{2}\right)$ for complex $7 \boldsymbol{c}$. 


\section{Structural analyses by NOE experiments}

Table S1. Approximated H...H distances based on crystal structures. $\mathrm{Cp}{ }^{*}$ complexes highlighted in grey

\begin{tabular}{|c|c|c|}
\hline Complex & Nearest protons & $\begin{array}{c}\text { Distance } \\
(\AA)\end{array}$ \\
\hline 3a & $\mathrm{Cp}-\mathrm{H} \ldots \mathrm{H}_{3} \mathrm{C}-\mathrm{N}$ & 2.564 \\
\hline 3b & $\mathrm{Cp}-\mathrm{H} \ldots \mathrm{HC}_{\mathrm{iPr}}$ & 2.249 \\
\hline $3 c$ & $\mathrm{Cp}-\mathrm{H} \ldots \mathrm{H}_{2} \mathrm{C}-\mathrm{N}$ & 2.738 \\
\hline 3f & $\mathrm{Cp}-\mathrm{H} \ldots \mathrm{H}_{3} \mathrm{C}-\mathrm{Mes}$ & 2.369 \\
\hline $3 \mathbf{e}$ & $\mathrm{Cp}-\mathrm{H} \ldots \mathrm{HC}_{\mathrm{iPr}}$ & 2.621 \\
\hline $4 a$ & $\mathrm{Cp}-\mathrm{CH}_{3} \ldots \mathrm{H}_{3} \mathrm{C}-\mathrm{N}$ & 2.180 \\
\hline $4 b$ & $\mathrm{Cp}-\mathrm{CH}_{3} \ldots \mathrm{HC}_{\mathrm{iPr}}$ & 2.118 \\
\hline $5 \mathbf{a}$ & $\mathrm{Cp}-\mathrm{H} \ldots \mathrm{H}_{3} \mathrm{C}-\mathrm{N}$ & 2.561 \\
\hline $5 \mathbf{b}$ & $\mathrm{Cp}-\mathrm{H} \ldots \mathrm{HC}_{\mathrm{iPr}}$ & 2.369 \\
\hline $5 c$ & $\mathrm{Cp}-\mathrm{H} \ldots \mathrm{H}_{2} \mathrm{C}-\mathrm{N}$ & 2.301 \\
\hline $5 e$ & $\mathrm{Cp}-\mathrm{H} \ldots \mathrm{HC}_{\mathrm{iPr}}$ & 2.523 \\
\hline $6 \mathbf{a}$ & $\mathrm{Cp}-\mathrm{CH}_{3} \ldots \mathrm{H}_{3} \mathrm{C}-\mathrm{N}$ & 2.354 \\
\hline $6 \mathbf{b}$ & $\mathrm{Cp}-\mathrm{CH}_{3} \ldots \mathrm{H}_{3} \mathrm{C}_{\mathrm{iPr}}$ & 2.232 \\
\hline
\end{tabular}

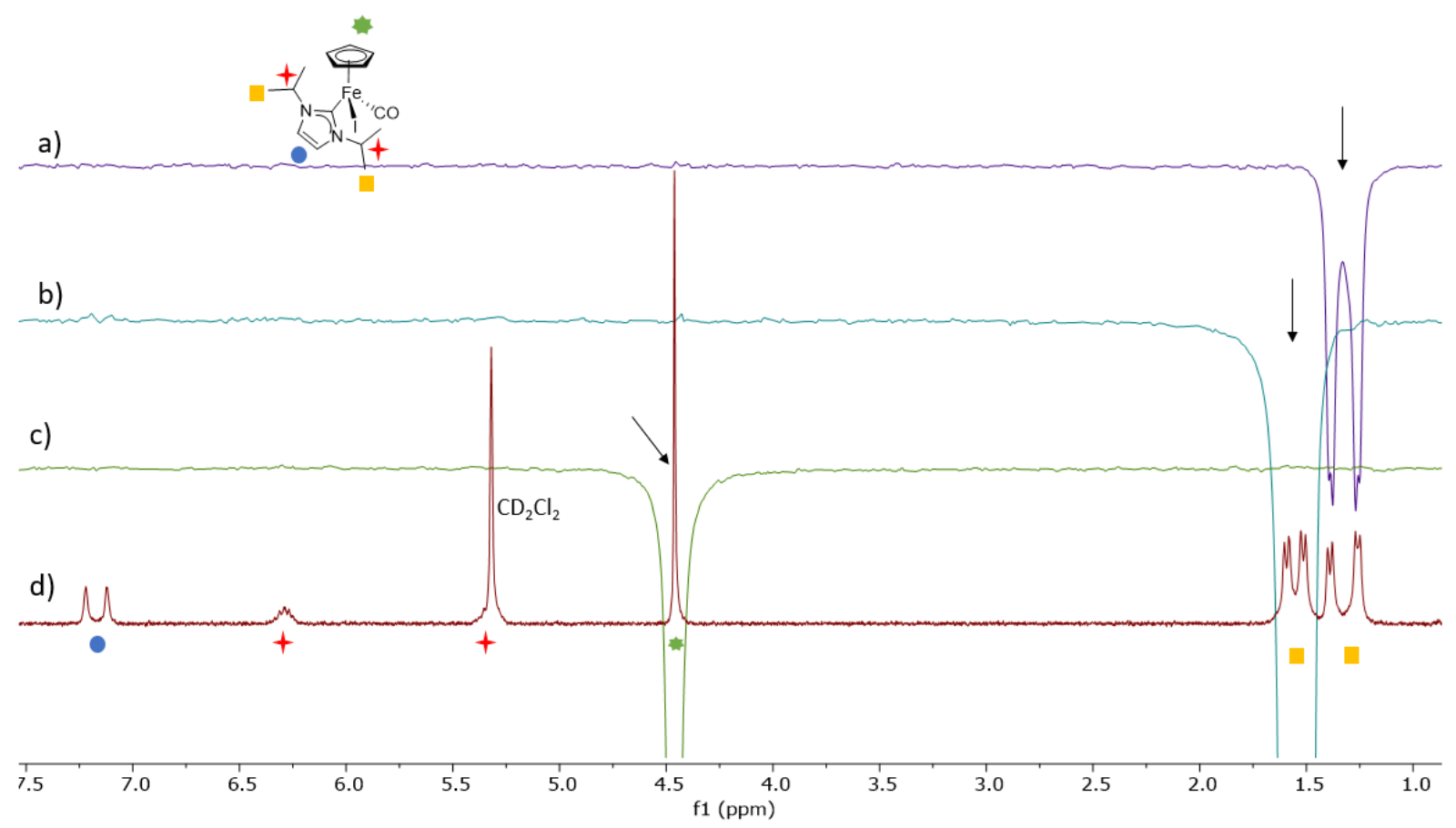

Figure S51. ${ }^{1} \mathrm{H} N O E$ spectra $\left(300 \mathrm{MHz}, \mathrm{CD}_{2} \mathrm{Cl}_{2}\right)$ for complex $\mathbf{5 b}$, revealing no NOE. Irradiated protons (inverse signals) are indicated with an arrow. a) and b) diastereotopic $\mathrm{CH}_{3}$ protons of the $\mathrm{iPr}$ group; c) Cp protons; d) normal ${ }^{l} H$ NMR spectrum. 


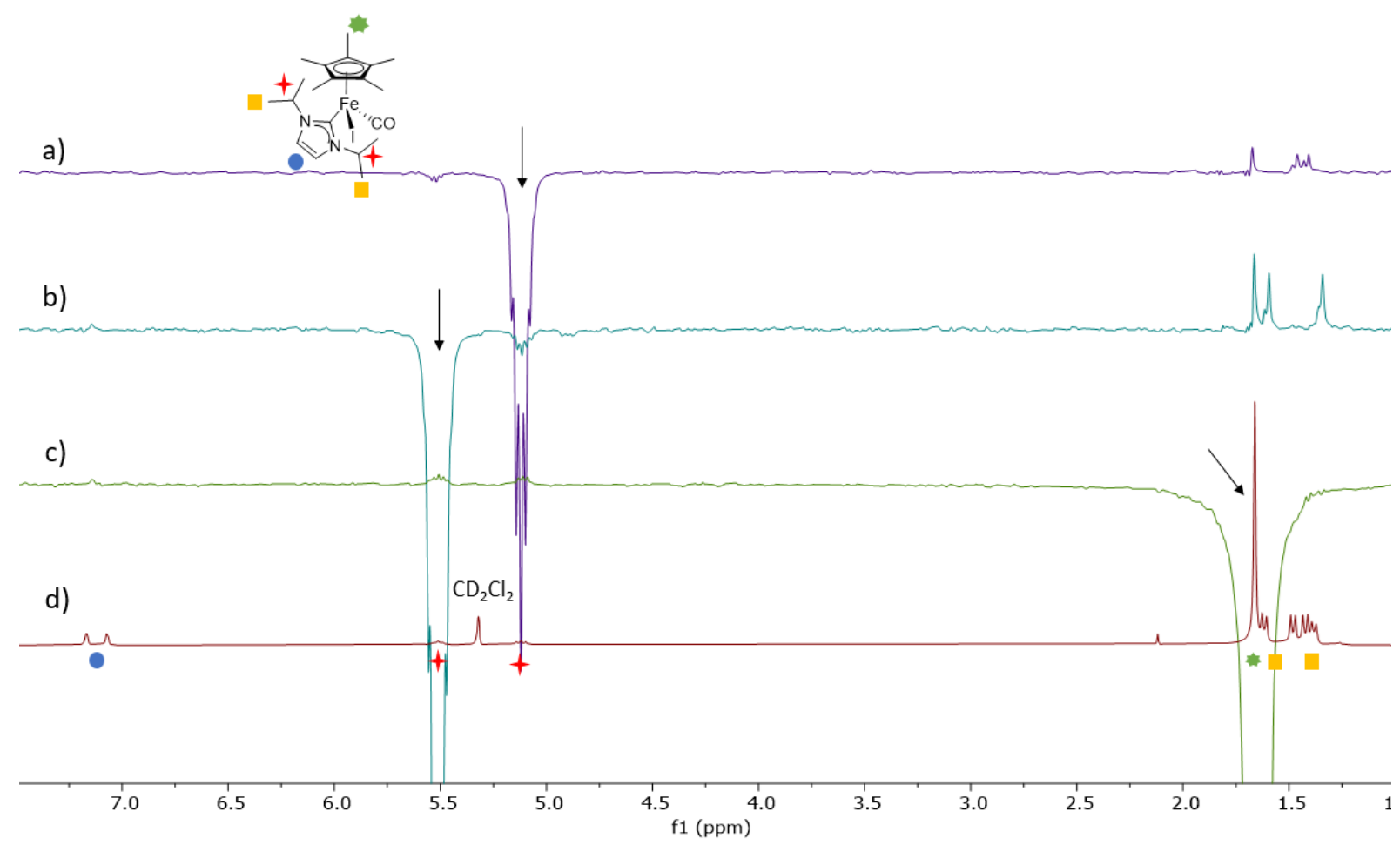

Figure S52. ${ }^{1} \mathrm{H} N O E$ spectra $\left(300 \mathrm{MHz}, \mathrm{CD}_{2} \mathrm{Cl}_{2}\right)$ for complex $\mathbf{6} \boldsymbol{b}$ revealing NOE between wingtip $\mathrm{CHMe}_{2}$ and $\mathrm{Cp}-\mathrm{CH}_{3}$ groups. Irradiated protons (inverse signals) are indicated with an arrow. a) and b) diastereotopic $\mathrm{CHMe}_{2}$ protons of the iPr group; c) $\mathrm{Cp}-\mathrm{CH}_{3}$ protons; d) normal ${ }^{1} \mathrm{H}$ NMR spectrum.

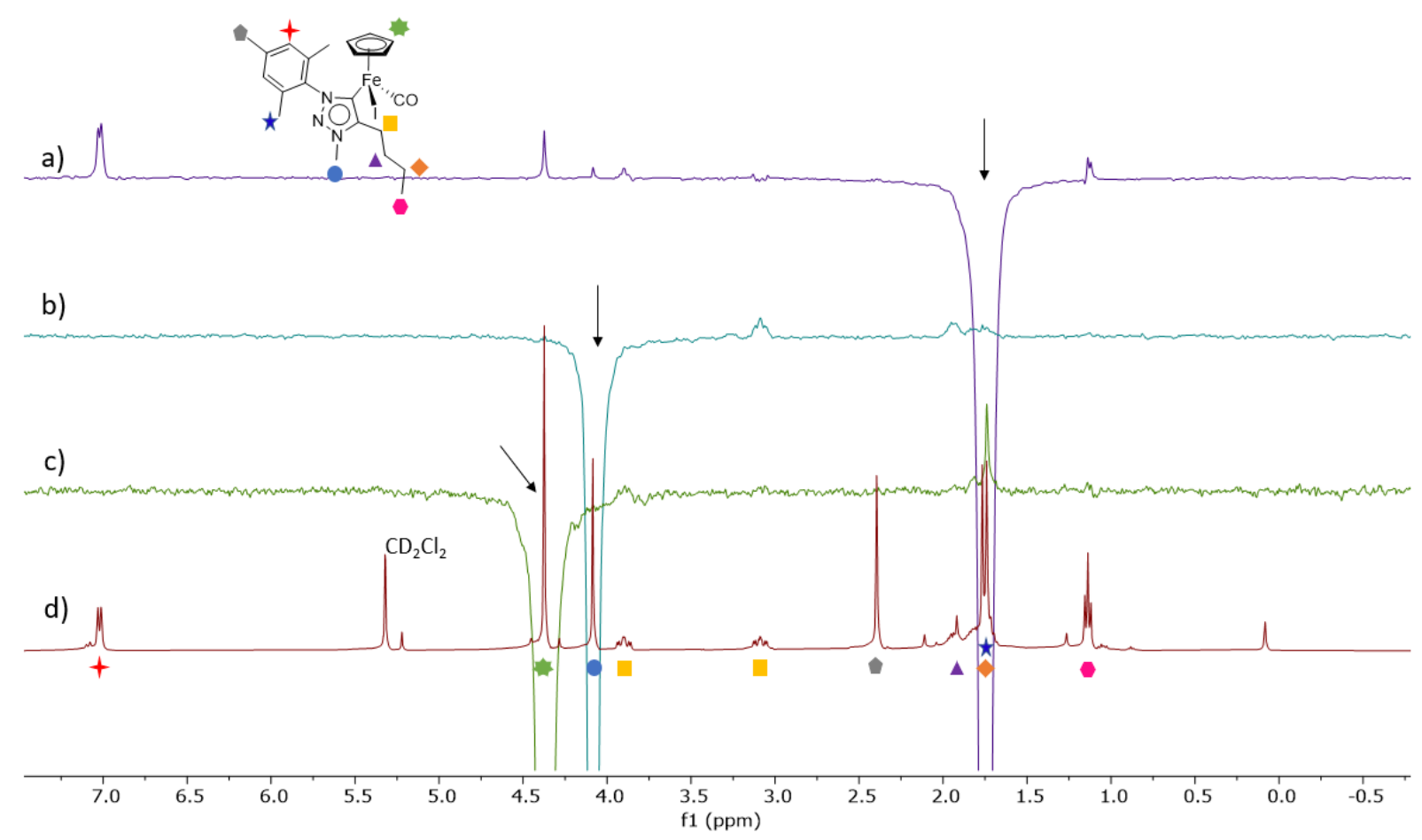

Figure S53. ${ }^{1} \mathrm{H} \mathrm{NOE}$ spectra $\left(300 \mathrm{MHz}, \mathrm{CD}_{2} \mathrm{Cl}_{2}\right)$ for complex $\mathbf{5 f}$ revealing interaction between the mesityl and Cp protons. Irradiated protons (inverse signals) are indicated with an arrow. a) mesityl ortho- $\mathrm{CH}_{3}$ group; b) $\mathrm{N}_{t r z}-\mathrm{CH}_{3}$ group; c) $\mathrm{Cp}-\mathrm{H}$ protons; d) normal ${ }^{1} \mathrm{H} \mathrm{NMR}$ spectrum. 


\section{Calculations for free energy of activation}

The free energy of activation was calculated according to the formula $\Delta G^{\sharp}=4.57 \mathrm{~T}_{\mathrm{c}}\left(9.97+\log \left(\mathrm{T}_{\mathrm{c}} / \delta v\right)\right.$ [cal mol ${ }^{-1}$ ] according to the values shown in Tables S2 and S3 and transformed in $\mathrm{kJ} \mathrm{mol}^{-1}$.

Table S2. Data used for calculating the free energy of activation $\Delta G^{t}$ for complex $\mathbf{5 c}$

\begin{tabular}{ccccc}
\hline & $\mathrm{C}_{\mathrm{imi}} \mathrm{H}$ & $\mathrm{CH}_{2}$ & $\mathrm{CH}$ & average \\
\hline $\mathrm{T}_{\mathrm{c}} / \mathrm{K}$ & 278.15 & 298.15 & 298.15 & \\
$\mathrm{~T}_{\mathrm{c}}-5 / \mathrm{K}$ & 273.15 & 293.15 & 293.15 & \\
$\mathrm{~T}_{\mathrm{c}}+5 / \mathrm{K}$ & 283.15 & 303.15 & 303.15 & \\
$\delta v$ & 56 & 225 & 93 & \\
\hline$\Delta G^{*} \mathrm{Tc}$ & 56.7 & 57.5 & 59.7 & 58.0 \\
$\Delta G_{\mathrm{Tc}-5}^{*}$ & 55.7 & 56.5 & 58.7 & 57.0 \\
$\Delta G^{*} \mathrm{Tc}+5$ & 57.8 & 58.5 & 60.8 & 59.0
\end{tabular}

The free energy of activation is given in $\mathrm{kJ} / \mathrm{mol}^{-1}(1 \mathrm{cal}=4.184 \mathrm{~J})$

Table S3. Data used for calculating the free energy of activation $\Delta G^{\ddagger}$ for complex $5 \boldsymbol{e}$

\begin{tabular}{cccc}
\hline & $\mathrm{C}_{\mathrm{imi}} \mathrm{H}$ & $\mathrm{CH}_{2}$ & average \\
\hline $\mathrm{T}_{\mathrm{c}} / \mathrm{K}$ & 328.15 & 318.15 & \\
$\mathrm{~T}_{\mathrm{c}}-5 / \mathrm{K}$ & 323.15 & 313.15 & \\
$\mathrm{~T}_{\mathrm{c}}+5 / \mathrm{K}$ & 333.15 & 323.15 & \\
$\delta v$ & 530 & 455 & 60.5 \\
\hline$\Delta G^{\ddagger} \mathrm{Tc}$ & 61.3 & 59.7 & 59.5 \\
$\Delta G^{\ddagger} \mathrm{Tc}-5$ & 60.3 & 58.7 & 61.5 \\
$\Delta G^{*} \mathrm{Tc}+5$ & 62.2 & 60.7 &
\end{tabular}

The free energy of activation is given in $\mathrm{kJ} / \mathrm{mol}^{-1}(1 \mathrm{cal}=4.184 \mathrm{~J})$ 


\section{Cyclic Voltammetry of neutral iron complexes}

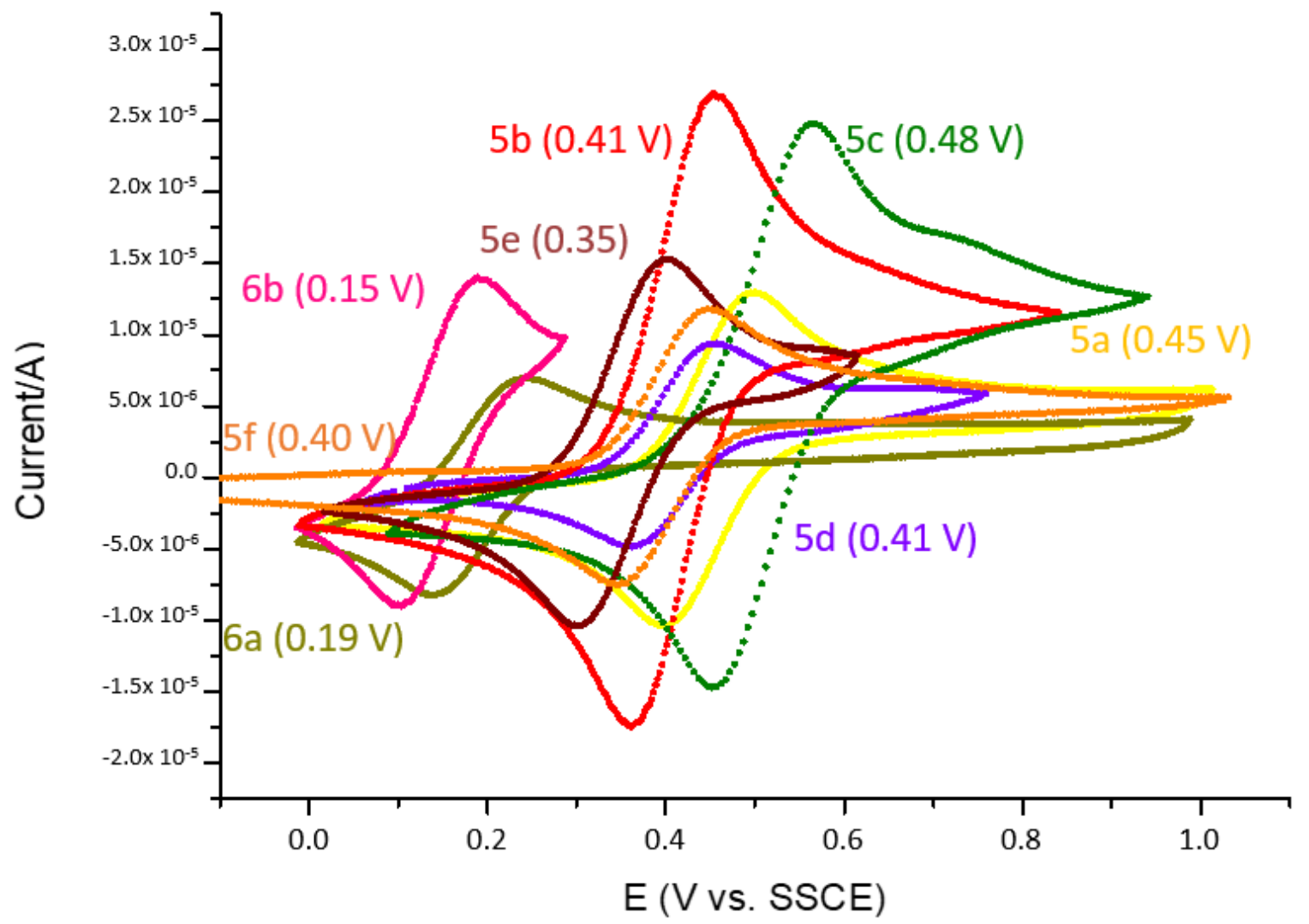

Figure S54. Cyclic voltammograms for iron complexes ( 1mM). Measured in $\mathrm{CH}_{2} \mathrm{Cl}_{2}, 0.1 \mathrm{M}$ $\left[B u_{4} N\right]\left[P F_{6}\right]$. In $V$ vs SSCE using $\left(E_{1 / 2}\left(F_{c}+/ F_{c}\right)\right.$ at $\left.+0.46 \mathrm{~V}\right)$ or decamethyl ferrocene $\left(E_{1 / 2}=+0.11 \mathrm{~V}\right.$, $\Delta E=0.10)$ as reference, scan rate $100 \mathrm{mV} \mathrm{s}^{-1}$. 


\section{Crystallographic details}

Table S4. Crystallographic data for cationic complexes $\mathbf{3 c}$ and $\mathbf{3 e}$

\begin{tabular}{|c|c|c|}
\hline & $3 \mathbf{c}$ & $3 \mathbf{e}$ \\
\hline CCDC No & 2072827 & 2072824 \\
\hline Colour, shape & Yellow plate & Yellow plate \\
\hline Empirical formula & $\mathrm{C}_{18} \mathrm{H}_{25} \mathrm{~F}_{1.06} \mathrm{FeI}_{0.82} \mathrm{~N}_{2} \mathrm{O}_{2} \mathrm{P}_{0.18}$ & $\mathrm{C}_{16} \mathrm{H}_{22} \mathrm{FeIN}_{3} \mathrm{O}_{2}$ \\
\hline Formula weight & 487.33 & 471.11 \\
\hline Temperature/K & $173.00(10)$ & $173.01(10)$ \\
\hline Crystal system & orthorhombic & triclinic \\
\hline Space group & $P b c n$ & P-1 \\
\hline $\mathrm{a} / \AA$ & $16.0541(2)$ & $9.5092(3)$ \\
\hline $\mathrm{b} / \AA ̊$ & $13.1849(3)$ & $10.1240(3)$ \\
\hline $\mathrm{c} / \AA$ & $19.5915(2)$ & $10.2881(3)$ \\
\hline$\alpha /^{\circ}$ & 90 & $87.331(3)$ \\
\hline$\beta /^{\circ}$ & 90 & $76.437(3)$ \\
\hline$\gamma /{ }^{\circ}$ & 90 & $77.552(3)$ \\
\hline Volume $/ \AA^{3}$ & $4146.96(11)$ & $940.19(6)$ \\
\hline Z & 8 & 2 \\
\hline$\rho_{\text {calc }} \mathrm{g} / \mathrm{cm}^{3}$ & 1.561 & 1.664 \\
\hline$\mu / \mathrm{mm}^{-1}$ & 1.987 & 2.455 \\
\hline $\mathrm{F}(000)$ & 1959.0 & 468.0 \\
\hline Crystal size $/ \mathrm{mm}^{3}$ & $0.307 \times 0.166 \times 0.046$ & $0.41 \times 0.194 \times 0.042$ \\
\hline Radiation & $\operatorname{MoK} \alpha(\lambda=0.71073)$ & $\operatorname{MoK} \alpha(\lambda=0.71073)$ \\
\hline Reflections collected & 53409 & 15969 \\
\hline \multirow{2}{*}{ Independent reflections } & $4921\left[\mathrm{R}_{\text {int }}=0.0380, \mathrm{R}_{\text {sigma }}=\right.$ & $4266\left[\mathrm{R}_{\text {int }}=0.0343, \mathrm{R}_{\text {sigma }}=\right.$ \\
\hline & $0.0189]$ & $0.0354]$ \\
\hline Data/restraints/parameters & $4921 / 222 / 263$ & $4266 / 0 / 213$ \\
\hline Goodness-of-fit on $\mathrm{F}^{2}$ & 1.372 & 1.039 \\
\hline Final $R$ indexes $[I>=2 \sigma(I)]$ & $\mathrm{R}_{1}=0.0686, \mathrm{wR}_{2}=0.1365$ & $\mathrm{R}_{1}=0.0293, \mathrm{wR}_{2}=0.0534$ \\
\hline Final $\mathrm{R}$ indexes [all data] & $\mathrm{R}_{1}=0.0756, \mathrm{wR}_{2}=0.1388$ & $\mathrm{R}_{1}=0.0412, \mathrm{wR}_{2}=0.0583$ \\
\hline $\begin{array}{l}\text { Largest diff. peak/hole / e } \\
\AA^{-3}\end{array}$ & $0.80 /-1.53$ & $0.60 /-0.45$ \\
\hline
\end{tabular}


Table S5. Crystallographic data for cationic complexes $4 \boldsymbol{a}$ and $\mathbf{4 b}$

\begin{tabular}{|c|c|c|}
\hline & $4 a$ & $4 b$ \\
\hline CCDC No & 2072829 & 2072831 \\
\hline Colour, shape & Yellow plate & Yellow plate \\
\hline Empirical formula & $\mathrm{C}_{18} \mathrm{H}_{25} \mathrm{Cl}_{2} \mathrm{FeIN}_{2} \mathrm{O}_{2}$ & $\mathrm{C}_{22} \mathrm{H}_{33} \mathrm{Cl}_{2} \mathrm{FeIN}_{2} \mathrm{O}_{2}$ \\
\hline Formula weight & 555.05 & 611.15 \\
\hline Temperature/K & $173.00(10)$ & $173.00(10)$ \\
\hline Crystal system & triclinic & triclinic \\
\hline Space group & $P-1$ & $P-1$ \\
\hline $\mathrm{a} / \AA$ & $8.4701(2)$ & $8.4573(4)$ \\
\hline $\mathrm{b} / \AA$ & $10.8999(3)$ & $11.3026(4)$ \\
\hline $\mathrm{c} / \AA$ & $12.8593(4)$ & $15.0799(7)$ \\
\hline$\alpha{ }^{\circ}$ & $100.916(3)$ & $82.696(3)$ \\
\hline$\beta /{ }^{\circ}$ & $102.450(2)$ & $77.784(4)$ \\
\hline$\gamma /{ }^{\circ}$ & $90.372(2)$ & $72.130(3)$ \\
\hline Volume $/ \AA^{3}$ & 1136.93(6) & $1337.87(11)$ \\
\hline Z & 2 & 2 \\
\hline$\rho_{\text {calc }} \mathrm{g} / \mathrm{cm}^{3}$ & 1.621 & 1.517 \\
\hline$\mu / \mathrm{mm}^{-1}$ & 2.270 & 1.936 \\
\hline $\mathrm{F}(000)$ & 552.0 & 616.0 \\
\hline Crystal size $/ \mathrm{mm}^{3}$ & $0.15 \times 0.1 \times 0.1$ & $0.2 \times 0.1 \times 0.1$ \\
\hline Radiation & $\operatorname{MoK} \alpha(\lambda=0.71073)$ & $\operatorname{MoK} \alpha(\lambda=0.71073)$ \\
\hline Reflections collected & 19462 & 18731 \\
\hline Independent reflections & $\begin{array}{l}5141\left[\mathrm{R}_{\text {int }}=0.0339, \mathrm{R}_{\text {sigma }}=\right. \\
0.0320]\end{array}$ & $18731\left[R_{\text {int }}=0.0755, R_{\text {sigma }}=0.0780\right]$ \\
\hline Data/restraints/parameters & $5141 / 0 / 242$ & $18731 / 0 / 301$ \\
\hline Goodness-of-fit on $\mathrm{F}^{2}$ & 1.062 & 1.012 \\
\hline Final $R$ indexes $[\mathrm{I}>=2 \sigma(\mathrm{I})]$ & $\mathrm{R}_{1}=0.0440, \mathrm{wR}_{2}=0.1076$ & $\mathrm{R}_{1}=0.0639, \mathrm{wR}_{2}=0.1737$ \\
\hline Final $\mathrm{R}$ indexes [all data] & $\mathrm{R}_{1}=0.0555, \mathrm{wR}_{2}=0.1157$ & $\mathrm{R}_{1}=0.1009, \mathrm{wR}_{2}=0.1862$ \\
\hline Largest diff. peak/hole / e $\AA^{-3}$ & $1.41 /-0.97$ & $2.29 /-0.64$ \\
\hline
\end{tabular}


Table S6. Crystallographic data for neutral complexes $\mathbf{5 c}$ and $\mathbf{5 e}$

\begin{tabular}{|c|c|c|}
\hline & $5 c$ & $5 e$ \\
\hline CCDC No & 2072826 & 2072828 \\
\hline Colour, shape & Green, plate & Green, plate \\
\hline Empirical formula & $\mathrm{C}_{17} \mathrm{H}_{25} \mathrm{FeIN}{ }_{2} \mathrm{O}$ & $\mathrm{C}_{15} \mathrm{H}_{22} \mathrm{FeIN}{ }_{3} \mathrm{O}$ \\
\hline Formula weight & 456.14 & 443.10 \\
\hline Temperature/K & $173.00(10)$ & $173.00(10)$ \\
\hline Crystal system & triclinic & monoclinic \\
\hline Space group & $\mathrm{P}-1$ & $P 2_{1} / c$ \\
\hline $\mathrm{a} / \AA$ & $8.5808(3)$ & $13.6072(8)$ \\
\hline $\mathrm{b} / \AA$ & $9.1020(3)$ & $9.4684(4)$ \\
\hline $\mathrm{c} / \AA$ & $12.3043(4)$ & $14.2383(11)$ \\
\hline$\alpha /^{\circ}$ & $72.940(3)$ & 90 \\
\hline$\beta /{ }^{\circ}$ & $82.272(3)$ & $101.312(7)$ \\
\hline$\gamma /{ }^{\circ}$ & $89.979(2)$ & 90 \\
\hline Volume $/ \AA^{3}$ & $909.60(5)$ & $1798.8(2)$ \\
\hline Z & 2 & 4 \\
\hline$\rho_{\text {calc }} \mathrm{g} / \mathrm{cm}^{3}$ & 1.665 & 1.636 \\
\hline$\mu / \mathrm{mm}^{-1}$ & 2.529 & 2.557 \\
\hline $\mathrm{F}(000)$ & 456.0 & 880.0 \\
\hline Crystal size $/ \mathrm{mm}^{3}$ & $0.579 \times 0.327 \times 0.186$ & $0.12 \times 0.05 \times 0.025$ \\
\hline Radiation & $\operatorname{MoK} \alpha(\lambda=0.71073)$ & $\operatorname{MoK} \alpha(\lambda=0.71073)$ \\
\hline Reflections collected & 19214 & 15052 \\
\hline \multirow{2}{*}{ Independent reflections } & $4140\left[\mathrm{R}_{\text {int }}=0.0345, \mathrm{R}_{\text {sigma }}=\right.$ & $3952\left[\mathrm{R}_{\text {int }}=0.0352, \mathrm{R}_{\text {sigma }}=\right.$ \\
\hline & $0.0260]$ & $0.0336]$ \\
\hline Data/restraints/parameters & $4140 / 0 / 203$ & $3952 / 0 / 195$ \\
\hline Goodness-of-fit on $\mathrm{F}^{2}$ & 1.058 & 1.046 \\
\hline Final $R$ indexes $[\mathrm{I}>=2 \sigma(\mathrm{I})]$ & $\mathrm{R}_{1}=0.0203, \mathrm{wR}_{2}=0.0467$ & $\mathrm{R}_{1}=0.0375, \mathrm{wR}_{2}=0.0740$ \\
\hline Final $\mathrm{R}$ indexes [all data] & $\mathrm{R}_{1}=0.0236, \mathrm{wR}_{2}=0.0483$ & $\mathrm{R}_{1}=0.0573, \mathrm{wR}_{2}=0.0842$ \\
\hline Largest diff. peak/hole / e $\AA^{-3}$ & $0.44 /-0.60$ & $1.05 /-1.31$ \\
\hline
\end{tabular}


Table S7. Crystallographic data for neutral complexes $\mathbf{6} \boldsymbol{a}$ and $\mathbf{6 b}$

\begin{tabular}{|c|c|c|}
\hline & $6 a$ & $6 \mathbf{b}$ \\
\hline CCDC No & 2072825 & 2072832 \\
\hline Colour, shape & Green, plate & Green, plate \\
\hline Empirical formula & $\mathrm{C}_{16} \mathrm{H}_{23} \mathrm{FeIN}{ }_{2} \mathrm{O}$ & $\mathrm{C}_{20} \mathrm{H}_{31} \mathrm{FeIN} \mathrm{N}_{2} \mathrm{O}$ \\
\hline Formula weight & 442.11 & 498.22 \\
\hline Temperature/K & $123.01(10)$ & $173.00(10)$ \\
\hline Crystal system & monoclinic & orthorhombic \\
\hline Space group & $\mathrm{P} 2{ }_{1} / \mathrm{n}$ & $\mathrm{Pbca}$ \\
\hline $\mathrm{a} / \AA$ & $9.39940(17)$ & $14.75800(10)$ \\
\hline $\mathrm{b} / \AA$ & $12.53647(16)$ & $16.7791(2)$ \\
\hline $\mathrm{c} / \AA$ & $14.3887(3)$ & $17.6495(2)$ \\
\hline$\alpha /^{\circ}$ & 90 & 90 \\
\hline$\beta /{ }^{\circ}$ & $95.3883(18)$ & 90 \\
\hline$\gamma /{ }^{\circ}$ & 90 & 90 \\
\hline Volume $/ \AA^{3}$ & $1688.00(5)$ & $4370.48(8)$ \\
\hline Z & 4 & 8 \\
\hline$\rho_{\text {calc }} \mathrm{g} / \mathrm{cm}^{3}$ & 1.740 & 1.514 \\
\hline$\mu / \mathrm{mm}^{-1}$ & 2.723 & 2.113 \\
\hline $\mathrm{F}(000)$ & 880.0 & 2016.0 \\
\hline Crystal size $/ \mathrm{mm}^{3}$ & $0.25 \times 0.15 \times 0.15$ & $0.25 \times 0.25 \times 0.05$ \\
\hline Radiation & $\operatorname{MoK} \alpha(\lambda=0.71073)$ & $\operatorname{MoK} \alpha(\lambda=0.71073)$ \\
\hline Reflections collected & 14083 & 19448 \\
\hline \multirow{2}{*}{ Independent reflections } & $3700\left[\mathrm{R}_{\text {int }}=0.0427, \mathrm{R}_{\text {sigma }}=\right.$ & $4927\left[\mathrm{R}_{\text {int }}=0.0299, \mathrm{R}_{\text {sigma }}=\right.$ \\
\hline & $0.0431]$ & $0.0272]$ \\
\hline Data/restraints/parameters & $3700 / 0 / 197$ & $4927 / 1 / 235$ \\
\hline Goodness-of-fit on $\mathrm{F}^{2}$ & 1.087 & 1.058 \\
\hline Final R indexes $[\mathrm{I}>=2 \sigma(\mathrm{I})]$ & $\mathrm{R}_{1}=0.0293, \mathrm{wR}_{2}=0.0621$ & $\mathrm{R}_{1}=0.0467, \mathrm{wR}_{2}=0.1406$ \\
\hline Final $\mathrm{R}$ indexes [all data] & $\mathrm{R}_{1}=0.0362, \mathrm{wR}_{2}=0.0660$ & $\mathrm{R}_{1}=0.0543, \mathrm{wR}_{2}=0.1482$ \\
\hline Largest diff. peak/hole / e $\AA^{-3}$ & $0.84 /-0.66$ & $1.86 /-1.78$ \\
\hline
\end{tabular}


Table S8. Crystallographic data for cationic complex $7 c$

\begin{tabular}{|c|c|}
\hline CCDC No & 2072830 \\
\hline Colour, shape & Red, plate \\
\hline Empirical formula & $\mathrm{C}_{19} \mathrm{H}_{28} \mathrm{~F}_{6} \mathrm{FeN}_{3} \mathrm{OP}$ \\
\hline Formula weight & 515.26 \\
\hline Temperature/K & $173.00(10)$ \\
\hline Crystal system & monoclinic \\
\hline Space group & $\mathrm{P} 2{ }_{1} / \mathrm{c}$ \\
\hline $\mathrm{a} / \AA$ & $10.79716(10)$ \\
\hline $\mathrm{b} / \AA$ & $13.10798(12)$ \\
\hline $\mathrm{c} / \AA$ & $16.03200(14)$ \\
\hline$\alpha /^{\circ}$ & 90 \\
\hline$\beta /{ }^{\circ}$ & $95.0269(8)$ \\
\hline$\gamma /{ }^{\circ}$ & 90 \\
\hline Volume $/ \AA^{3}$ & $2260.26(3)$ \\
\hline Z & 4 \\
\hline$\rho_{\text {calc }} \mathrm{g} / \mathrm{cm}^{3}$ & 1.514 \\
\hline$\mu / \mathrm{mm}^{-1}$ & 0.803 \\
\hline $\mathrm{F}(000)$ & 1064.0 \\
\hline Crystal size $/ \mathrm{mm}^{3}$ & $0.334 \times 0.318 \times 0.069$ \\
\hline Radiation & $\operatorname{MoK} \alpha(\lambda=0.71073)$ \\
\hline Reflections collected & 24677 \\
\hline \multirow{2}{*}{ Independent reflections } & $5165\left[\mathrm{R}_{\text {int }}=0.0315, \mathrm{R}_{\text {sigma }}=\right.$ \\
\hline & $0.0252]$ \\
\hline Data/restraints/parameters & $5165 / 0 / 285$ \\
\hline Goodness-of-fit on $\mathrm{F}^{2}$ & 1.030 \\
\hline Final $R$ indexes $[\mathrm{I}>=2 \sigma(\mathrm{I})]$ & $\mathrm{R}_{1}=0.0320, \mathrm{wR}_{2}=0.0747$ \\
\hline Final $\mathrm{R}$ indexes [all data] & $\mathrm{R}_{1}=0.0395, \mathrm{wR}_{2}=0.0793$ \\
\hline Largest diff. peak/hole / e $\AA^{-3}$ & $0.35 /-0.29$ \\
\hline
\end{tabular}




\section{Steric maps of neutral NHC iron complexes}

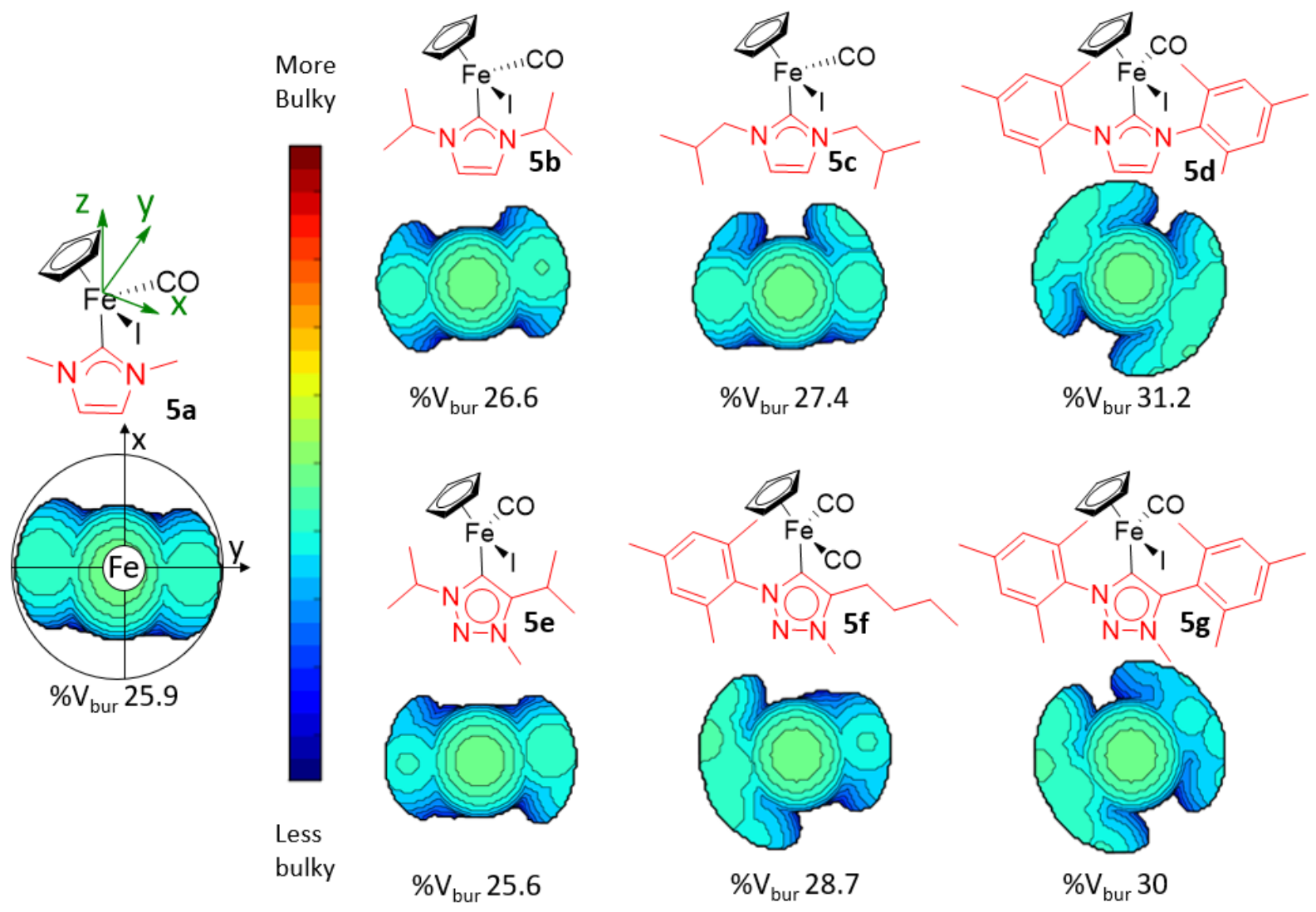

Figure S55. Steric maps of NHC iron complexes. Steric contribution have been calculated by using the SambVca software. ${ }^{\mathrm{S}-\mathrm{S} 11}$ The orientation of the ligand for $\mathbf{5 a}$ applies to all systems in this figure. For comparison between $C p$ and $C p^{*}$ complexes (not in figure): $\mathbf{6 a} \% V_{\text {bur }} 25.8, \boldsymbol{6} \boldsymbol{b} \% V_{\text {bur }} 26.2$. 


\section{Catalytic details}
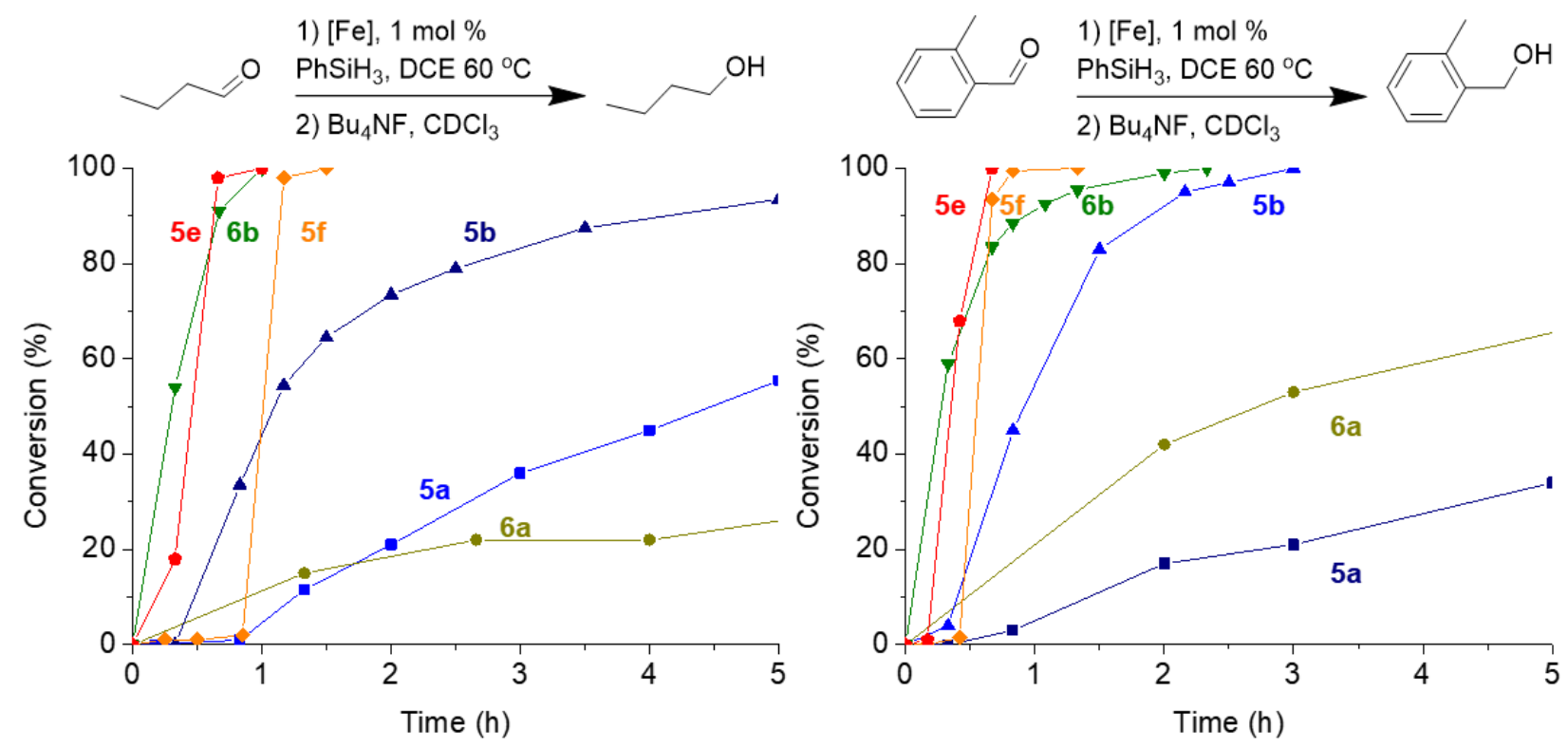

Figure S56. Time-conversion profiles for the hydrosilylation of butyraldehyde (left) and o-tolualdehyde (right) in 1,2-dichloroethane catalysed by iron(II) complexes.
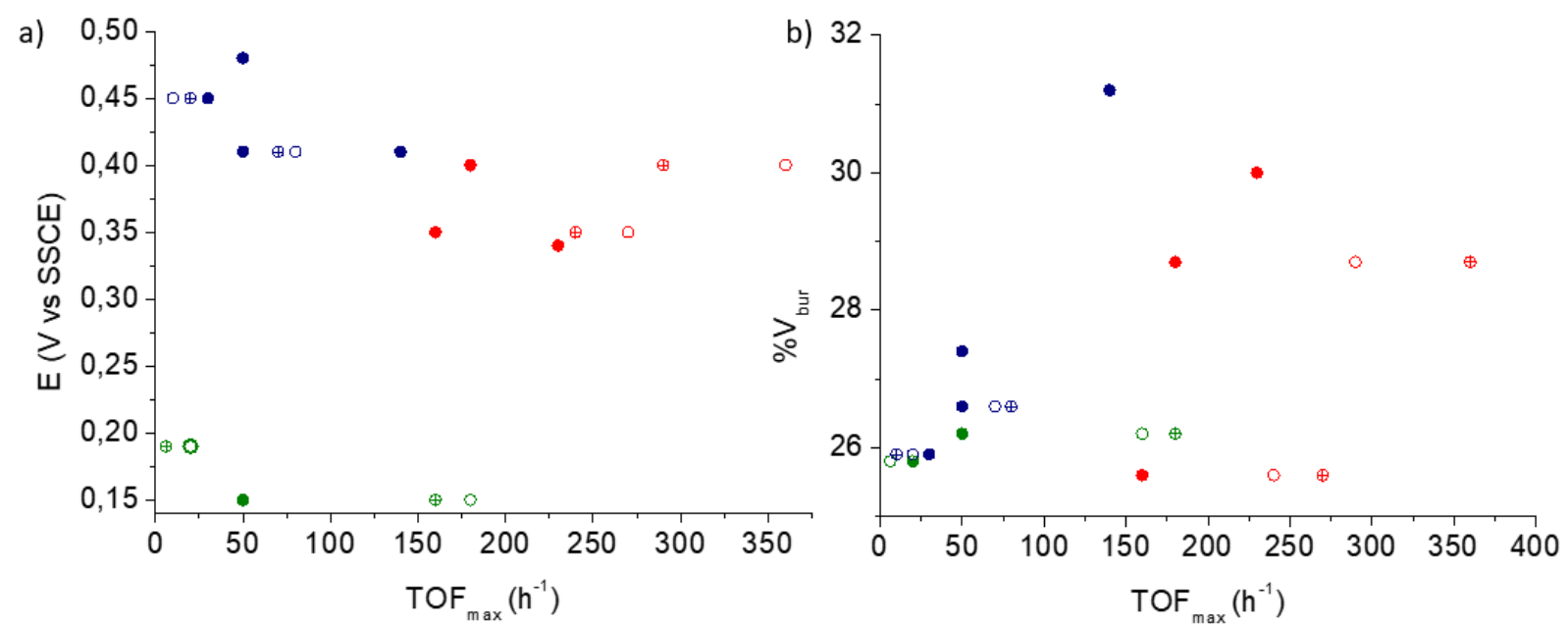

Figure S57. left: Plot of oxidation potentials $\left(E_{1 / 2}\right)$ vs TOF $F_{\max }$ for the catalytic conversion of 4nitrobenzaldehyde (full circles $\bullet$ ), for tolualdehyde (open circles, ○), and for butyraldehyde (semi-filled circles, $\oplus)$ and b) buried volume $\left(\% V_{b u r}\right)$ vs TOF $_{\max }$ for iron complexes with imi/Cp (blue), $\mathrm{trz} / \mathrm{Cp}$ (red) and $\mathrm{imi} / \mathrm{Cp}$ (green) ligand sets. 


\section{Mechanistic experiments}

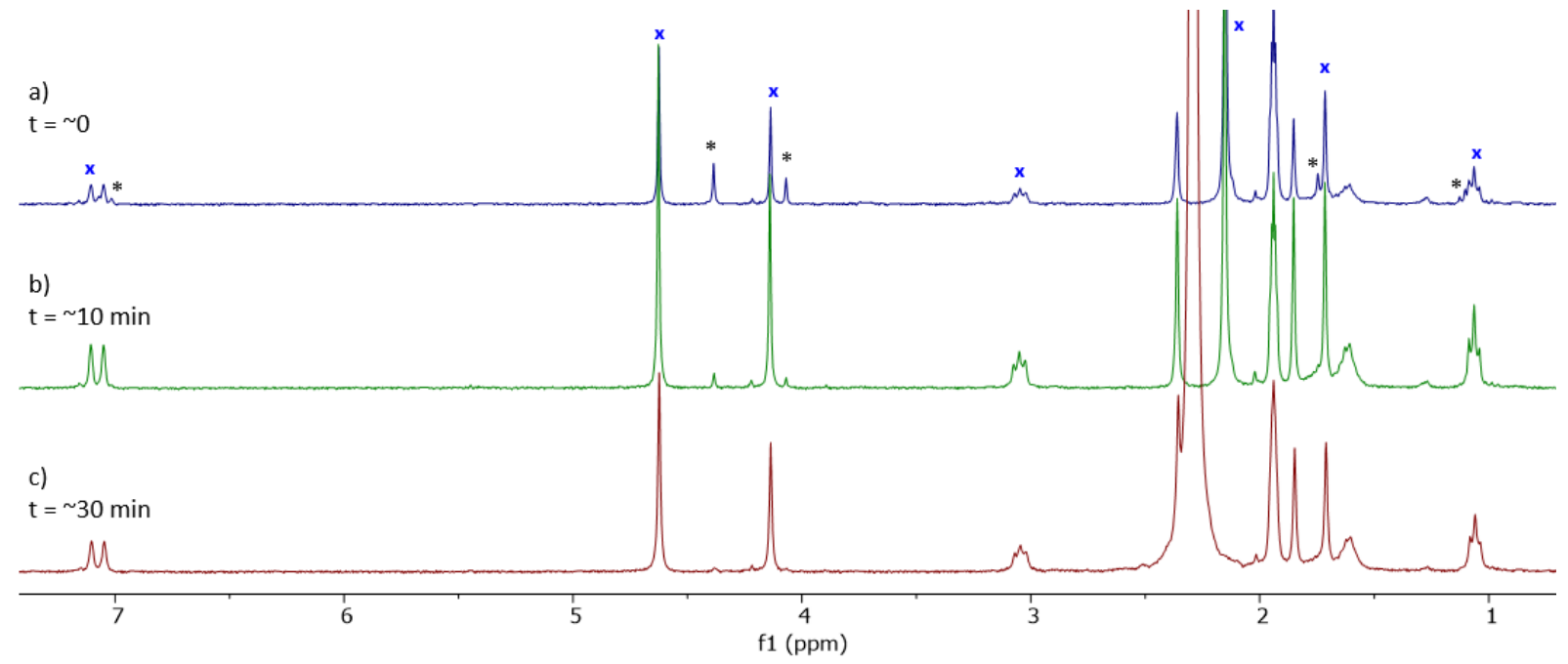

Figure S58. ${ }^{1} \mathrm{H} N M R$ spectra (300 MHz) of complex $\mathbf{5 f}$ in $\mathrm{CD}_{3} \mathrm{CN}$ and the formation of the proposed corresponding solvento complex, a) after addition of complex in $\mathrm{CD}_{3} \mathrm{CN}$; b) after $10 \mathrm{~min}$; c) after 30 $\min$.

Table S9. Hydrosilylation of 4-nitrobenzaldehyde by iron(II) complexes $\mathbf{5 c}$ and $7 \boldsymbol{c}^{a}$<smiles>O=Cc1ccc([N+](=O)[O-])cc1</smiles>

\begin{tabular}{ccccc}
\hline entry & complex & Additive & time (h) & conversion (\%) $^{\mathrm{b}}$ \\
\hline 1 & $5 \mathrm{c}$ & - & 3 & 100 \\
2 & $7 \mathrm{c}$ & - & $20 / 70$ & $30 / 68$ \\
3 & $7 \mathrm{c}$ & $\mathrm{LiOPr}^{(1.1 \mathrm{eq})}$ & 20 & 10 \\
4 & $7 \mathrm{c}$ & $\mathrm{NABH}_{4}($ excess $)$ & $20 / 70$ & $15 / 28$ \\
5 & $7 \mathrm{c}$ & $\mathrm{I}_{2}(0.6 \mathrm{eq})$ & $20 / 70$ & $16 / 37$ \\
\hline
\end{tabular}

${ }^{a}$ General conditions: substrate $(0.5 \mathrm{mmol}), \mathrm{PhSiH}_{3}(0.6 \mathrm{mmol}),[\mathrm{Fe}]$ complex $(5 \mu \mathrm{mol}, 1 \mathrm{~mol} \%), \mathrm{C}_{6} \mathrm{Me}_{6}$ as internal standard $(50 \mu \mathrm{mol}), 1,2$-dichloroethane $(2.5 \mathrm{~mL}), 60{ }^{\circ} \mathrm{C} .{ }^{b}$ Conversion determined by ${ }^{l} \mathrm{H}$ NMR spectroscopy. 

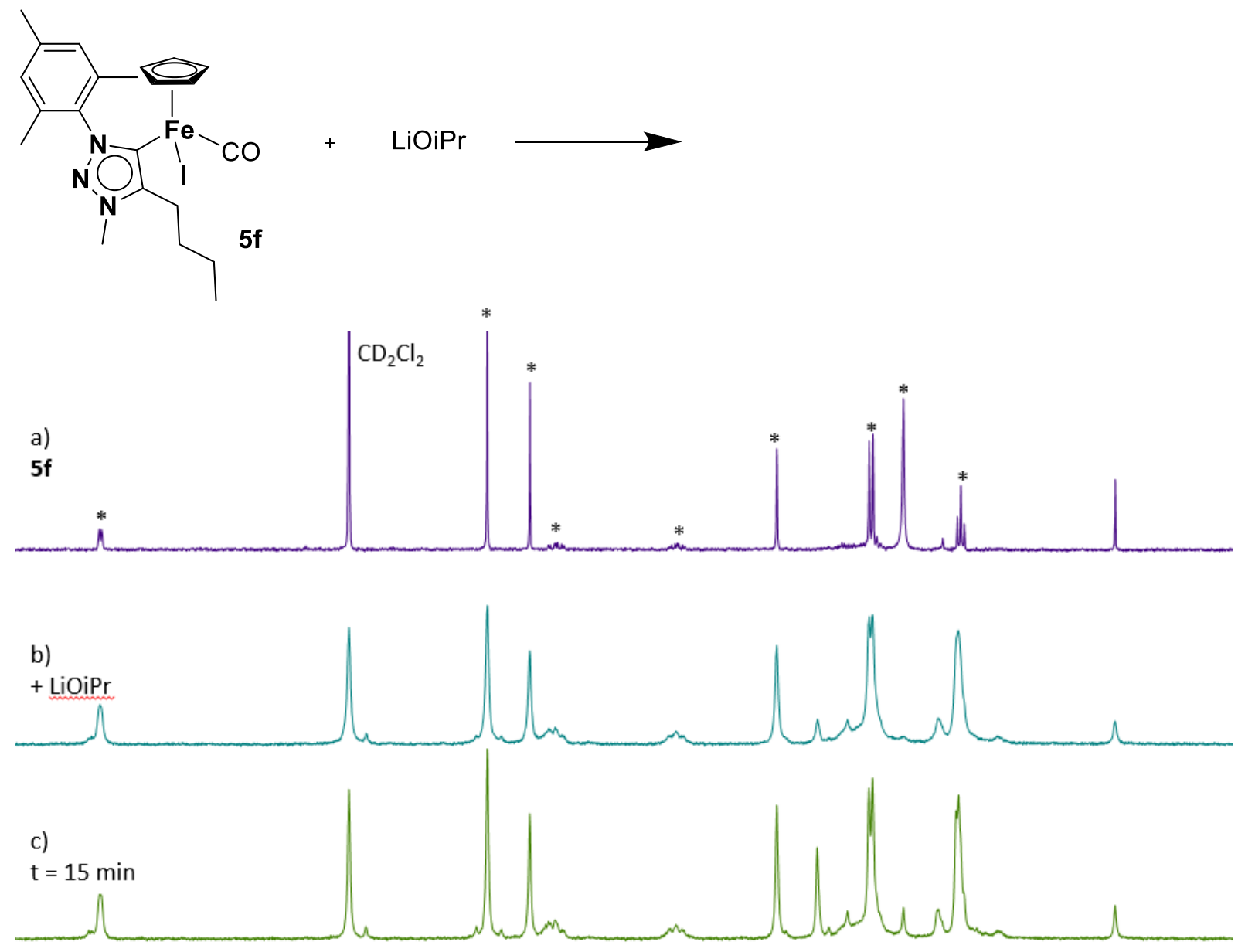

d)

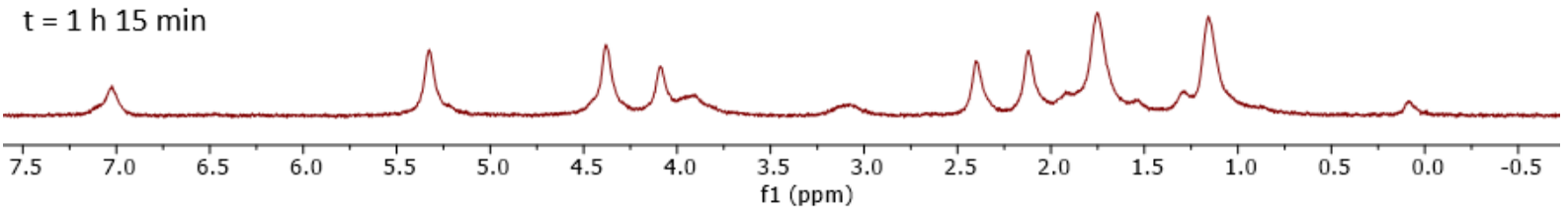

Figure S59. ${ }^{1} \mathrm{H} N \mathrm{NR}$ spectra (300 MHz) of a) complex $\mathbf{5 f}$ in $\mathrm{CD}_{2} \mathrm{Cl}_{2}$. b) complex $\mathbf{5 f}(2.7 \mathrm{mg}, 5$ umol, *) and LiOiPr $(5.5 \mu \mathrm{mol})$ in $\mathrm{CD}_{2} \mathrm{Cl}_{2}(0.5 \mathrm{~mL})$. c) after $15 \mathrm{~min}$ at $60^{\circ} \mathrm{C}$, and d) after $1 \mathrm{~h} 15 \mathrm{~min}$ at 60 ${ }^{\circ} \mathrm{C}$. 

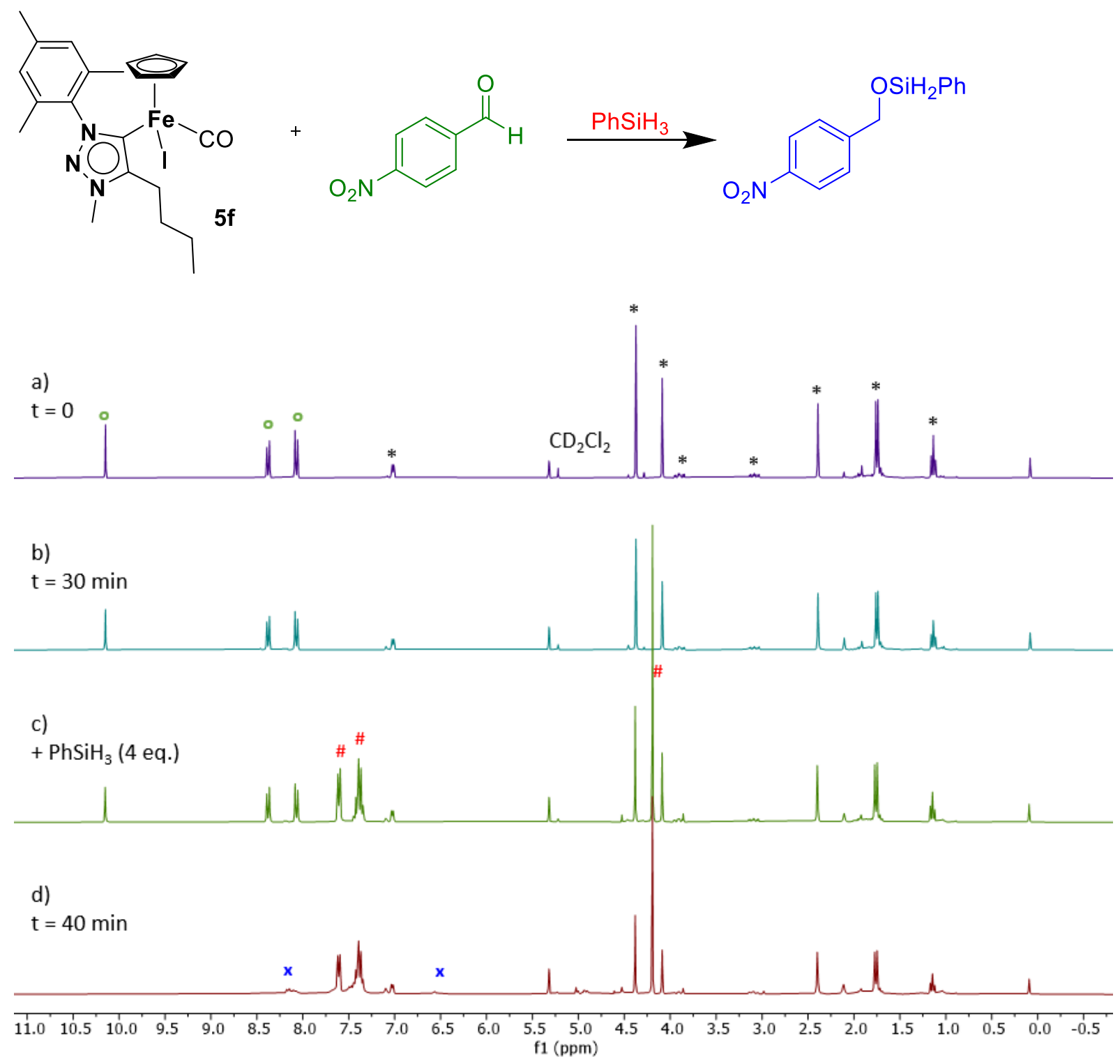

Figure S60. ${ }^{1} \mathrm{H}$ NMR spectra $(300 \mathrm{MHz})$ of complex $\mathbf{5 f}(8 \mathrm{mg}, 15 \mu \mathrm{mol}$, indicated with *) and 4nitrobenzaldehyde (30 $\mu \mathrm{mol}$, indicated with o) in $\mathrm{CD}_{2} \mathrm{Cl}_{2}(0.5 \mathrm{~mL})$, a) start, b) 30 min at $60^{\circ} \mathrm{C}$, c) added $\mathrm{PhSiH}_{3}$ (4 eq., indicated with \#), d) heated mixture c at $60{ }^{\circ} \mathrm{C}$ for additional 10 min, new signals indicated with $\mathbf{x}$. 


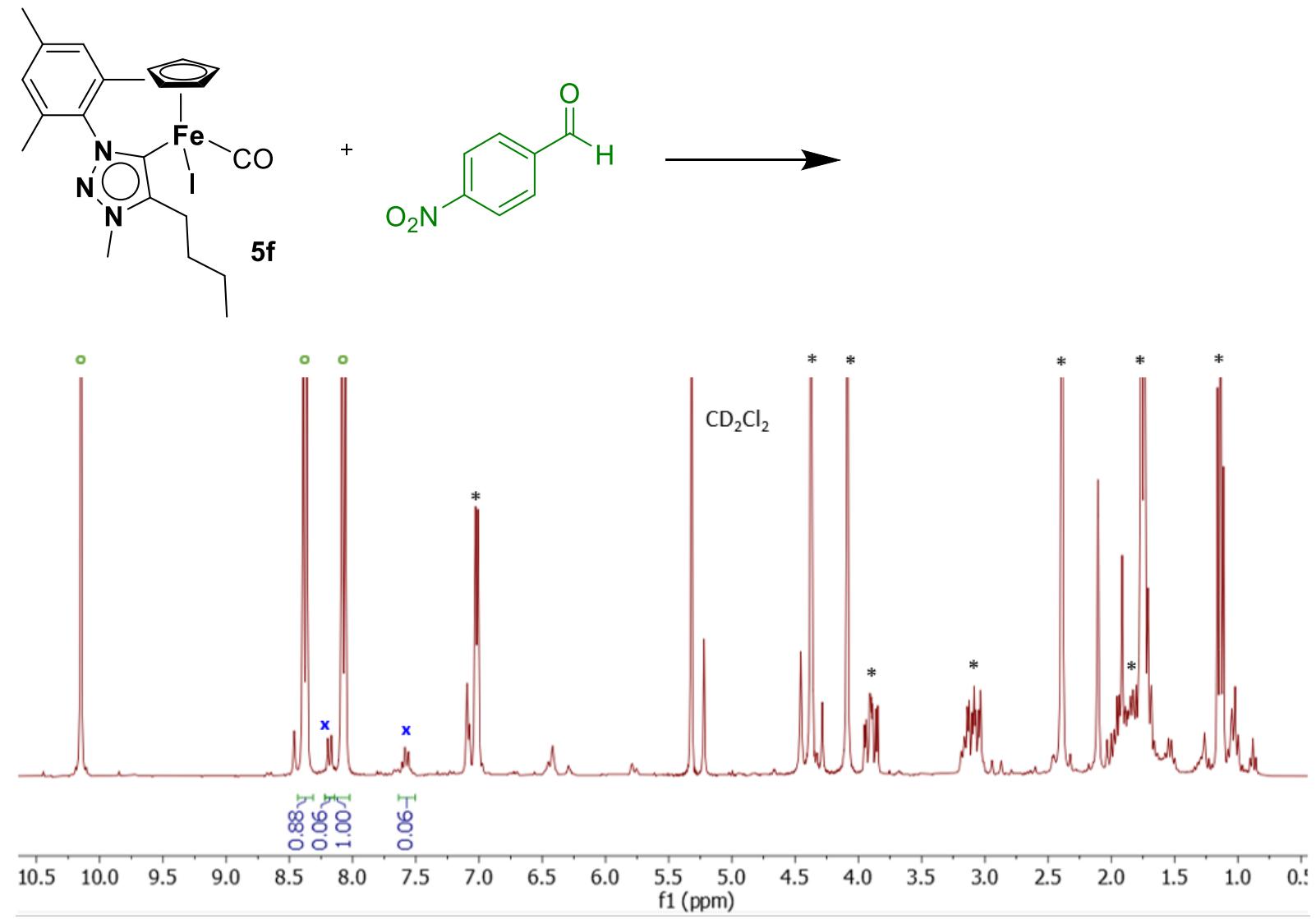

Figure S61. ${ }^{1} \mathrm{H}$ NMR spectra (300 MHz) of complex $\mathbf{5 f}(8 \mathrm{mg}, 15 \mu \mathrm{mol}$, indicated with *) and 4nitrobenzaldehyde (30 $\mu \mathrm{mol}$, indicated with o) in $\mathrm{CD}_{2} \mathrm{Cl}_{2}(0.5 \mathrm{~mL})$, heated for $30 \mathrm{~min}$, new signals indicated with $\mathrm{x}$. 


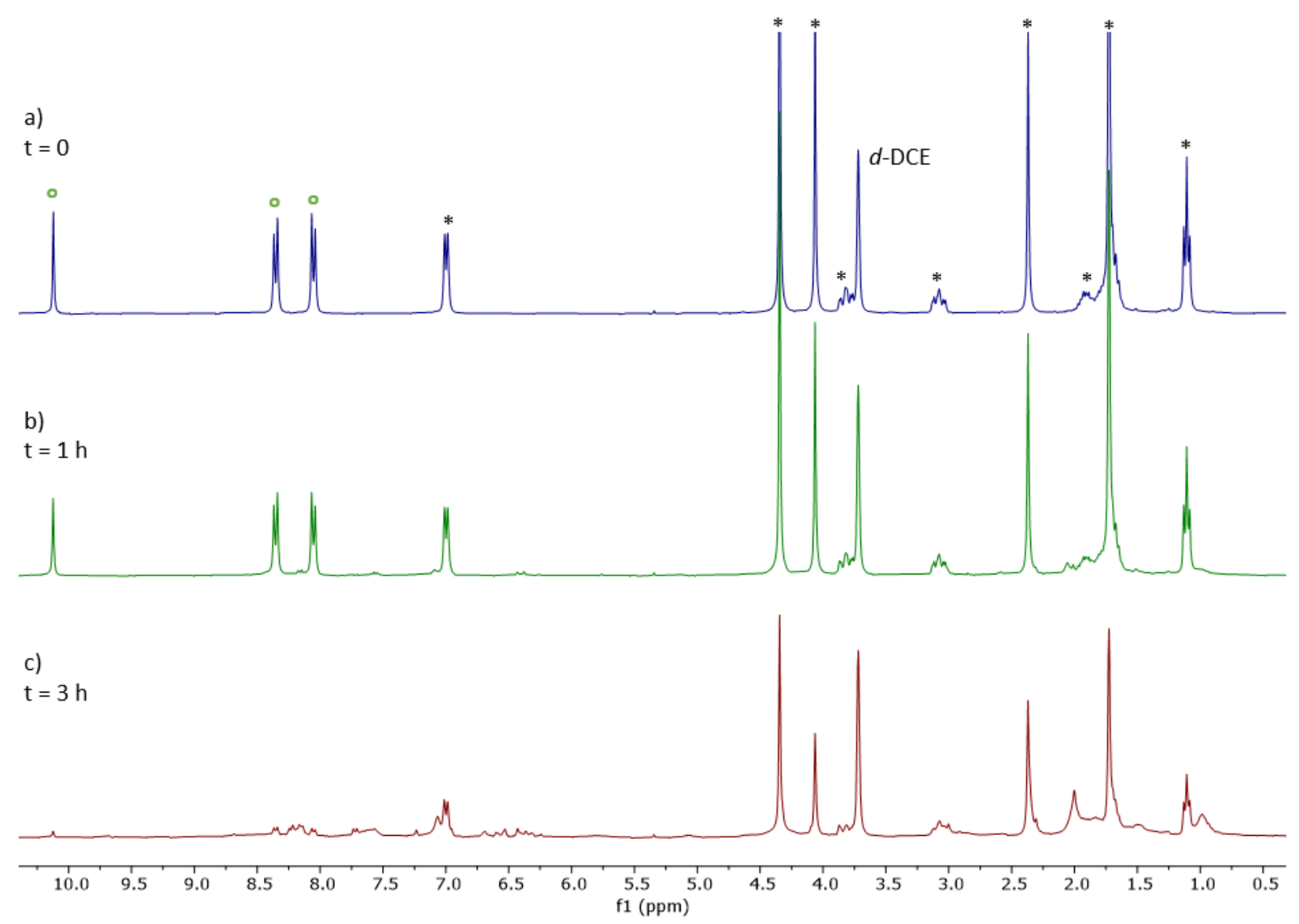

Figure S62. ${ }^{1} \mathrm{H}$ NMR spectra (300 MHz) of complex $\mathbf{5 f}(8 \mathrm{mg}, 15 \mu \mathrm{mol}$, indicated with *) and 4nitrobenzaldehyde $(2.4 \mathrm{mg}, 15 \mu \mathrm{mol}$, indicated with 0$)$ in 1,2-dichloroethane- $\mathrm{d}_{4}(0.5 \mathrm{~mL}), 0 \mathrm{~h}, 1 \mathrm{~h}$ and $3 \mathrm{~h}$ from the top.

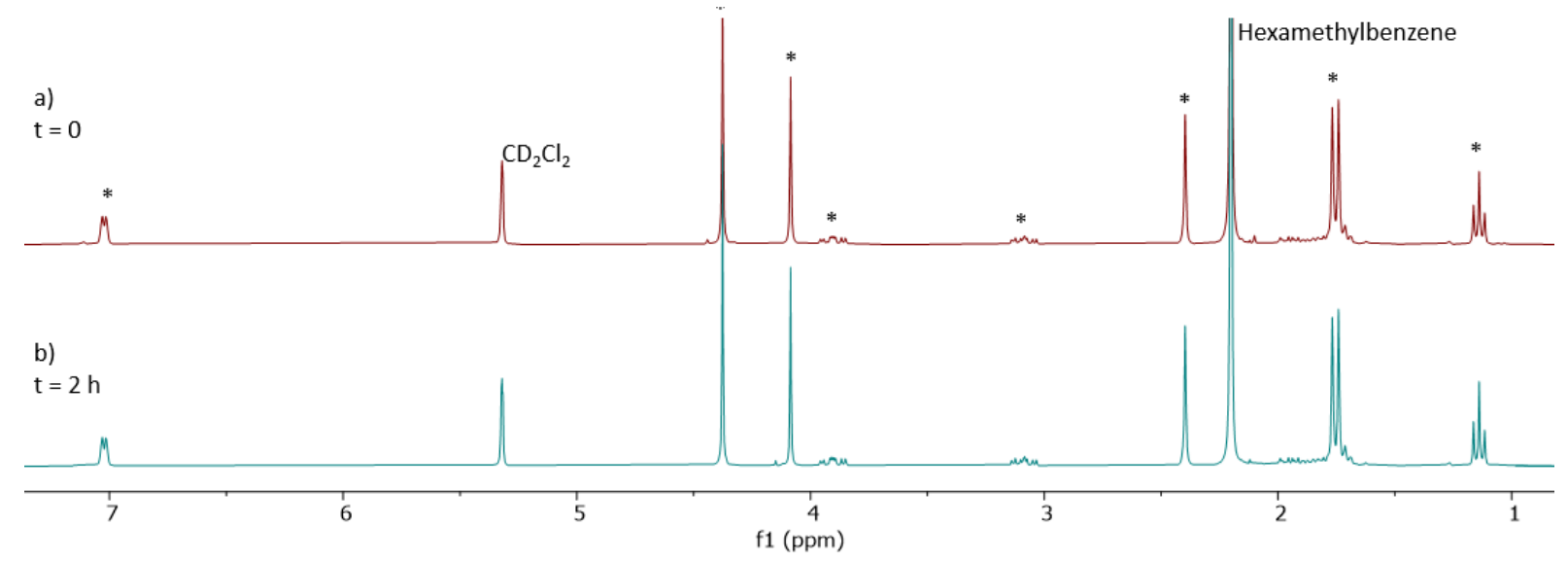

Figure S63. ${ }^{~} \mathrm{H}$ NMR spectra (300 MHz) of complex $\mathbf{5 f}(2.7 \mathrm{mg}, 5 \mu \mathrm{mol}$, indicated with $*)$ and hexamethylbenzene $(0.8 \mathrm{mg}, 25 \mu \mathrm{mol})$ in $\mathrm{CD}_{2} \mathrm{Cl}_{2}(0.5 \mathrm{~mL})$, a) start, b) $2 \mathrm{~h}$ at $60{ }^{\circ} \mathrm{C}$. 

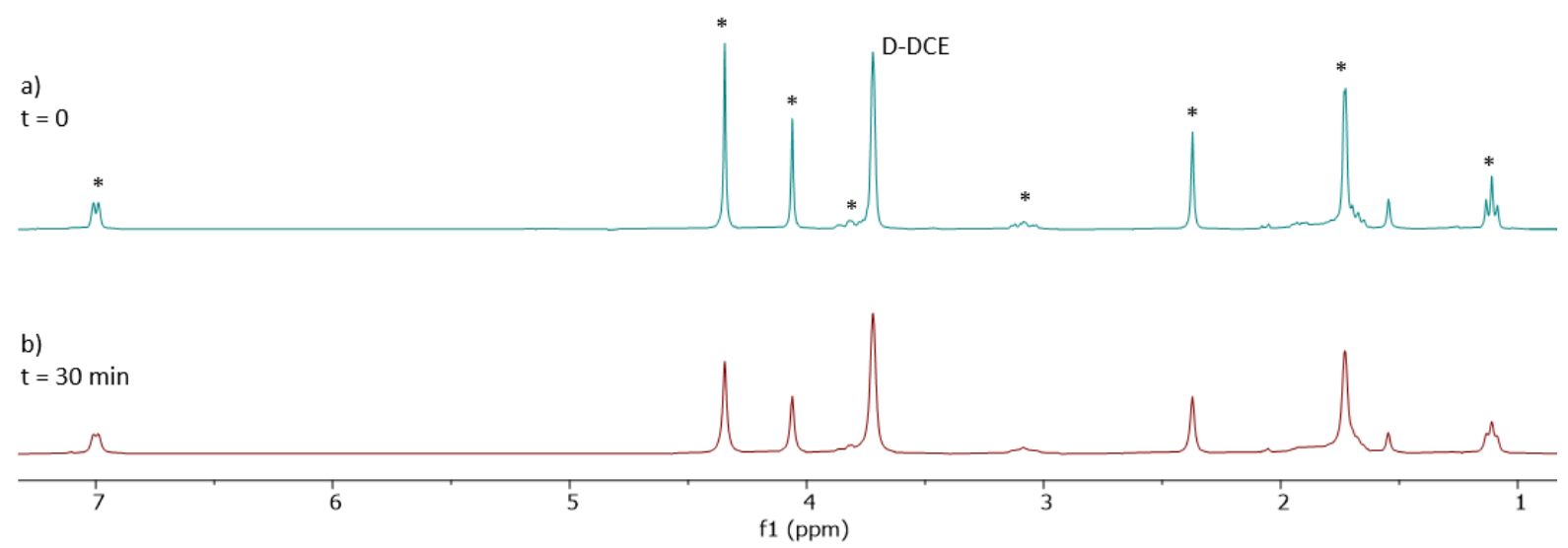

Figure S64. ${ }^{l} \mathrm{H} N M R$ spectra of (300 MHz) complex $\mathbf{5 f}(2.7 \mathrm{mg}, 5 \mu \mathrm{mol}$, indicated with *) and hexamethylbenzene $(0.8 \mathrm{mg}, 25 \mathrm{\mu mol})$ in $d-D C E(0.5 \mathrm{~mL})$, a) start, b) $30 \mathrm{~min}$ at $60^{\circ} \mathrm{C}$.
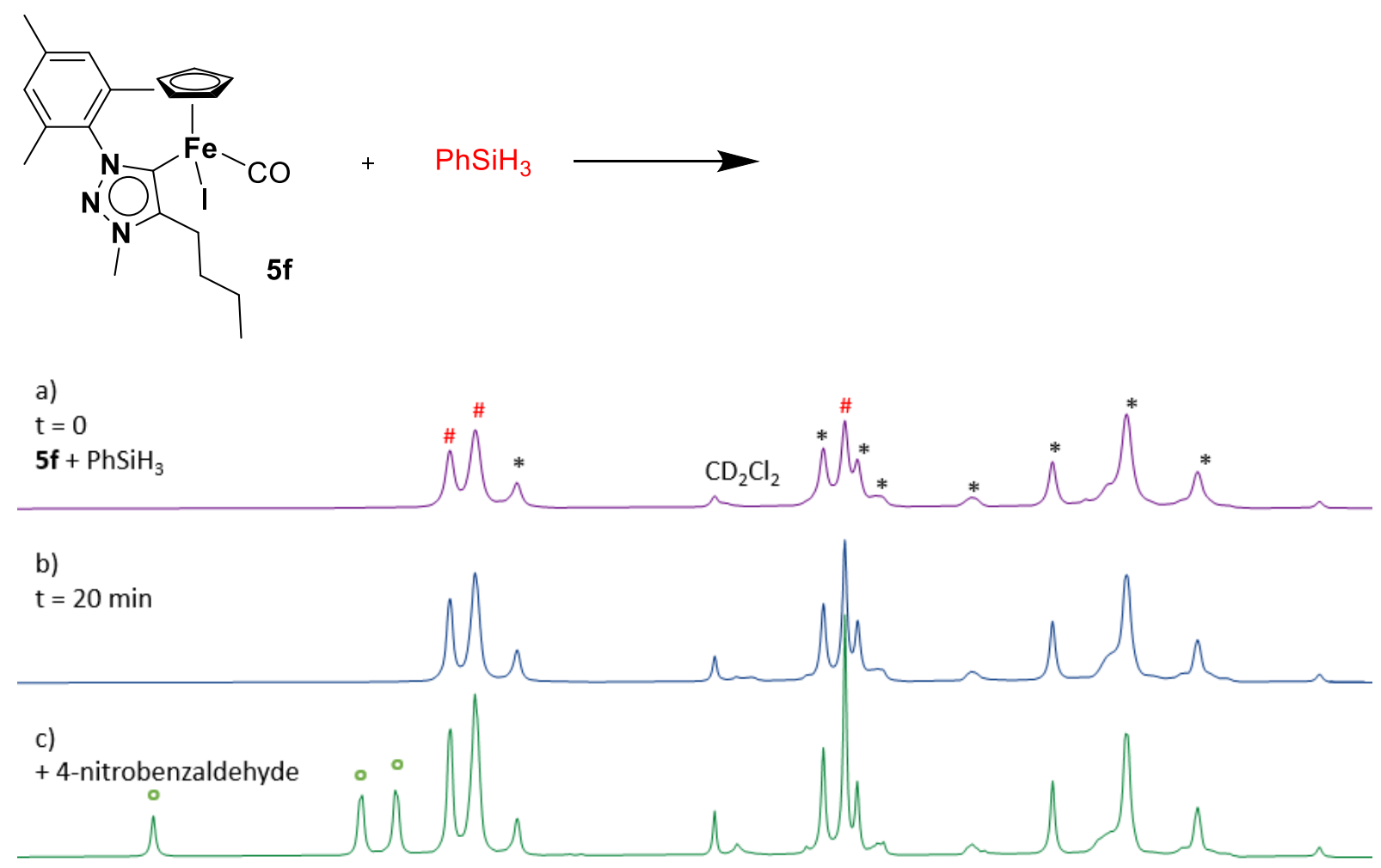

d)
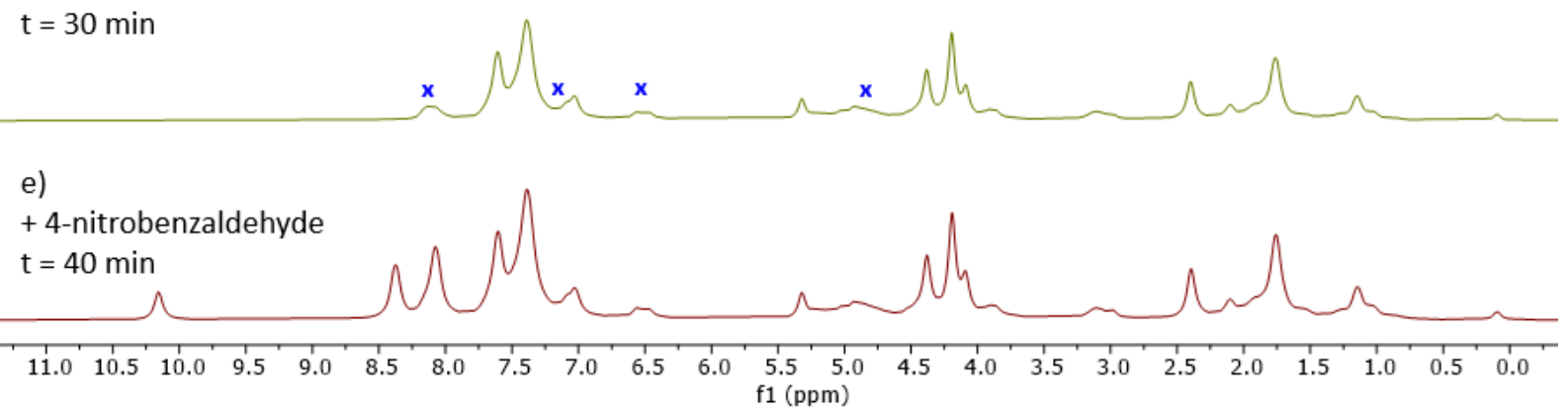

Figure S65. ${ }^{1} \mathrm{H}$ NMR spectra (300 MHz) of complex $5 \mathrm{f}\left(8 \mathrm{mg}, 15 \mu \mathrm{mol}\right.$, indicated with *) and $\mathrm{PhSiH}_{3}$ $\left(45 \mu \mathrm{mol}\right.$, indicated with \#) in $\mathrm{CD}_{2} \mathrm{Cl}_{2}(0.5 \mathrm{~mL})$, a) start, b) $20 \mathrm{~min}$ at $60{ }^{\circ} \mathrm{C}$, c) added 4nitrobenzaldehyde (2 eq., indicated with o), d) heated mixture c at $60^{\circ} \mathrm{C}$ for $10 \mathrm{~min}$, new signals indicated with $\mathbf{x}, e)$ added additional 4-nitrobenzaldehyde (2 eq.) and heated $10 \mathrm{~min}$. 


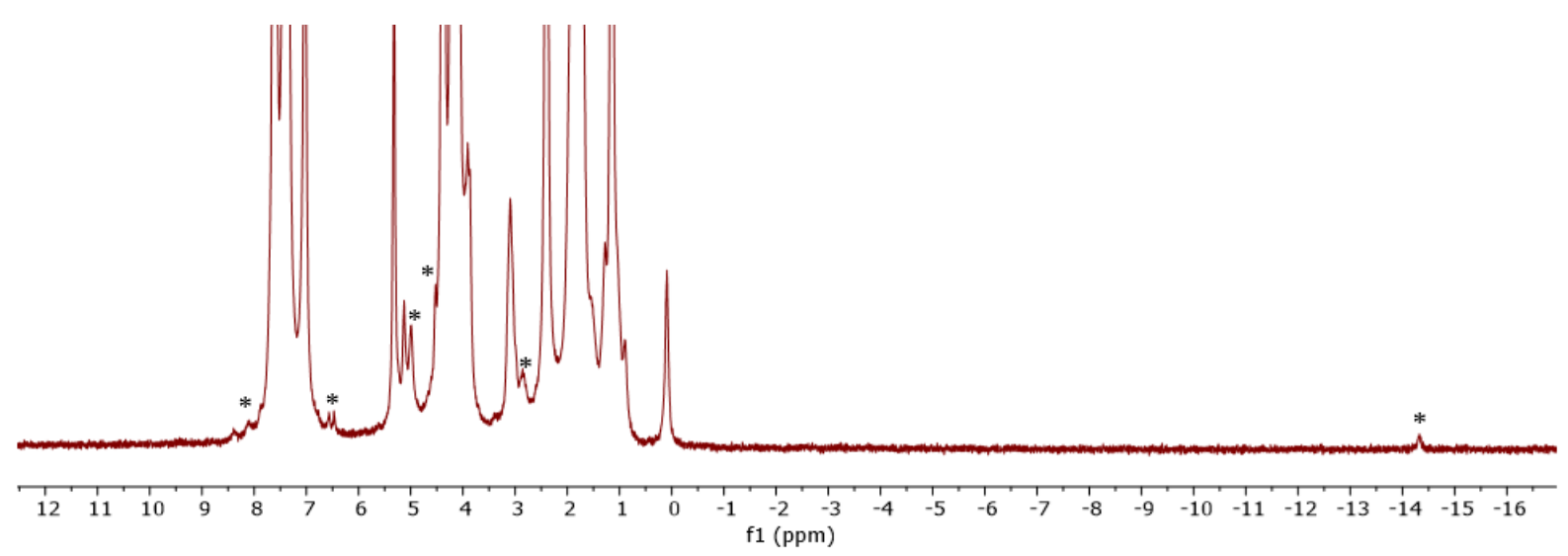

Figure S66. ${ }^{l} \mathrm{H}$ NMR spectra (300 MHz) of complex $\mathbf{5 f}(8 \mathrm{mg}, 15 \mu \mathrm{mol})$ and $\mathrm{PhSiH}_{3}(45 \mu \mathrm{mol}$, ) in $\mathrm{CD}_{2} \mathrm{Cl}_{2}(0.5 \mathrm{~mL})$, after 20 minutes at $60^{\circ} \mathrm{C}$, new signals indicated with *.

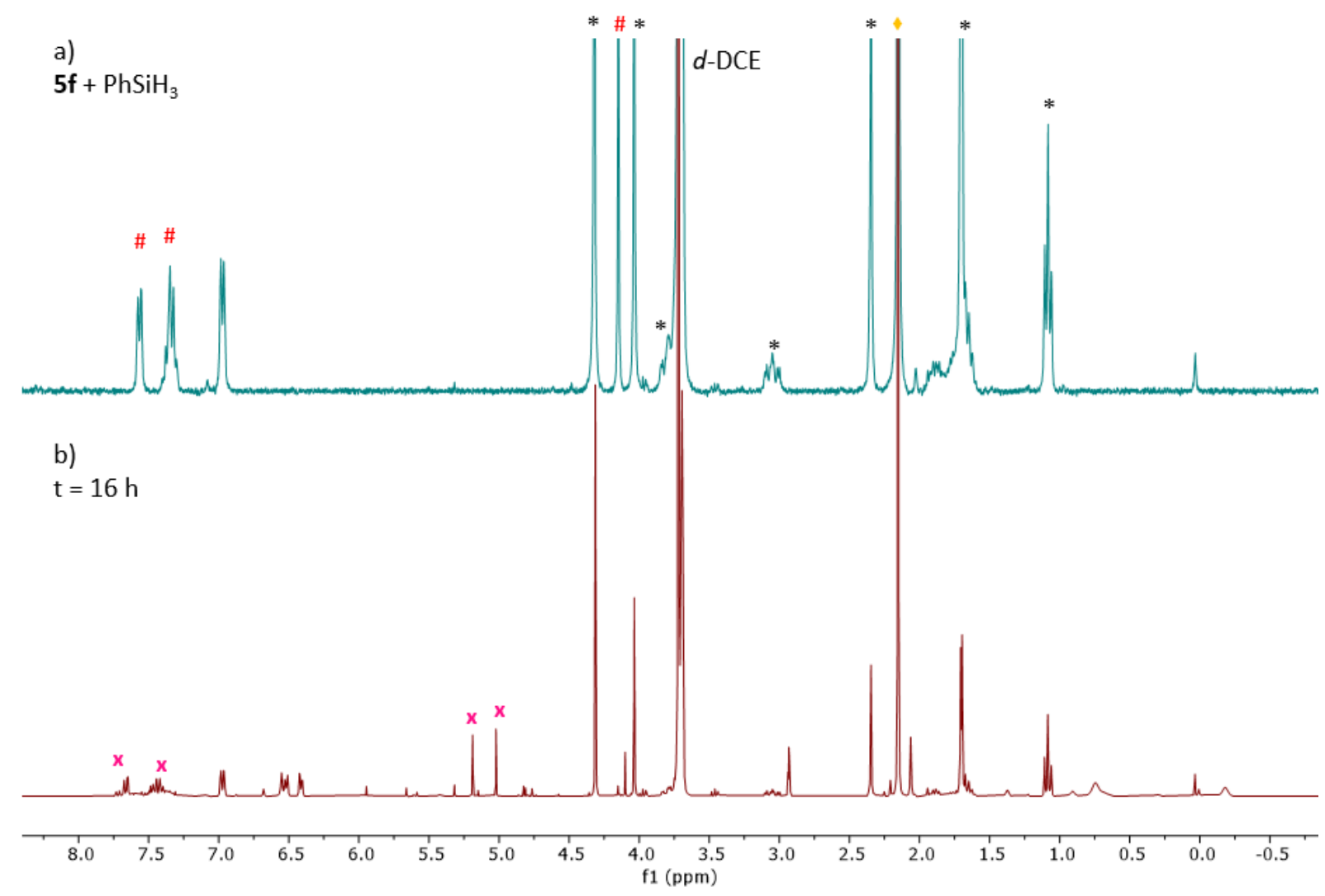

Figure S67. ${ }^{1} \mathrm{H}$ NMR spectra (300 MHz) of a) complex $\mathbf{5 f}(5 \mu \mathrm{mol}, *)$ and $\mathrm{PhSiH}_{3}(0.75 \mathrm{eq}$., \#) in 1,2dichloroethane- $d_{4}(0.5 \mathrm{~mL})$ with $\mathrm{C}_{6} \mathrm{Me}_{6}(5 \mu \mathrm{mol}, \forall)$ as internal standard at the start and b) after $16 \mathrm{~h}$ at $60^{\circ} \mathrm{C}$ and suggested products from reshuffling of $\mathrm{PhSiH}_{3}(\mathrm{x})$. 


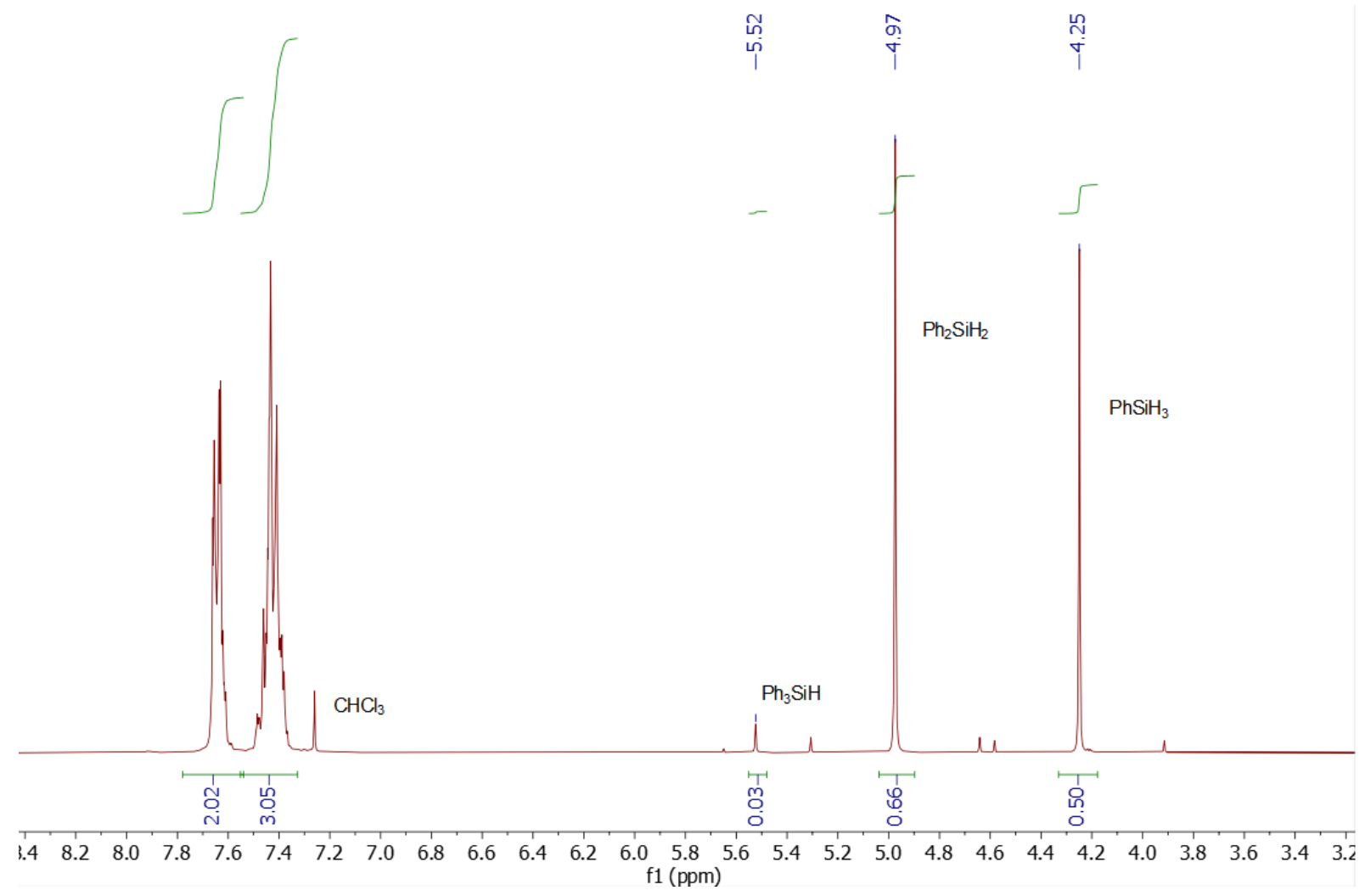

Figure S68. ${ }^{l} \mathrm{H}$ NMR spectrum (300 MHz) for $\mathrm{PhSiH}_{3}, \mathrm{Ph}_{2} \mathrm{SiH}_{2}$ and $\mathrm{PhSiH}_{3}$.

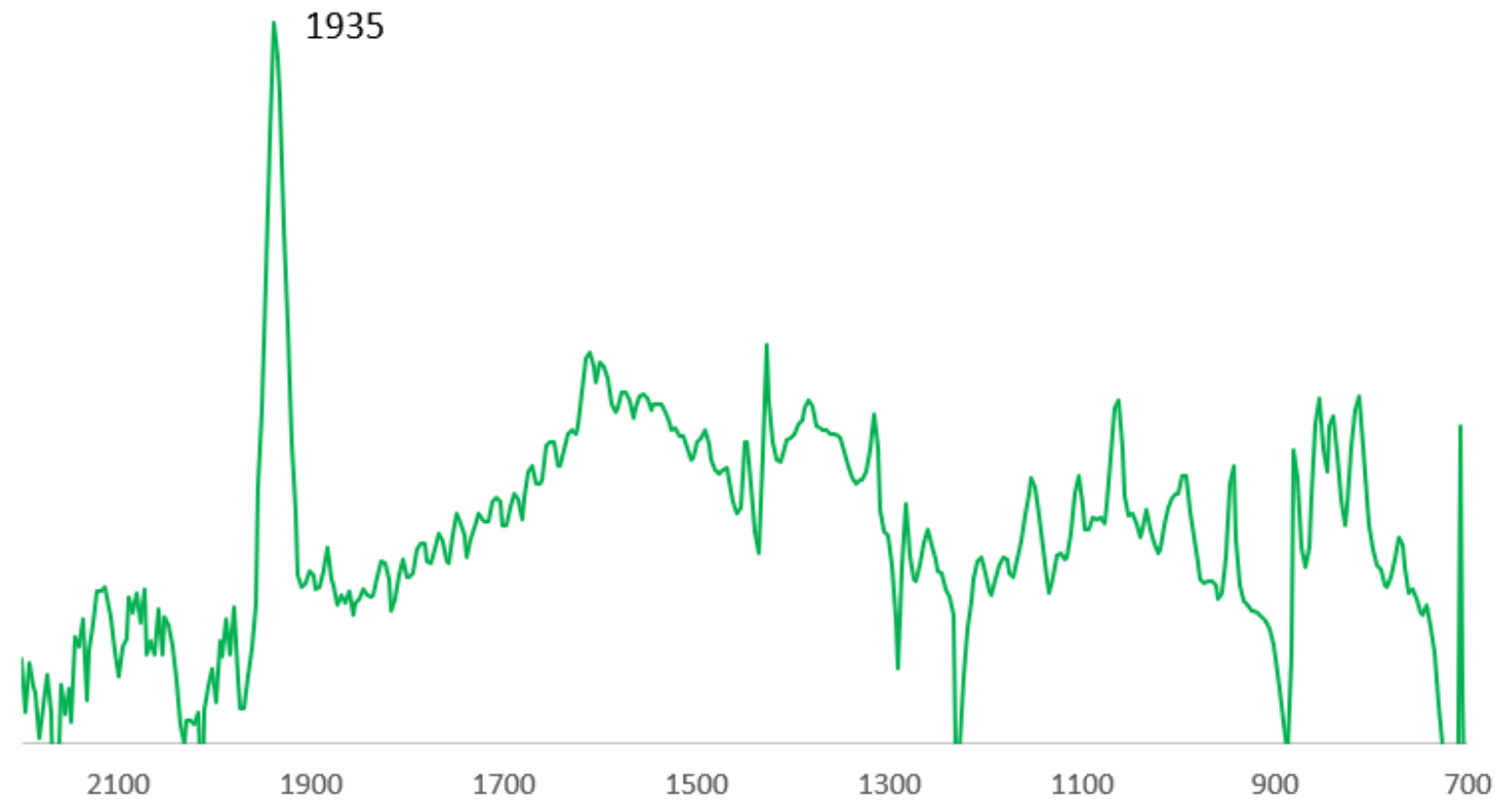

Figure S69. IR spectra of complex 5 f in 1,2-dichloroethane at $60{ }^{\circ} \mathrm{C}$, solvent signals removed by solvent subtraction. 


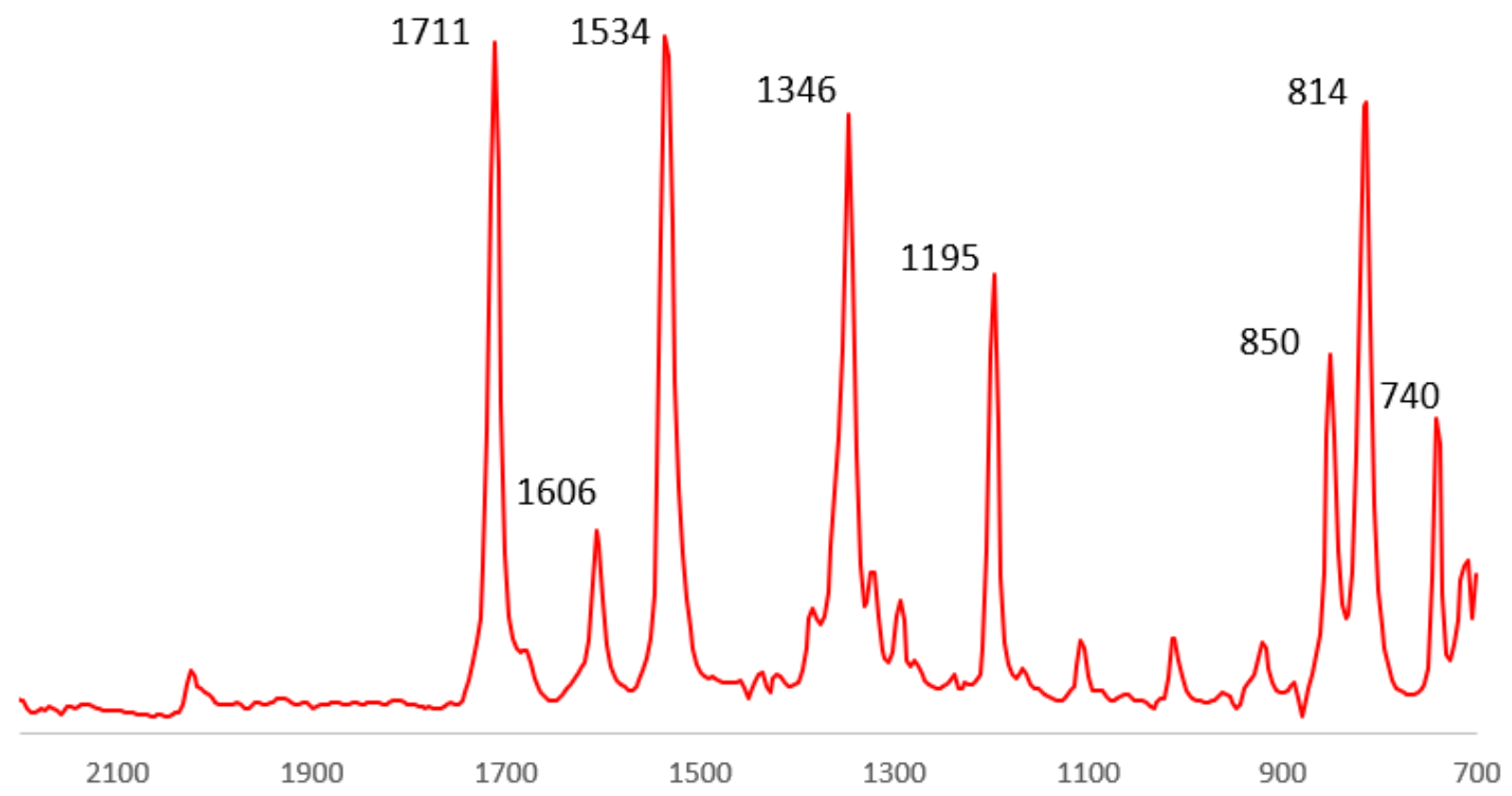

Figure S70. IR spectra of 4-benzaldehyde in 1,2-dichloroethane at $60^{\circ} \mathrm{C}$, solvent signals removed by solvent subtraction.

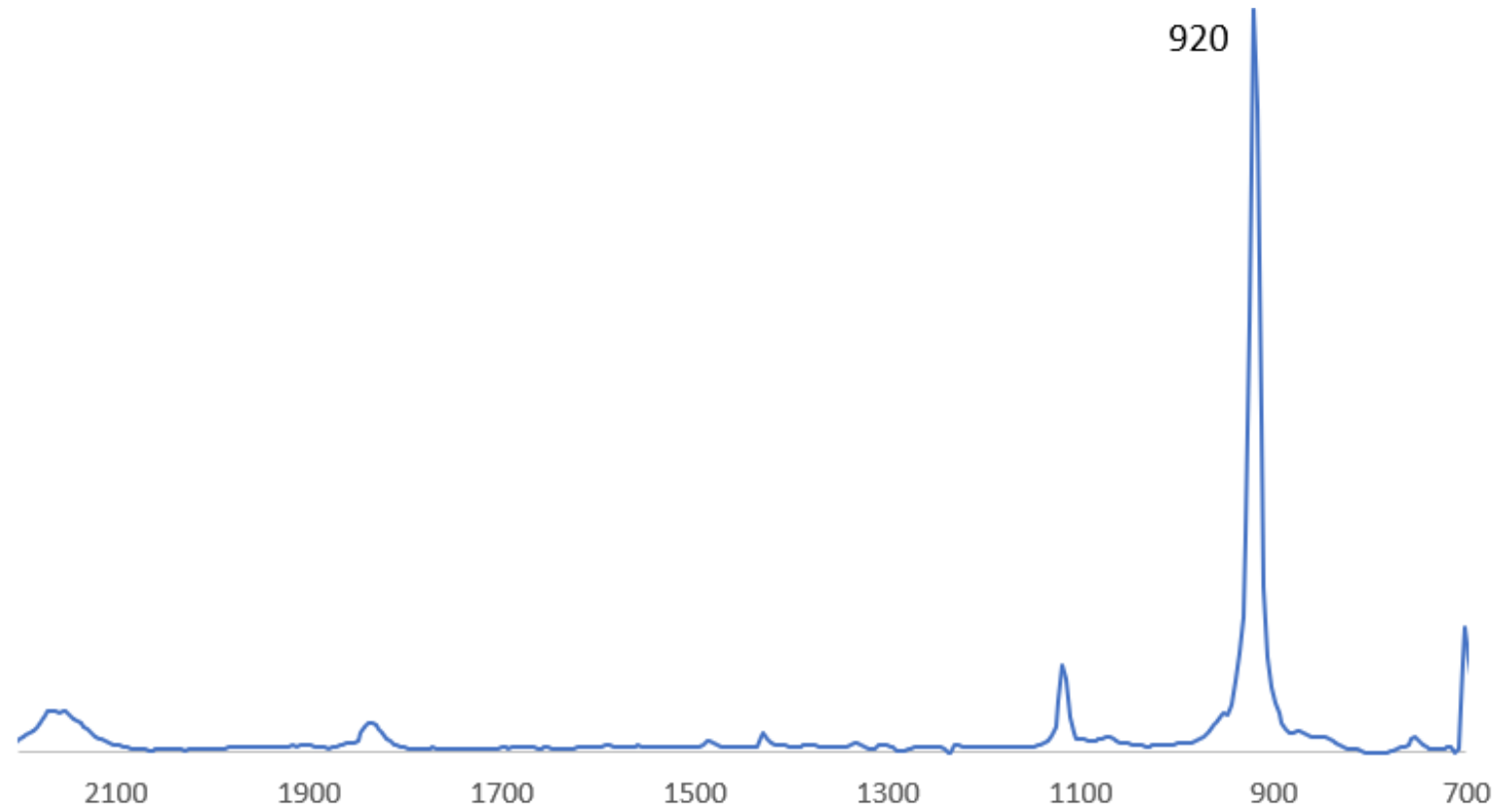

Figure S71. IR spectra of $\mathrm{PhSiH}_{3}$ in 1,2-dichloroethane at $60^{\circ} \mathrm{C}$, solvent signals removed by solvent subtraction. 


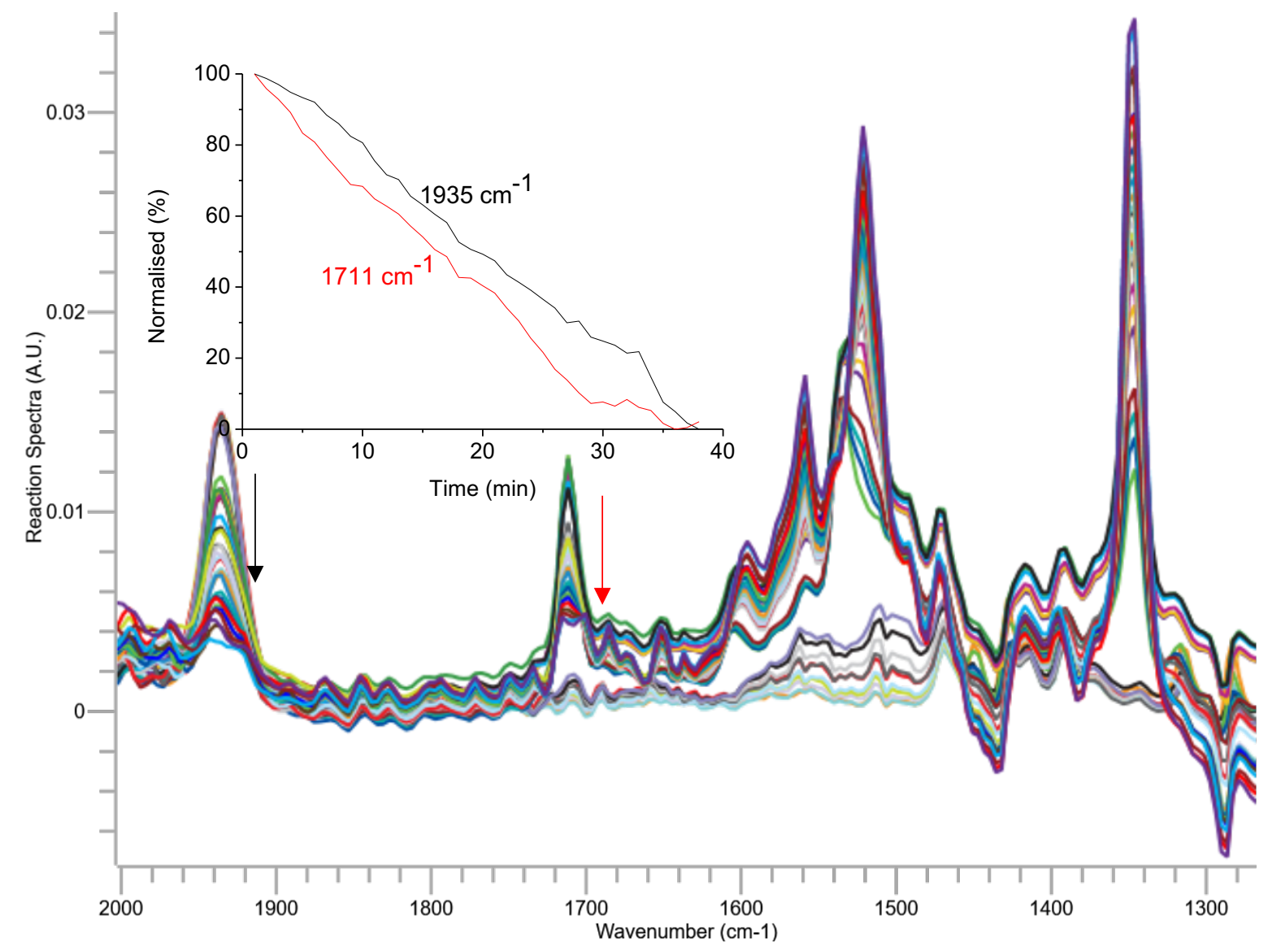

Figure $\mathbf{S 7 2 .}$ In situ FT-IR spectra recorded during the reaction between 4-nitrobenzaldehyde (10 $\mu \mathrm{mol})$ and complex $5 \mathbf{f}(10 \mu \mathrm{mol})$ in 1,2-dichloroethane $(1 \mathrm{~mL})$ at $60{ }^{\circ} \mathrm{C}$. The decrease of the intensities of the stretching frequencies is indicated with a black arrow $\left(1935 \mathrm{~cm}^{-1}\right)$ and a red arrow $\left(1711 \mathrm{~cm}^{-1}\right)$. The normalised heights of the CO stretching (black) and aldehyde stretching (red) are plotted over time.

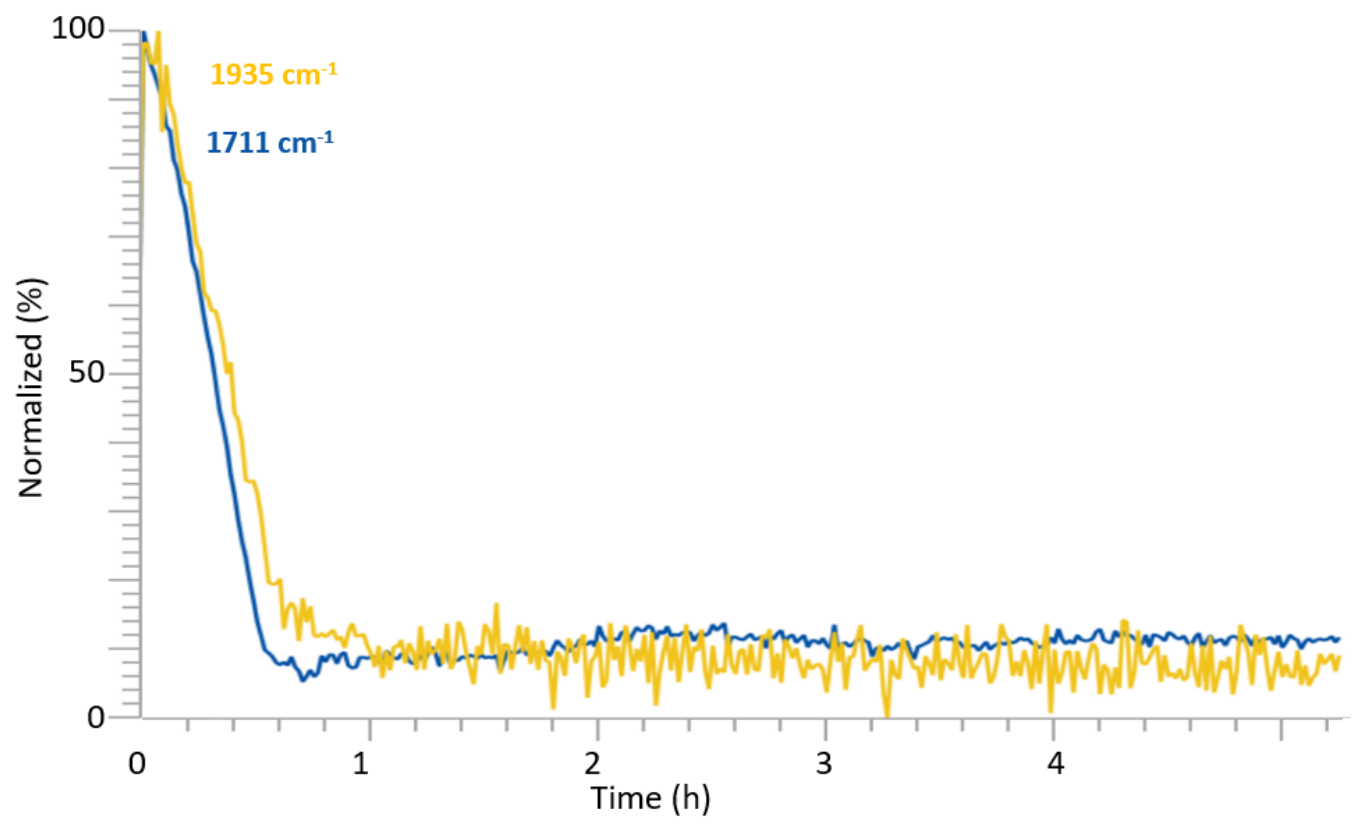

Figure S73. Conversion profile of the reaction between catalyst $\mathbf{5 f}(5 \mu \mathrm{mol}$, yellow) and 4nitrobenzaldehyde (5 $\mu \mathrm{mol}$, blue), immediately starts decreasing (IR) Measurement stopped after $5 \mathrm{~h}$. 


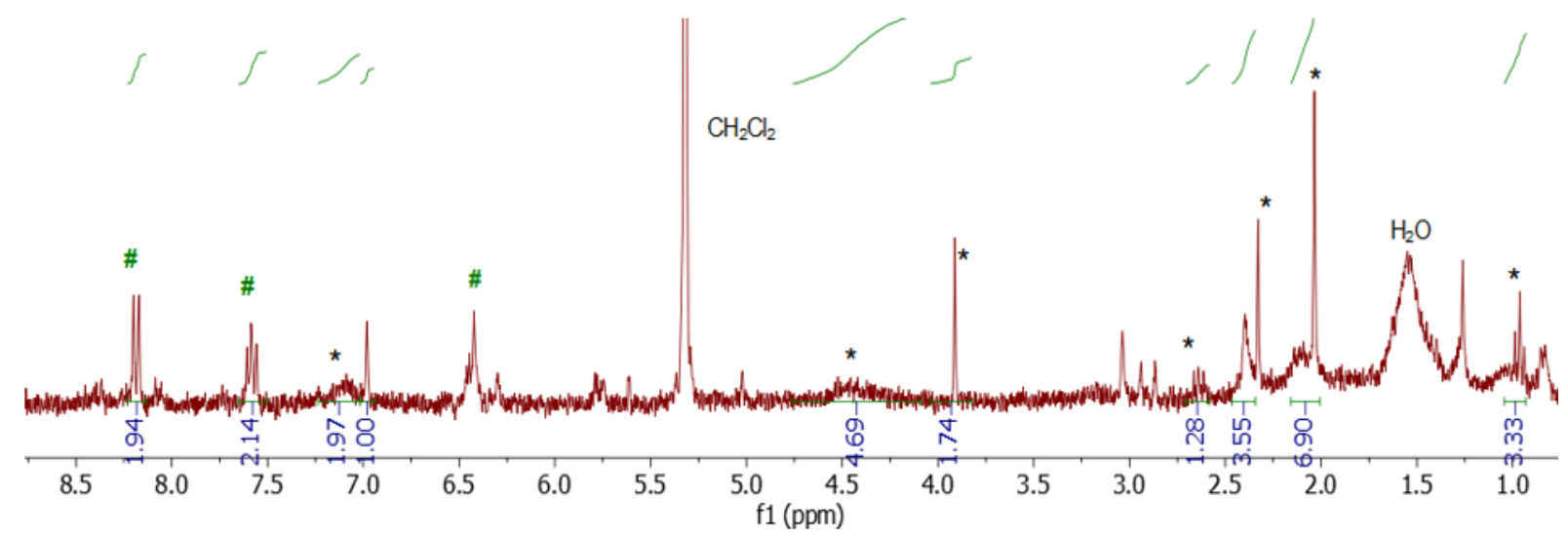

Figure $\mathbf{S 7 4 .}{ }^{1} \mathrm{H}$ NMR spectra $(300 \mathrm{MHz})$ of reaction mixture of stoichiometric amounts of complex $\mathbf{5} \boldsymbol{f}$ $(5 \mu \mathrm{mol})$ and 4-nitrobenzaldehyde (1 eq.) in $\mathrm{CD}_{2} \mathrm{Cl}_{2}(1 \mathrm{~mL})$ after filtration and evaporation of solvents after $60 \mathrm{~min}$. Proposed nitrobenzaldehyde product (\#) and free ligand signals, most likely decomposition due to work-up (*).

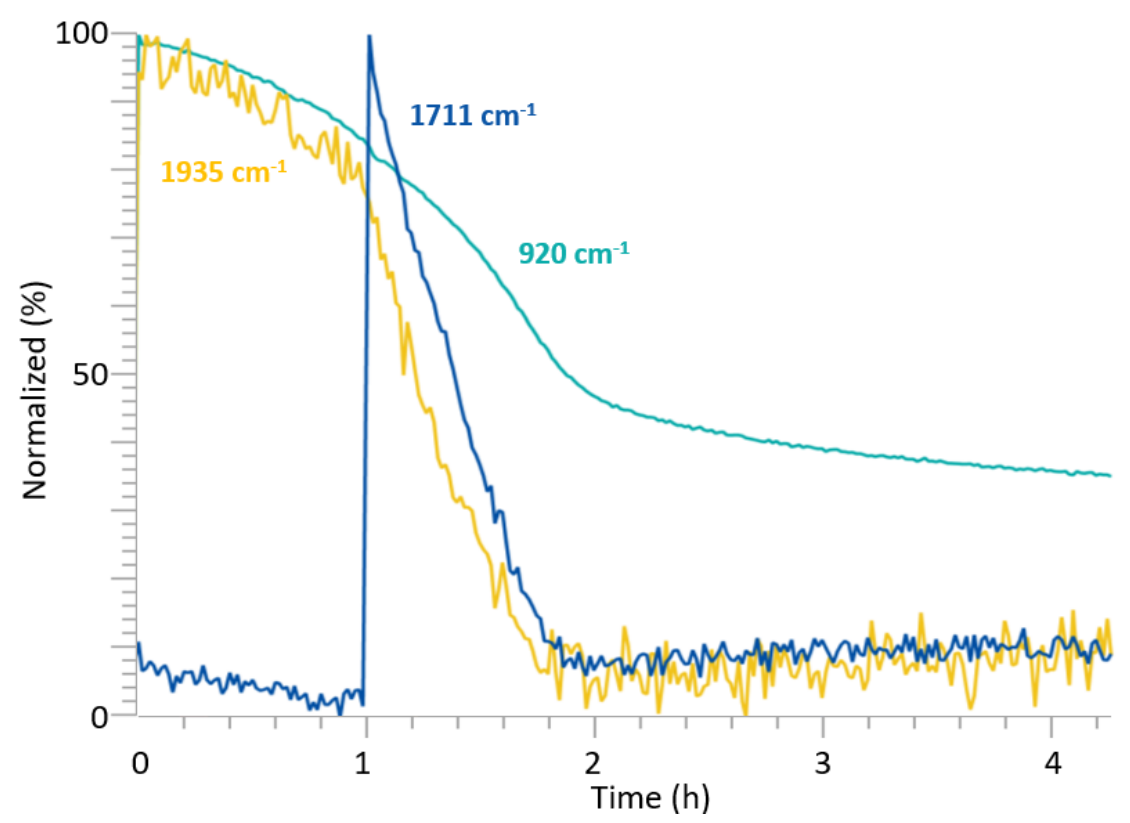

Figure S75. Conversion profile of the reaction between catalyst $\mathbf{5 f}\left(5 \mu \mathrm{mol}\right.$, yellow) and $\mathrm{PhSiH}_{3}$

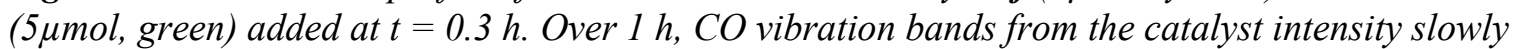
decreased. Addition of 4-nitrobenzaldehyde $(5 \mu \mathrm{mol}$, blue) at $t=1.3 \mathrm{~h}$ induced a faster decrease of both catalyst and substrate intensities. Reaction stopped at $4.5 \mathrm{~h}$. 


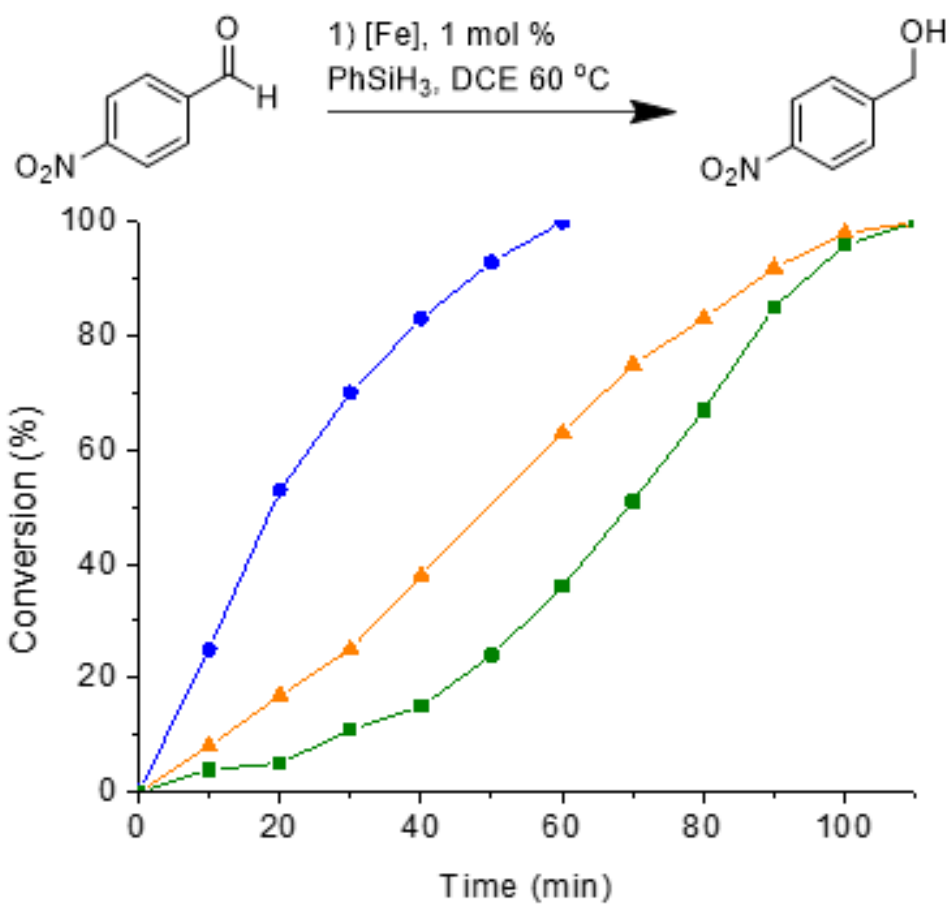

Figure S76. Time-conversion profiles for the hydrosilylation of of 4-nitrobenzaldehyde catalysed by complex $\mathbf{6} \boldsymbol{b}$. Green squares representing standard catalytic conditions as in Table S7. Orange triangles represent conversion after pre-stirring $\boldsymbol{6} \boldsymbol{b}$ and $\mathrm{PhSiH}_{3}$ under catalytic conditions for $1 \mathrm{~h}$ before addition of 4-nitrobenzaldehyde. Blue circles represent conversion after pre-stirring $\boldsymbol{6} \boldsymbol{b}$ and 4-nitrobenzaldehyde under catalytic conditions for $1 \mathrm{~h}$ before addition of $\mathrm{PhSiH}_{3}$. 


\section{References}

(S1) Lartey, M.; Meyer-Ilse, J.; Watkins, J. D.; Roth, E. A.; Bowser, S.; Kusuma, V. A.; Damodaran, K.; Zhou, X.; Haranczyk, M.; Albenze, E.; Luebke, D. R.; Hopkinson, D.; Kortright, J. B.; Nulwala, H. B. Branched Isomeric 1,2,3-Triazolium-Based lonic Liquids: New Insight into StructureProperty Relationships. Phys. Chem. Chem. Phys. 2015, 17, 29834-29843. https://doi.org/10.1039/C5CP04756K.

(S2) Overbergeb, C. G.; Salamone, J. C.; Yaboslavsky, S. Nuclear Magnetic Resonance Spectrum of the Methiodide of 1-Methylimidazole. J. Org. Chem. 1965, 30, 3580. https://doi.org/10.1021/jo01021a517.

(S3) Kriechbaum, M.; Hölbling, J.; Stammler, H. G.; List, M.; Berger, R. J. F.; Monkowius, U. Unprecedented Large Temperature Dependence of Silver(I)-Silver(I) Distances in Some NHeterocyclic Carbene Silver(I) Complex Salts. Organometallics 2013, 32, 2876-2884. https://doi.org/10.1021/om300932r.

(S4) Schaub, T.; Radius, U. Efficient C-F and C-C Activation by a Novel N-Heterocyclic CarbeneNickel(0) Complex. Chem. - A Eur. J. 2005, 11, 5024-5030. https://doi.org/10.1002/chem.200500231.

(S5) Hans, M.; Lorkowski, J.; Demonceau, A.; Delaude, L. Efficient Synthetic Protocols for the Preparation of Common N-Heterocyclic Carbene Precursors. Beilstein J. Org. Chem. 2015, 11, 2318-2325. https://doi.org/10.3762/bjoc.11.252.

(S7) Voutchkova, A. M.; Feliz, M.; Clot, E.; Eisenstein, O.; Crabtree, R. H. Imidazolium Carboxylates as Versatile and Selective N-Heterocyclic Carbene Transfer Agents: Synthesis, Mechanism, and Applications. J. Am. Chem. Soc. 2007, 129, 12834-12846. https://doi.org/10.1021/ja0742885.

(S8) Naumann, S.; Schmidt, F. G.; Schowner, R.; Frey, W.; Buchmeiser, M. R. Polymerization of Methyl Methacrylate by Latent Pre-Catalysts Based on CO2-Protected N-Heterocyclic Carbenes. Polym. Chem. 2013, 4, 2731. https://doi.org/10.1039/c3py00073g.

(S9) Poater, A.; Cosenza, B.; Correa, A.; Giudice, S.; Ragone, F.; Scarano, V.; Cavallo, L. SambVca: A Web Application for the Calculation of the Buried Volume of $\mathrm{N}$-Heterocyclic Carbene Ligands. Eur. J. Inorg. Chem. 2009, No. 13 SPEC. ISS., 1759-1766. https://doi.org/10.1002/ejic.200801160.

(S10) Falivene, L.; Poater, A.; Petta, A.; Serra, L.; Oliva, R.; Scarano, V.; Cavallo, L. SambVca 2. A Web Tool for Analyzing Catalytic Pockets with Topographic Steric Maps. Organometallics 2016, 35, 2286-2293. https://doi.org/10.1021/acs.organomet.6b00371.

(S11) Falivene, L.; Cao, Z.; Petta, A.; Serra, L.; Poater, A.; Oliva, R.; Scarano, V.; Cavallo, L. Towards the Online Computer-Aided Design of Catalytic Pockets. Nat. Chem. 2019, 11, 872-879. https://doi.org/10.1038/s41557-019-0319-5. 